\title{
Stochastic finite elements of discretely parameterized random systems on domains with boundary uncertainty
}

\author{
A. Kundu* ${ }^{* \dagger}$, S. Adhikari and M. I. Friswell \\ Swansea University, Singleton Park, Swansea, SA2 8PP, Wales, UK
}

\begin{abstract}
SUMMARY
The problem of representing random fields describing the material and boundary properties of the physical system at discrete points of the spatial domain is studied in the context of linear stochastic finite element method. A randomly parameterized diffusion system with a set of independent identically distributed stochastic variables is considered. The discretized parametric fields are interpolated within each element with multidimensional Lagrange polynomials and integrated into the weak formulation. The proposed discretized random-field representation has been utilized to express the random fluctuations of the domain boundary with nodal position coordinates and a set of random variables. The description of the boundary perturbation has been incorporated into the weak stochastic finite element formulation using a stochastic isoparametric mapping of the random domain to a deterministic master domain. A method for obtaining the linear system of equations under the proposed mapping using generic finite element weak formulation and the stochastic spectral Galerkin framework is studied in detail. The treatment presents a unified way of handling the parametric uncertainty and random boundary fluctuations for dynamic systems. The convergence behavior of the proposed methodologies has been demonstrated with numerical examples to establish the validity of the numerical scheme. Copyright $\odot 2014$ John Wiley \& Sons, Ltd.
\end{abstract}

Received 10 October 2013; Revised 3 June 2014; Accepted 5 June 2014

KEY WORDS: random boundary; stochastic finite element analysis; probabilistic methods; stochastic problems; polynomial chaos expansion; parametric uncertainty

\section{INTRODUCTION}

Uncertainty is present in a wide range of problems tackled within the scope of computational mechanics and is incorporated into the mathematical models in the form of random physical parameters, random forcing functions, and random geometrical descriptions. This gives rise to stochastic partial differential equation (SPDE). The resolution of these SPDE and propagation of uncertainty into the system response have called for efficient numerical methods to tackle these expensive problems that range from non-intrusive efficient Monte Carlo and quasi-Monte Carlo techniques to the intrusive stochastic spectral Galerkin methods. These have been aptly summed up in the recent research articles [1-3].

The focus of the current work is on the parametric and geometrical uncertainties in computational mechanics within the framework of the stochastic finite element (FE) method. The first part is concerned with the stochastic discretization of the random field. A discrete Karhunen-Loève (KL) expansion is investigated in this study, where the principal spectral components of the covariance kernel are obtained by solving a simple eigenvalue problem of the covariance matrix constructed at the discrete set of points on the spatial domain. This can alleviate the computational complexity associated with the numerical solution of the KL expansion. The resulting discrete formulation is interpolated inside the element domain at the quadrature integration points using spatial interpolation functions.

\footnotetext{
*Correspondence to: A. Kundu, Swansea University, Singleton Park, Swansea, SA2 8PP, Wales, UK.

†E-mail: a.kundu.577613@ @swansea.ac.uk
} 
Accounting for surface roughness in the mathematical models is an important consideration for systems where random perturbations of domain topologies can have considerable impact on the accuracy of the numerical results. This phenomenon can be widely observed in nano-scale designs, high-speed-flow problems, aerodynamic systems, thermal systems with boundary flux, and corrosion, to mention a few. The boundary fluctuations have been tackled with a number of methods ranging from parameterization of surface inhomogeneities to using fractals. The framework for the solution of problems on random domains and their error bounds has been presented for Neumann [4] and Dirichlet [5] boundary value problems. Other studies involve natural convection with sparse-grid collocation technique [6], flow problems with multi-element collocation technique [7], transport in rough walled tubes [8], acoustic scattering from rough surfaces [9] inspired from the transformation to obtain boundary perturbations [10]. Also, random domains have been represented with fractals and studied with the Monte Carlo simulation technique [11], which turned out to be computationally expensive. A novel framework for tackling the random boundary problems with stochastic mapping has been studied in [12], where the concept of a boundary-conforming coordinate system [13] has been used to represent a parameterized boundary fluctuation. However, a generic methodology for tackling the problem of geometric uncertainty within the scope of the stochastic spectral Galerkin formulation is still an open area of research and has been investigated in the present work.

Here, we consider the boundary perturbations with a discrete random-field description, which can approximate the prescribed second-order statistics associated with this random variation. Following this, a stochastic isoparametric mapping would be utilized to establish a generic framework within which the random geometry description can be incorporated into the stochastic weak formulation. Using this, it would be possible to obtain an accurate estimation of the stochastic response statistics associated with the random boundary. The stochastic Galerkin method employed for approximating the solution of an SPDE provides an accurate estimation of the second-moment properties of the system solution. But it requires high-order polynomial functions to capture the higher-order moments and the entire probability distribution. Hence, solving the SPDE with the stochastic Galerkin method in this paper refers to estimating the second-order statistics of the response.

The paper is organized as follows. In Section 2, we give a brief overview of the random-field representation with a set of random variables using various series expansion techniques. Section 3 presents the discussion on the discrete spectral decomposition of the covariance function. Section 4 details the methodology to incorporate the discrete random-field description into the weak form of the SPDE for a steady-state diffusion problem within the stochastic spectral Galerkin framework. Then Section 5 details the stochastic isoparametric mapping used to integrate the discrete randomfield description with the stochastic spectral Galerkin method. Section 5 presents the description of the boundary roughness and the stochastic isoparametric mapping technique from the random domain to the deterministic parent domain. Following this, Section 7 demonstrates the proposed techniques for the spectral decomposition of the random field with numerical examples and error analysis. Then we include some numerical examples to demonstrate the random boundary problem with a transient stochastic diffusion system. Section 8 lists the principal conclusions that can be drawn from this work and the directions of future research.

\section{FINITE-DIMENSIONAL RANDOM-FIELD REPRESENTATION: OVERVIEW}

\subsection{Probabilistic description of input uncertainty}

We define a spatially correlated random field $\alpha$ on a compact bounded domain $\mathscr{D} \subset \mathbb{R}^{d}$ with probability support $(\Theta, \mathscr{F}, P)$; thus, $\alpha: \mathscr{D} \times \Theta \rightarrow \mathbb{R}$. Here, $\theta \in \Theta$ is a sample point from the sampling space $\Theta, \mathscr{F}$ is the complete Borel $\sigma$-algebra over the subsets of $\Theta$, and $P$ is the probability measure. The uncertainty characterization of the random field can be performed using 'probabilistic characterization', which defines indexed finite-dimensional joint distribution functions as $[14,15]$

$$
F_{r_{1}, \ldots, r_{n}}\left(\xi_{1}, \ldots, \xi_{n}\right)=P\left\{\alpha\left(\mathbf{r}_{i}\right) \leqslant \xi_{i}, \forall i=1, \ldots, n\right\}, \quad \mathbf{r}_{i} \in \mathscr{D}, \quad \xi_{i} \in \mathbb{R} \quad \forall i=1, \ldots, n
$$


such that the random field is defined as a set of random variables $\alpha(\mathbf{r}):=\alpha(\mathbf{r}, \cdot): \Theta \rightarrow \mathbb{R}$, where $\Theta$ is characterized by $F_{r_{1}, \ldots, r_{n}}\left(\xi_{1}, \ldots, \xi_{n}\right)$. $\Theta$ can be constructed from the finite-dimensional distribution functions under weak-consistency conditions [15]. Alternatively, the random field is defined as a random variable whose elementary events $\theta$ in the bounded region $\mathscr{D} \subset \mathbb{R}^{d}$ are realized as

$$
\alpha(\cdot, \theta): \mathscr{D} \rightarrow \mathbb{R}, \quad \text { where } \quad \theta(\mathbf{r}) \equiv \alpha(\mathbf{r}, \theta) ; \quad \mathbf{r} \in \mathscr{D}, \theta \in \Theta, \text { such that } \Theta \subset\{\theta \mid \theta: \mathscr{D} \rightarrow \mathbb{R}\}
$$

The preceding definition necessitates specifying a probability measure $P_{\alpha}$ on the function space $\Theta$ with a finite-dimensional probability distribution. However, for most engineering applications, it is not practical to specify the random field with all its finite-dimensional distributions or with the measures on a probability space.

Gaussian models are frequently used in the modeling of random fields because the central limit theorem ensures their natural occurrence and they give the maximum entropy model if only secondorder information is available. Given any second-order statistics, it is possible to find a Gaussian random field $\alpha(\mathbf{r}, \theta)$ that satisfies them. Non-Gaussian random field $\alpha(\mathbf{r}, \theta)$ with a distribution function $F_{\alpha}$ can be defined with a non-linear transformation

$$
\alpha(\mathbf{r}, \theta)=\eta(\mathbf{r}, \xi(\mathbf{r}, \theta)):=F_{\alpha(\mathbf{r})}^{-1} \circ \operatorname{erf}(\xi(\mathbf{r}, \theta))
$$

where $\alpha(\mathbf{r}, \theta)$ has a marginal distribution $F_{\alpha}(\mathbf{r})$ at any point $\mathbf{r} \in \mathscr{D}$. Hence, given the secondorder statistics of a non-Gaussian random field $\alpha$, it is necessary to select $\eta$ and the covariance of the normal random variables $C_{\xi}\left(\mathbf{r}_{1}, \mathbf{r}_{2}\right)$. This is often a non-trivial exercise, and arbitrary choices of marginal distribution and/or the target covariance may lead to inconsistencies. Some analytical expressions for various marginal distributions can be found in [16, 17]. Another approach of representing random fields with finite-dimensional distribution functions is to adopt a series expansion method using the spectral properties of the covariance kernel of the second-order random field, which is discussed in the following section.

\subsection{Series expansion techniques for finite-dimensional representation of random field}

Most works in stochastic FEs concerned with the solution of randomly parameterized systems generally assume a mathematically tractable model for the random field, which might not always be experimentally justifiable. For these applications, the random fields are chosen to obey certain physically meaningful regularity assumptions, such as mean-square continuity, homogeneity, and isotropy $[18,19]$. Given an admissible covariance description of the input random field, the parameter can be written in a variable separable form as

$$
a(\mathbf{r}, \theta)=\sum_{i} \varphi_{i}(\mathbf{r}) \kappa(\theta)=\varphi(\mathbf{r}) \mathscr{K}(\theta)
$$

where $\mathscr{K}(\theta)=\left\{\kappa_{1}(\theta), \ldots, \kappa_{m}(\theta)\right\}^{T}$ is a random function vector weighted by the spatial shape functions $\varphi(\mathbf{r})=\left\{\varphi_{1}(\mathbf{r}), \ldots, \varphi_{m}(\mathbf{r})\right\}$.

The spatial shape functions can be chosen using various methods such as the interpolation method [20], the mid-point method [21], the expansion optimal linear estimation method [21], and the spatial average method [22] to name a few. Another important class of method is the spectral decomposition of the random field. This relies on discretizing the random field with a finite number of spectral components of the covariance kernel associated with the random process. We discuss this last approach in some detail here.

Let $C_{a}: \mathscr{D} \times \mathscr{D} \rightarrow \mathbb{R}$ be a kernel function that admits to the following decomposition

$$
\int_{\mathscr{D}} C_{a}\left(\mathbf{r}_{1}, \mathbf{r}_{2}\right) \varphi_{j}\left(\mathbf{r}_{1}\right) \mathrm{d} \mathbf{r}_{1}=v_{j} \varphi_{j}\left(\mathbf{r}_{2}\right), \quad \forall j=1,2, \cdots
$$


The preceding expression is a homogeneous Fredholm integral equation of the second kind. The KL expansion is such a spectral representation of the random field using orthogonal eigen-components of the covariance kernel. Hence, for the random parameter $a(\mathbf{r}, \theta)$, the truncated spectral representation takes the form of

$$
a(\mathbf{r}, \theta)=a_{0}(\mathbf{r})+\sum_{i=1}^{m} \sqrt{v_{i}} \xi_{i}(\theta) \varphi_{i}(\mathbf{r})
$$

where $a_{0}(\mathbf{r})=\mathbb{E}[a(\mathbf{r}, \theta)]$ is the mean of the stochastic parameter and $\xi_{i}(\theta)$ are the mutually uncorrelated random variables with zero mean and unit variance $\left(\mathbb{E}\left[\xi_{i}(\theta)\right]=0 \& \mathbb{E}\left[\xi_{i}^{2}(\theta)\right]=\right.$ $1, \forall i=1, \ldots, m)$. In addition, $v_{i}$ and $\varphi_{i}(\mathbf{r})$ are eigenvalues and eigenfunctions satisfying the integral equation in (5). Now, for Gaussian random fields, $\xi_{i}$ are uncorrelated Gaussian random variables by virtue of the property of Gaussian variables. However, the Gaussian random-field model is not applicable for strictly positive quantities arising in many practical problems.

When $a(\mathbf{r}, \theta)$ is a general non-Gaussian random field, it can be expressed in a mean-square convergent series with stochastic chaos expansion as $a(\mathbf{r}, \theta)=a_{0}(\mathbf{r})+\sum_{i=1}^{m} \mathscr{H}_{i}(\xi(\theta)) a_{i}(\mathbf{r})$ using the Wiener-Askey chaos expansion scheme [23], where $\mathscr{H}_{i}(\xi(\theta))$ are orthogonal polynomials spanning the stochastic Hilbert space. $\mathscr{H}_{i}(\xi(\theta))$ are functions of the $M$ basic random variables $\xi(\theta)=\left\{\xi_{1}, \ldots, \xi_{M}\right\}$. Thus, the stochastic problem can be equivalently formulated on the finitedimensional probability space $\left(\Theta^{(M)}, \mathscr{F}^{(M)}, P^{(M)}\right)$, where $\Theta^{(M)}=\operatorname{Range}(\xi)$ is a subset of $\mathbb{R}^{M}$, $\mathscr{F}^{(M)}$ is the associated Borel $\sigma$-algebra, and $P^{(M)}$ is the image probability measure. It might be mentioned that while KL expansion has been used in this study, it gives a mean-square convergence of non-Gaussian random fields with prescribed second-moment properties. When such a description of the input random field is not available, the various non-Gaussian random-field approximation theories [17] have to be utilized.

In the following section, we propose an approach of expressing the random field at a finite set of points on physical domain using a discrete version of the KL expansion for the representation of the random field. This approach is particularly useful for the representation of the random field on arbitrarily shaped domains and for any complicated covariance function when analytical solutions of the KL eigenmodes are not available.

\section{NUMERICAL METHODS FOR SPECTRAL DECOMPOSITION OF THE COVARIANCE FUNCTION}

The KL expansion presented in Equation (6) represents the random parameter with a finite number of random variables weighted by the spatial eigen-basis, which are evaluated from the decomposition of the covariance kernel. However, exact solutions of the Fredholm integral equations of the second type, Equation (5), are available for a few specific types of covariance kernels and a few special geometries. A number of solutions of the decomposition of the covariance kernel for one-dimensional domains have been given in [24, 25]. However, analytical methods of decomposition of the kernel function are not available for complicated generalized spatial domains, and numerical methods have to be employed to tackle these problems, such as in [26, 27]. Here, we present a solution methodology that solves the spectral decomposition of the covariance kernel at a discrete set of points on the physical domain.

\subsection{Discrete Karhunen-Lò̀ve expansion}

If we consider the various geometrical configurations shown in Figure 1, we see that there are a number of possible definitions of the correlation length associated with the covariance kernel description. For example, the 'red' lines denote the $L^{2}$ length between points $P_{1}$ and $P_{2}$, while the 'blue' lines denote a length defined along the geometry, which would be considered as an $L^{1}$ norm. The resolution of the KL expansion for such varied kinds of geometry might result in discontinuous eigenfunctions that cannot be described with closed-form analytical functions. Additionally, we should also take into account the discrete index based correlation of the various parameters (such as 


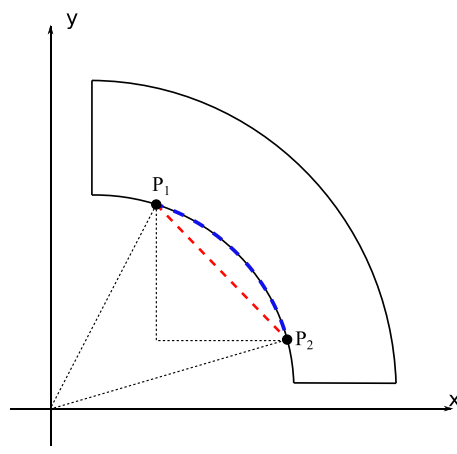

(a) Configuration 1

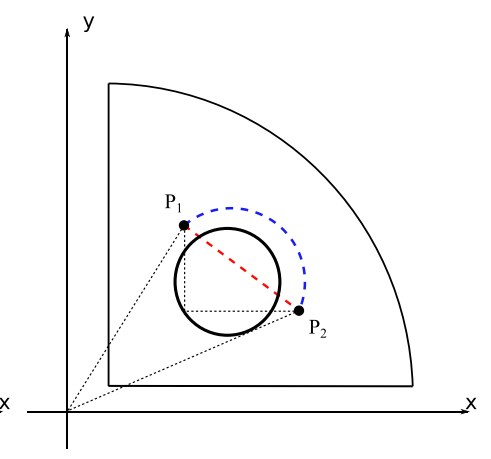

(b) Configuration 2

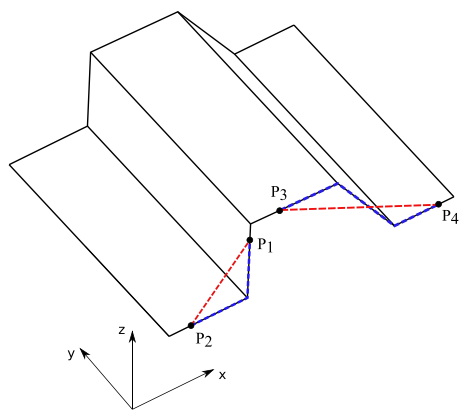

(c) Configuration 3

Figure 1. Different geometrical configurations highlighting the various possible definitions of the correlation length of the random field. (a) Configuration 1. (b) Configuration 2. (c) Configuration 3.

angles, widths, and heights of corrugation in Figure 1(c) across the length of the panel) and include them within the covariance description of the random field.

Let us define the function $\mathscr{C}_{a} \varphi$ such that

$$
\left(\mathscr{C}_{a} \varphi\right)\left(\mathbf{r}_{1}\right)=\int_{\mathscr{D}} C_{a}\left(\mathbf{r}_{1}, \mathbf{r}_{2}\right) \varphi\left(\mathbf{r}_{2}\right) d \mathbf{r}_{2}, \quad \text { where } \quad \mathbf{r}_{1}, \mathbf{r}_{2} \in \mathscr{D}
$$

It can be easily verified that $\mathscr{C}_{a}: L^{2}(\mathscr{D}) \rightarrow L^{2}(\mathscr{D})$ is a linear operator on a vector space, and hence, Equation (5) can be expressed as

$$
\mathscr{C}_{a} \varphi=v \varphi
$$

A non-trivial solution to the preceding homogeneous equation exists only for those values of $v$ that make $\left(I-v \mathscr{C}_{a}\right)$ non-invertible, where $I$ is the identity operator. Considering the fact that the solution of Equation (5) lies in some normed vector space, it is possible to represent the random field using a finite number of dominant components based on the eigen-spectrum of the kernel. This leads to a computationally feasible solution methodology.

The covariance functions $C_{a}$ commonly encountered in the study of randomly parameterized engineering systems are bounded and symmetric; hence, the associated linear operator $\mathscr{C}_{a}$ is compact and self-adjoint. Hence, the solution of the eigenvalue problem in (8) yields ordered real eigenvalues $v=\left\{v_{i}: v_{i} \geqslant v_{i+1} \forall i\right.$ and $\left.\left\|C_{a}\right\|_{L^{2}(\mathscr{D} \times \mathscr{D})}^{2}=\sum_{i=1}^{\infty} v_{i}^{2}\right\}$ and mutually orthogonal eigenfunctions in $\mathscr{D} \subset L^{2}\left(\mathbb{R}^{d}\right)$. Thus, the error in approximating the covariance function with a finite number $(m)$ of eigenfunctions results in an error that can be expressed as

$$
\text { error }\left\|C_{a}-C_{a}^{(m)}\right\|_{L^{2}(\mathscr{D} \times \mathscr{D})}=\sum_{i=m+1}^{\infty} v_{i}^{2}
$$

where $C_{a}^{(m)}$ is the covariance function approximated with $m$ eigenvalues. Thus, the approximate covariance function can be expressed as (using Mercer's theorem)

$$
C_{a}^{(m)}\left(\mathbf{r}_{1}, \mathbf{r}_{2}\right)=\sum_{i=1}^{m} \nu_{i} \varphi\left(\mathbf{r}_{1}\right) \varphi\left(\mathbf{r}_{2}\right)
$$

where $C_{a}^{(m)}$ converges uniformly to $C_{a}$ as $m \rightarrow \infty$. We discretize the spatial domain on which the solution of the integral equation is sought. Commonly, the spatial domain is discretized with the FE 
points, and hence, the random parameter can be expressed on them with the vector $\mathbf{a}(\theta): \mathscr{D} \times \Theta \rightarrow$ $\mathbb{R} n$, where $n$ is the number of FE nodes. The covariance matrix is hence defined as

$$
C_{\mathbf{a}}=\mathbb{E}\left(\mathbf{a}(\theta)-\overline{\mathbf{a}}_{0}\right)\left(\mathbf{a}(\theta)-\overline{\mathbf{a}}_{0}\right)^{T} \quad \text { where } C_{\mathbf{a}} \in \mathbb{R}^{n \times n}
$$

Here, $C_{\mathbf{a}}$ is a Hermitian matrix with identical diagonal terms.

For stationary random fields and a uniformly spaced FE mesh over a one-dimensional spatial domain, it is easy to see that the discrete covariance matrix $C_{\mathbf{a}}$ is a Toeplitz matrix where the entries are constant along the diagonals parallel to the main diagonal. This property can be used efficiently to improve the memory requirement to store a dense covariance matrix, where the entire covariance matrix can be stored with as few as $2 n+1$ elements (compared with the $n(n+1) / 2$ elements required for just symmetric matrices) and with a set of 'forward shift' and 'backward shift' matrices, which are matrices with all elements of the super-diagonal and sub-diagonal, respectively, set to 1 and 0 everywhere else. The case of evaluating the spectral components of a two-dimensional discrete covariance matrix has been investigated in [28] where lexicographic ordering of points has been used in the two-dimensional case and an FFT method is utilized to obtain the eigen-components.

However, the spatial domains $\mathscr{D} \in \mathbb{R} n$, where $n \geqslant 1$, with a non-uniform mesh would not lead to a Toeplitz matrix of spatially discretized covariance matrices. Hence, the eigenvalue problem in Equation (8) becomes more expensive. We apply Lanczos iterative techniques to solve for the largest eigenvalues and write the expression for the rank- $m$ approximation of the covariance operator as

$$
\mathscr{C}_{a}^{(m)}=\Phi \Lambda \Phi^{T}, \quad \text { where } \Phi \in \mathbb{R}^{n \times m} \quad \text { and } \quad \Lambda=\operatorname{diag}\left[\lambda_{1}, \ldots, \lambda_{m}\right]
$$

where $\Phi$ is the matrix of eigenvectors and $\Lambda$ is the diagonal matrix of eigenvalues. The preceding equation holds true for any positive continuous semidefinite kernel on a finite interval owing to Mercer's theorem. Only the first few largest eigen-components are utilized in (12), where $\lambda_{1} \geqslant \ldots \geqslant \lambda_{m}$. This suggests that the covariance kernel is approximated with the desired accuracy using the eigenfunctions obtained from Equation (12). When the correlation length of the random field on the spatial domain is small, higher eigenmodes have to be incorporated into the randomfield approximation. These modes have complex shapes, which implies the necessity of a higher mesh resolution to capture their behavior. Also, Kolmogorov's theorem [29] implies that the realizations of the random fields $a(\mathbf{r}, \theta)$ are Hölder continuous with respect to $\mathbf{r}$ (with Hölder exponent $\alpha<1 / 2$ ) with probability 1 . Hence, the random vectors can be quite irregular. The choice of the discrete points in the spatial domain is crucial in this discussion, as the computational cost of resolving the eigenvalue problem of Equation (8) directly depends on the size of the covariance matrix, while the accuracy requirements may necessitate a high mesh resolution. When the FE nodes are chosen as the discrete points in space, the random field is obtained as $\mathbf{a}(\theta) \in \mathbb{R}^{n_{h}}$, where $n_{h}$ is the dimension of the linear system based on the FE mesh parameter size $h$. The random parameter vector at discrete points in the spatial domain is thus represented as

$$
\mathbf{a}(\theta)=\mathbf{a}_{0}+\sum_{i=0}^{M} \phi_{i} \sqrt{\lambda} \xi_{i}(\theta)
$$

where $\mathbf{a}_{0}$ is the vector of the deterministic parametric field over the spatial domain, while $\operatorname{diag}(\xi(\theta))$ is a diagonal matrix of independent random variables $\xi=\left\{\xi_{1}, \ldots, \xi_{M}\right\}$. It might be mentioned that the covariance matrix may be chosen to be on the quadrature integration points in the spatial domain, which are employed in the weak formulation. This will be discussed in more detail in the next section.

\subsection{Spectral decomposition of the covariance kernel with Galerkin method}

Here, we present the solution methodology of the Fredholm integral equation of the second type in Equation (5) with Galerkin's orthogonal projection technique. This method has been studied in 
[30], and we present it here for the sake of completeness and to compare the computational and implementational details with the proposed discrete method. The methodology in Section 3.1 results in a fully populated symmetric coefficient matrix. However, if higher-order isoparametric elements are used on a coarse FE mesh, the associated discretization error might become undesirable. The Galerkin method can be utilized under these circumstances.

If we define the collection of the set of elements as $\mathscr{E}=\left\{\Delta(\mathscr{D})_{h}: h\right.$ is the mesh parameter size $\}$, then the integral equation in Equation (5) can be rewritten as

$$
\sum_{k \in \mathscr{E} m} \int_{\Omega_{k}^{e}} C_{a}\left(\mathbf{r}_{1_{k}}, \mathbf{r}_{2}\right) \phi_{j}\left(\mathbf{r}_{1_{k}}\right) d \mathbf{r}_{1_{k}}=v_{j} \phi_{j}\left(\mathbf{r}_{2}\right) ; \quad \mathbf{r}_{1_{k}} \in \Omega_{k}^{e}, \quad \mathbf{r}_{2} \in \mathscr{D} \quad \forall j=1,2, \ldots
$$

over each elemental domain $\Omega_{k}^{e}$, and $\mathscr{E}_{m}$ is the cardinality of the set $\mathscr{E}$. We approximate the eigenfunctions $\phi(\mathbf{r})$ in each element domain as $\phi(\mathbf{r})=\sum_{i} \mathscr{N}_{i}(\mathbf{r}) \tilde{\phi}_{i}=[\mathscr{N}(\mathbf{r})]^{T}\{\tilde{\phi}\}$, where $[\mathscr{N}(\mathbf{r})]$ is the vector of the FE shape functions of a chosen degree based on the order of the elements and $\{\tilde{\phi}\}$ is a vector of the functional values of $\phi$ at the discrete FE node points.

A Galerkin projection of the residual of (14) on the $L^{2}(\mathscr{D})$ basis functions [ $\mathscr{N}$ ], that is, $\varepsilon \perp$ $\mathscr{N}_{i}, \forall i$, gives

$$
\begin{aligned}
& \sum_{k \in \mathscr{E}_{m}} \int_{\Omega_{p}^{e}} \int_{\Omega_{k}^{e}}\left[\mathscr{N}\left(\mathbf{r}_{2_{p}}\right)\right] C_{a}\left(\mathbf{r}_{1_{k}}, \mathbf{r}_{2_{p}}\right)\left[\mathscr{N}\left(\mathbf{r}_{1_{k}}\right)\right]^{T}\{\tilde{\phi}\}_{k} d \mathbf{r}_{1_{k}} d \mathbf{r}_{2_{p}} \\
& \quad=v_{j} \int_{\Omega_{p}^{e}}\left[\mathscr{N}\left(\mathbf{r}_{2_{p}}\right)\right]\left[\mathscr{N}\left(\mathbf{r}_{2_{p}}\right)\right]^{T}\{\tilde{\phi}\}_{p} d \mathbf{r}_{2_{p}} \quad \forall p \in \mathscr{E}_{m}
\end{aligned}
$$

The preceding equations result in fully populated coefficient matrices, which is similar to the discrete KL expansion approach in Section 3.1.

Alternatively, the covariance function inside the elemental domains can be approximated with the spatial interpolation functions. If $C_{a p k}^{e}$ is the covariance function constructed between the elements $p$ and $k$, we write

$$
C_{e}^{a_{p k}}\left(\mathbf{r}_{1_{k}}, \mathbf{r}_{2_{p}}\right)=\left[\mathscr{N}\left(\mathbf{r}_{1_{k}}\right) \otimes \mathscr{N}\left(\mathbf{r}_{2_{p}}\right)\right]: \mathscr{C}^{e}\left(r_{i}, r_{j}\right)=\left[\mathscr{N}\left(\mathbf{r}_{2_{p}}\right)\right]^{T} \mathscr{C}^{e}\left(r_{i_{k}}, r_{j_{p}}\right)\left[\mathscr{N}\left(\mathbf{r}_{1_{k}}\right)\right]
$$

where $\mathscr{C}\left(r_{i}, r_{j}\right) \in \mathbb{R}^{n_{e} \times n_{e}}$ is the discrete covariance matrix. Application of the Galerkin orthogonal projection gives the element-level matrices as

$$
\mathbf{a}_{p k}^{e}=\mathbf{M}_{p}^{e} \mathscr{C}^{e}\left(r_{i_{k}}, r_{j_{p}}\right) \mathbf{M}_{k}^{e}
$$

where $\mathbf{M}^{e}=\left(\int_{\Omega^{e}}[\mathscr{N}(\mathbf{r})][\mathscr{N}(\mathbf{r})]^{T} d \mathbf{r}\right)$ are the equivalent of element-level mass matrices. Taking Equations (15)-(17) together, we have the discretized linear system of the form

$$
\mathbf{A} \phi=v \mathbf{M} \phi
$$

where $\mathbf{A}$ is a fully populated symmetric matrix and $\mathbf{M}$ is obtained from the right-hand side of Equation (15). The preceding equation gives a generalized eigenvalue problem. The eigenvalue problem in Equation (18) involves a dense matrix $\mathbf{A}$, which is expensive for large systems. Hence, while Galerkin's method can enhance the approximation accuracy of the solution, it involves a higher computational cost for obtaining the linear system for the generalized eigenvalue problem with no benefits in terms of the memory requirement or using an efficient algorithm to resolve the dominant eigenmodes of the covariance functions. 
3.2.1. Discrete representation of the lognormal field model. The Gaussian random-field models, while offering an easier scheme for computational implementation, is often unsuitable for modeling the uncertainty for those physical systems where the parametric variation has to be considered strictly positive to make any practical sense. Under such circumstances, the lognormal field is generally the favored distribution and has been adopted in the present work. The discrete KL expansion framework laid out in the previous sections can easily be extended to incorporate this kind of random-field distributions. A lognormal field of the discretized random field $\mathbf{a}_{l}(\theta) \in \mathbb{R} n \times \Theta^{(M)}$ is expressed as the exponential of the corresponding Gaussian-field model $\mathbf{a}(\theta)$. Following from the discretized KL expansion in Equation (13),

$$
\mathbf{a}_{l}(\theta)=\exp \left(\overline{\mathbf{a}}_{0}+\sum_{i=1}^{M} \xi_{i}(\theta) \tilde{\mathbf{a}}_{i}\right)
$$

where $\xi=\left\{\xi_{1}, \ldots, \xi_{M}\right\}$ is the vector of independent and identically distributed (i.i.d.) Gaussian random variables. To make the preceding expression computationally favorable, the lognormal field is often expressed as a finite-order multivariate expansion of the basic i.i.d. random variables from the Weiner-Askey scheme. When the basic i.i.d. random fields are Gaussian in nature, the polynomial expansion of the lognormal field is

$$
\mathbf{a}_{l}=\sum_{i=0}^{m} \mathbf{a}_{i}^{l} \mathscr{H}_{i}(\xi(\theta)), \quad \text { where } \quad \mathbf{a}_{i}^{l}=\frac{\left\langle\mathbf{a}_{l}(\theta), \mathscr{H}_{i}(\xi(\theta)\rangle_{L^{2}\left(\Theta^{(M)}, d P_{\xi}\right)}\right.}{\left\langle\mathscr{H}_{i}(\xi(\theta)\rangle_{L^{2}\left(\Theta^{(M)}, d P_{\xi}\right)}^{2}\right.}
$$

where $\mathscr{H}_{i}(\xi(\theta))$ are the multivariate Hermite polynomials spanning the stochastic space $\theta \in \Theta^{(M)}$ and $\langle\cdot, \cdot\rangle_{L^{2}\left(\Theta^{(M)}, d P_{\xi}\right)}$ denotes the inner product in the same space. The value of $m$ is guided by the dimension of the input polynomial space $M$ and the chosen order of expansion $p$ as $m=\left(\begin{array}{c}M+p \\ p\end{array}\right)$. The $L^{2}$ norm of the Hermite polynomials are easily available, generally precomputed and fed into the solver. The inner products essential for the evaluation of the numerator in Equation (20) can be computed using the analytical expression applicable for Gaussian fields as

$$
\mathbf{a}_{i}^{l}=\exp \left(\overline{\mathbf{a}}_{0}\right) \prod_{i=1}^{M} \exp \left(\tilde{\mathbf{a}}_{i}^{2}\right) \frac{\mathscr{H}_{i}^{h d}\left(\tilde{\mathbf{a}}_{1}, \ldots, \tilde{\mathbf{a}}_{M}\right)}{\left\langle\mathscr{H}_{i}(\xi(\theta)\rangle_{L^{2}\left(\Theta^{(M)}, d P_{\xi}\right)}^{2}\right.}
$$

where $\mathscr{H}_{i}^{\text {hd }}\left(\mathbf{a}_{1}, \ldots, \mathbf{a}_{M}\right)$ denotes the highest-order term associated with the $i$ th Hermite polynomial and the arguments are replaced by the coefficients of the random variables (which are the eigenmodes obtained from the discretized KL expansion) instead of the random vector $\xi=\left\{\xi_{1}, \ldots, \xi_{M}\right\}$ itself. The proof of this has been provided in Appendix A. This provides an efficient analytical way to compute the undetermined coefficients associated with the lognormal random field given in Equation (20) instead of a multidimensional integration in the stochastic subspace for each coefficient.

\section{STOCHASTIC WEAK FORMULATION WITH DISCRETE RANDOM PARAMETER}

Here, we consider the FE spaces associated with the spatial and stochastic dimensions of the randomly parameterized system laid out in the previous sections. The spatial set $\mathscr{D} \in \mathbb{R}^{d}$ and the set of random outcomes modeled in the finite-dimensional stochastic space $\Theta^{(M)} \subset \Theta$ will be used to define the tensor product space $\mathscr{D} \times \Theta^{(M)}$, where the solution to the stochastic weak formulation is sought. 


\subsection{Finite element spaces}

The spaces involved in the construction of the stochastic FE method (SFEM) for the stochastic weak formulation is presented in $[31,32]$, which gives two paradigms in which the solution of the preceding problem is sought: (i) the $k \times h$-SFEM version and the (ii) the $p \times h$-SFEM version. We consider these in detail here: let the spatial domain $\mathscr{D}$ be meshed as $\Delta(\mathscr{D})$ such that the mesh parameter size is given by $h(\Delta(\mathscr{D}))$. The finite-dimensional stochastic domain may be partitioned with a finite number of disjoint boxes having finite intervals along each dimension, and we denote this rectangular mesh as $\Delta\left(\Theta^{(M)}\right)=\Delta\left(\Theta_{1}\right) \times \ldots \times \Delta\left(\Theta_{M}\right)$. Thus, the mesh on the tensor product space is denoted as $\Delta\left(\mathscr{D}, \Theta^{(M)}\right)$, and the elements are denoted as $\tau\left(\Delta\left(\mathscr{D}, \Theta^{(M)}\right)\right)=\tau(\Delta(\mathscr{D})) \times$ $\tau\left(\Delta\left(\Theta^{(M)}\right)\right)$. The approximating functions on this tensor product space are given by the set

$$
\begin{aligned}
S^{p, q}\left(\mathscr{D}, \Theta^{(M)}\right)= & \left\{v(\mathbf{r}, \xi) \in W|v(\mathbf{r}, \xi)|_{\tau\left(\Delta\left(\mathscr{D}, \Theta^{(M)}\right)\right)} \text { is a polynomial of degree } p \text { in } \mathbf{r},\right. \\
\forall \xi \in \theta \text { and of degree } q \text { in } \xi, \forall \mathbf{r} \in \mathscr{D}\} & \\
\text { where } W|v(\mathbf{r}, \xi)|_{\mathscr{D}, \Theta^{(M)}}= & \left\{v(\mathbf{r}, \theta): \int_{\Theta^{(M)}}\|v(\mathbf{r}, \xi)\|_{L^{2}(\mathscr{D})} d P_{\xi}(\theta) \leqslant \infty,\right. \\
& \left.\theta \in \Theta^{(M)} \text { and } v(\mathbf{r}, \xi)=0 \text { on } \partial \mathscr{D}, \forall \xi \in \theta\right\}
\end{aligned}
$$

is the space of polynomials, which converge in the $L^{2}\left(\Theta^{(M)}, d P_{\xi} ; \mathscr{D}\right)$ sense. The definition of the $L^{2}$ norm depends on the physical problem at hand (e.g., when we have an elliptic stochastic differential operator, $\left.\|v(\mathbf{r}, \xi)\|_{L^{2}(\mathscr{D})}=\int_{\Delta(\mathscr{D})} a(\mathbf{r}, \theta)\left|\nabla_{\mathbf{r}} v(\mathbf{r}, \xi)\right|^{2} d \mathbf{r}\right)$. The assumptions on the chosen random parameter $a(\mathbf{r}, \theta)$ and its joint probability distribution render $W$ a Hilbert space. For the standard deterministic FEs, the weak form of the governing partial differential equations is stated as $b(u(\mathbf{r}), v(\mathbf{r}))=l(v(\mathbf{r})) \forall v \in L^{2}(\mathscr{D})$, where $u$ is the solution that is sought, $v$ consists of the test functions in the admissible $L^{2}$ space, and $b$ and $l$ are the continuous bilinear and linear forms, respectively, on the spatial domain $\mathbf{r} \in \mathscr{D}$. Thus, the bilinear and linear forms associated with the weak stochastic FE formulation is written as

$$
\mathscr{B}(u, v)=\int_{\Theta(M)} b(u(\mathbf{r}, \theta), v(\mathbf{r}, \theta) ; \theta) d P_{\xi}(\theta) ; \quad \mathscr{L}(v)=\int_{\Theta(M)} l(v(\mathbf{r}, \theta) ; \theta) d P_{\xi}(\theta)
$$

so that $\mathscr{B}(u, v)=\mathscr{L}(v)$

The basic FE theory ensures the existence and uniqueness of the solution $u_{S^{p, q}} \in S^{p, q}\left(\mathscr{D}, \Theta^{(M)}\right)$ and the convergence to the actual solution as

$$
\text { and } \begin{aligned}
&\left\|u-u_{S^{p, q}}\right\|_{W\left(\mathscr{D}, \Theta^{(M)}\right)} \rightarrow 0 \text { as } h(\Delta(\mathscr{D})), k\left(\Delta\left(\Theta^{(M)}\right)\right) \rightarrow 0 \\
& \text { and } \quad\left\|u-u_{S^{p, q}}\right\|_{W\left(\mathscr{D}, \Theta^{(M)}\right)} \rightarrow 0 \text { as } p, q \rightarrow \infty
\end{aligned}
$$

The preceding equations stand for the convergence of the $h$-version and the $p$-version of the SFEM, respectively. The $p \times h$-SFEM seeks the solution in tensor product space $\Theta^{(M), q} \otimes \Delta\left(\mathscr{D}_{h}\right)$ and produces exponential convergence of the solution with $p$ [33]. We consider here this latter version of the weak formulation where the input stochastic space has been discretized with a finite set of i.i.d. random variables and the solution at the nodal points in the discretized spatial domain is expressed with a finite $p$ th-order polynomial function of the random variables.

\subsection{Description of the stochastic steady-state diffusion problem}

Let us consider here a steady-state diffusion problem on an arbitrary domain in the threedimensional space, and the diffusion coefficient $K$ is assumed to be uncertain. If we consider the heat 
transport problem, then $K$ becomes the thermal diffusivity. The governing PDE of the steady-state diffusion problem along with the Dirichlet boundary conditions may be written as

$$
\nabla \cdot(K(\mathbf{r} ; \theta) \nabla u)=Q(\mathbf{r}) \quad \text { and } \quad u=0 \text { on } \partial \mathscr{D}
$$

where $Q$ is the source/sink term in the domain. When formulating the weak form of the preceding system at a particular point in the stochastic sample space $\theta$, we have

$$
\begin{aligned}
& b(u, v ; \theta)=l(v ; \theta) \quad \text { where } \\
& b(u, v ; \theta)=\int_{\mathscr{D}} K(\mathbf{r}, \theta)(\nabla v) \cdot(\nabla u) d \mathscr{D} ; \quad \& \quad l(v ; \theta)=\int_{\mathscr{D}} v Q d \mathscr{D}
\end{aligned}
$$

Here, $K$ is modeled with a finite set of random variables following the discrete KL expansion (detailed in Section 3.1) of the covariance kernel associated with the random field on the domain such that

$$
K(\theta)=\bar{K}_{0}+\sum_{j=1}^{m} \mathscr{H}_{j}(\xi(\theta)) K_{j} ; \quad K(\theta) \in \mathbb{R} \times \Theta^{(M)} \rightarrow \mathbb{R} n
$$

is expressed by its mean $\bar{K}_{0}$ and perturbation components $K_{j}$ in the series expansion form. The spatial discretization of the random parameter may be performed using the same mesh that is used for the resolution of the response of the FE system, that is, $\Delta(\mathscr{D})$, and the vector $K(\theta)$ is the parameter values at the FE nodes. Here, we introduce the following FE discretization spaces: (i) $\bar{X}^{q}\left(\Theta^{(M)}\right) \subset L^{2}\left(\Theta^{(M)}\right)$ such that $\bar{X}^{q}\left(\theta_{i}\right) \subset L^{2}\left(\theta_{i}\right)$ consists of up to $q$ th-order polynomials in $L^{2}\left(\theta_{i}\right)$ with $\Theta^{(M)}=\prod_{i=1}^{M} \theta_{i} \subset \mathbb{R}^{M}$ and the probability density support $P(\xi)=\prod_{i=1}^{M} P\left(\xi_{i}\right) \xi_{i} \in$ $\theta_{i} \forall i$ and (ii) $H_{0}^{1}\left(\Delta\left(\mathscr{D}_{h}\right)\right)$ consists of all functions, which vanishes on the boundary with the norm $\|v\|=\left\{\int_{\Delta(\mathscr{D})}|\nabla v|^{2} d \mathbf{r}\right\}^{1 / 2}$. Thus, the approximation space of the test functions $v_{e}$ in $\Theta^{(M), q} \times \Delta\left(\mathscr{D}_{h}\right)$, which is meshless in $\Theta^{(M)}$, for the weak form is given as

$$
\begin{aligned}
\bar{X}^{q}\left(\Theta^{(M)}\right) \otimes H_{0}^{1}\left(\Delta\left(\mathscr{D}_{h}\right)\right)= & \left\{v_{e}=v_{e}(\mathbf{r}, \xi) \in L^{2}\left(\Theta^{(M)} \times \Delta\left(\mathscr{D}_{h}\right)\right):\right. \\
& \left.v_{e} \in \operatorname{span}\left(\mathscr{N}\left(\mathbf{r}_{h}\right) \mathscr{H}(\xi(\theta)): \mathscr{N} \in H_{0}^{1}, \mathscr{H} \in \bar{X}^{q}\right)\right\}
\end{aligned}
$$

where $\Delta\left(\mathscr{D}_{h}\right)$ denotes the mesh with the mesh parameter size $h$. This corresponds to the $p \times h$-SFEM version. We expand the solution in each discretized element $\Theta^{(M), q} \otimes \Delta\left(\mathscr{D}_{h}\right)$ as

$$
u_{e}\left(\mathbf{r}_{h}, \theta\right)=\sum_{i=1}^{n_{e}} u_{i}^{q, M}(\theta) \mathscr{N}_{i}^{p}\left(\mathbf{r}_{h}\right) ; \quad \mathbf{r}_{h} \in \tau(\Delta(\mathscr{D})), \quad \theta \in \Theta^{(M), q}
$$

where $p$ and $q$ denote the order of the polynomials of the spatial and stochastic basis functions, respectively, $M$ is the dimension of the input stochastic space, $h$ is the mesh size parameter, and $\mathscr{N}_{i}^{p}\left(\mathbf{r}_{h}\right)$ are the multidimensional Lagrange basis functions of order $p$ on $\mathbf{r}_{h}$. For the $p \times h$-SFEM, we have the vector of random coefficients $\mathbf{u}_{e}^{q, M}(\theta)=\left\{u_{1}^{q, M}, \ldots, u_{n_{e}}^{q, M}\right\}$ expressed with polynomial functions $\mathscr{H}(\xi(\theta))$ of the input i.i.d. random variables. Here, $\mathbf{u}_{e}^{q, M} \in \mathbb{R}^{n_{e}}$, where $n_{e}$ is the number of nodes associated with each element of the FE mesh.

The vector of the random parameter values at the nodal points is interpolated inside the spatial domain of the elements using the deterministic FE shape functions, which are the basis functions for the expansion of the stochastic system response in the spatial domain, that is, $\mathscr{N}_{i}^{p}\left(\mathbf{r}_{h}\right)$. The random parameter has already been expressed in the stochastic domain with the global stochastic polynomial basis as per Equation (13). Then the stochastic bilinear operator associated with the weak form on each spatial element of the FE mesh $\Delta(\mathscr{D})$ and the global stochastic function space $\Theta^{(M)}$ may be written as (following from Equations (24) and (26)) 


$$
\mathscr{B}_{\Theta(M), q \otimes \Delta\left(\mathscr{D}_{h}\right)}\left(u_{e}, v_{e}\right)=\int_{\Theta^{(M)}} \int_{\Delta\left(\mathscr{D}_{h}\right)}\left[\mathscr{N}^{p}\left(\mathbf{r}_{h}\right)\right]^{T}\left[K_{e}(\theta)\right]\left(\nabla v_{e}\right) \cdot\left(\nabla u_{e}\right) d \mathscr{D} d P_{\xi}(\theta)
$$

where $\left[\mathscr{N}^{p}\right]=\left\{\mathscr{N}_{1}^{p}, \ldots, \mathscr{N}_{P}^{p}\right\}$ is the vector of the spatial basis functions (of order $p$ ) and $\left[K_{e}(\theta)\right]$ is the vector of the random parameter at the nodal points belonging to the element $\tau\left(\Delta\left(\mathscr{D}_{h}\right)\right)$. The linear form accordingly becomes

$$
\mathscr{L}_{\Theta^{(M), q} \otimes \Delta\left(\mathscr{D}_{h}\right)}\left(v_{e}\right)=\int_{\Theta^{(M)}} \int_{\Delta\left(\mathscr{D}_{h}\right)} v_{e} Q\left(\mathbf{r}_{h}\right) d \mathscr{D} d P_{\xi}(\theta)
$$

The element-level equations take the following form:

$$
\mathscr{B}_{\Theta(M), q \otimes \Delta\left(\mathscr{D}_{h}\right)}\left(u_{e}, v_{e}\right)=\mathscr{L}_{\Theta(M), q \otimes \Delta\left(\mathscr{D}_{h}\right)}\left(v_{e}\right)
$$

The perturbation matrices of the FE system are then given as

$$
\mathbf{A}_{i}^{(e)}=\sum_{i=1}^{d} \int_{\Delta\left(\mathscr{D}_{h}\right)}\left[\mathscr{N}^{p}\left(\mathbf{r}_{h}\right)\right]^{T}\left[K_{e, i}\right]\left[\frac{\partial \mathscr{N}^{p}\left(\mathbf{r}_{h}\right)}{\partial \eta_{i}}\right]\left[\frac{\partial \mathscr{N}^{p}\left(\mathbf{r}_{h}\right)}{\partial \eta_{i}}\right]^{T} d \mathscr{D}
$$

where $d$ denotes the dimension of the Euclidean space in which the physical domain of the problem exists, that is, $\mathscr{D} \in \mathbb{R}^{d}, \eta_{i}$ is the $i$ th coordinate axis in the same Euclidean space, and $K_{e, i}$ is the $i$ th term in the series expansion of the random parameter following Equation (13).

Equation (33) can be extended to include the linear or isoparametric mapping for a spatial domain meshed with non-uniform elements. For example, if we consider the mapping of the Cartesian coordinate axes $\eta_{i}$ to the parameter space, we have

$$
\eta_{i}=Q_{i}\left(\tilde{\eta}_{1}, \ldots, \tilde{\eta}_{d}\right) \quad \forall i=1, \ldots, d ; \quad \text { and } \quad \Delta\left(\mathscr{D}_{h}\right)=\overline{\mathbf{Q}}(\Delta(\mathbf{D}))
$$

where $\tilde{\eta}=\left\{\tilde{\eta}_{1}, \ldots, \tilde{\eta}_{d}\right\}$ are the set of parent axes with $\Delta(\mathbf{D})$ being the parent hypercube onto which the elements $\Delta\left(\mathscr{D}_{h}\right)$ are mapped using the transformation $\overline{\mathbf{Q}}$. The system matrices can then be written in terms of the isoparametric integration as

$$
\mathbf{A}_{i}^{(e)}=\sum_{i=1}^{d} \int_{\Delta(\mathbf{D})}\left[\mathscr{N}^{p}(\tilde{\eta})\right]^{T}\left[K_{e, i}\right]\left[\frac{\partial \mathscr{N}^{p}(\tilde{\eta})}{\partial \eta_{i}}\right]\left[\frac{\partial \mathscr{N}^{p}(\tilde{\eta})}{\partial \eta_{i}}\right]^{T}|\mathbf{J}(\tilde{\eta})| d \mathbf{D}
$$

where $\quad J_{i j}(\tilde{\eta}) \stackrel{\text { def }}{=} \frac{\partial \eta_{i}}{\partial \tilde{\eta}_{j}} \quad \forall i, j \in[1, \ldots, d]$

that is, $|\mathbf{J}(\tilde{\eta})|$ is the determinant of the Jacobian matrix, and $d \mathbf{D}=d \tilde{\eta}_{1} \ldots d \tilde{\eta}_{d}$. The preceding integral is usually evaluated with a finite-order Gauss-Legendre quadrature. The order is guided by the degree of polynomial involved in the integration. When the integration is performed with $n$ points, Gauss-Legendre quadrature produces exact integrals for all polynomials of order up to $2 n-1$. The interpolating shape functions used to express the KL eigenmodes within the element domain increase the polynomial order, and hence, more Gauss points are necessary to compute the perturbation parts of the diffusion matrix, compared with its deterministic counterpart $\mathbf{A}_{0}^{(e)}$. Let us denote the grid of $n^{d}$ Gauss points in the parent hypercube as $\mathscr{G}_{\hat{\eta}}=\left\{\hat{\eta}_{m}: \hat{\eta}_{m}=x_{j_{1} \ldots j_{d}} \forall j_{1}, \ldots, j_{d} \in\{1, \ldots, n\}\right.$ and $\left.m=1, \ldots, n^{d}\right\}$ with the associated weights $\mathscr{G}_{\tilde{W}}=\left\{\tilde{W}_{m}: \tilde{W}_{m}=\tilde{w}_{j_{1}} \ldots \tilde{w}_{j_{d}} \forall j_{1}, \ldots, j_{d} \in\{1, \ldots, n\}\right.$ and $\left.m=1, \ldots, n^{d}\right\}$. The expression for the integral in terms of the Gauss quadrature points is

$$
\mathbf{A}_{i}^{(e)}=\sum_{i=1}^{d} \sum_{m \in \mathscr{I}\left(\mathscr{G}_{p}\right)} \tilde{W}_{m}\left[\mathscr{N}^{p}(\tilde{\eta})\right]_{\tilde{\eta}=\hat{\eta}_{m}}^{T}\left[K_{e, i}\right]\left[\frac{\partial \mathscr{N}^{p}(\tilde{\eta})}{\partial \eta_{i}}\right]_{\tilde{\eta}=\hat{\eta}_{m}}\left[\frac{\partial \mathscr{N}^{p}(\tilde{\eta})}{\partial \eta_{i}}\right]_{\tilde{\eta}=\hat{\eta}_{m}}^{T}|\mathbf{J}(\tilde{\eta})|_{\tilde{\eta}=\hat{\eta}_{m}}
$$


where $\mathscr{I}\left(\mathscr{G}_{\hat{\eta}}\right)$ is the cardinality of the set $\mathscr{G}_{\hat{\eta}}$. The derivative of the shape functions $\mathscr{N}^{p}$ with respect to the global coordinates $\tilde{\eta}$ has to go through a coordinate transformation, which is written in terms of the inverse of the Jacobian matrix denoted by $[\mathbf{J}(\tilde{\eta})]^{-1}$, which is well established in the isoparametric FE literature [24]. From Equation (36), it is seen that we are trying to evaluate the random parameter $K_{e}$ at the quadrature integration points within the element domain. Hence, a better approximation of the random field would be obtained if the discrete spectral decomposition of the covariance function in Equation (12) is performed such that the eigenvector $\Phi$ is evaluated at the quadrature integration points. However, the additional computation cost has to be justified by the significance of the improvement in accuracy of the obtained solution. The next section deals with the solution methodology applied to the preceding discretized system to propagate the input parametric uncertainty to the system response and the evaluation of the second-order response statistics.

\subsection{Solution methodology}

Here, we present the solution methodology adopted in this study to solve the stochastic diffusion problem of the FE discretized system. We present the setting for the Galerkin method with polynomial chaos expansion, which has been used to represent the steady-state diffusion equation with a finite-order chaos expansion [30, 34]. As has been discussed in the literature (e.g., [35]), the dimensionality of the linear system increases significantly with the order of chaos chosen and the dimension of the input stochastic space. Other efficient uncertainty propagation methods include stochastic collocation [6] and polynomial dimensional decomposition [36, 37] to name a few.

The assembly of the aforementioned element matrices to form the stochastic global system can be written as $\mathbf{A}(\theta)=\sum_{i=0}^{m} \mathscr{H}_{i}(\xi(\theta))\left[\bigcup_{e \in \mathscr{E}} \mathbf{A}_{i}^{(e)}\right]$ from Equation (36), where $\mathbf{A}_{i}^{(e)}$ is the elementlevel (e) matrix associated with the $i$ th KL mode of the random field, $\mathscr{E}$ is the set of all elements of the FE mesh on the spatial domain, and $\mathscr{H}(\xi(\theta))$ are the stochastic basis functions, which express the random field with i.i.d. random variables $\xi(\theta)$. Hence, the Galerkin formulation at the stochastic level gives

$$
\mathbb{E}\left[\left[\mathbf{v}^{q, M}\right]^{T} \mathbf{A}(\theta) \mathbf{u}^{q, M}\right]=\mathbb{E}\left[\left[\mathbf{v}^{q, M}\right]^{T} \mathbf{Q}\right] \text { where } \mathbf{A}: \Theta^{(M)} \rightarrow \mathbb{R}^{n \times n} ; \quad \forall \mathbf{v}^{q, M} \in \mathbb{R}^{n} \times L^{2}\left(\Theta^{(M)}, d P_{\xi}\right)
$$

where $\mathbb{E}[\bullet]$ is the expectation operator on the probability space, $\mathbf{u}^{q, M}$ is an assembly of the element block vectors $\mathbf{u}_{e}^{q, M}$ given in Equation (29), and $\mathbf{v}^{q, M}$ is the stochastic trial vector basis. Equation (37) leads to the following set of equations:

$$
\sum_{\alpha \in \mathscr{I}_{q, M}} \mathbb{E}\left[\mathbf{A}(\theta) \mathscr{H}_{\alpha}(\theta) \mathscr{H}_{\beta}(\theta)\right] \mathbf{u}_{\alpha}^{q, M}=\mathbb{E}\left[\mathscr{H}_{\beta} \mathbf{Q}\right]
$$

If we denote $\underline{\mathbf{U}}=\left\{\mathbf{u}_{\alpha}^{q, M}: \alpha \in \mathscr{I}_{q, M}\right\}$, we have

$$
\underline{\mathbf{A}} \underline{\mathbf{U}}=\underline{\mathbf{Q}}
$$

where $\underline{\mathbf{A}}$ is the block-sparse system coefficient matrix and $\mathbf{Q}$ is the right-hand block vector. The block-sparse nature of the coefficient matrix is due to the fact that the Galerkin orthogonalization of the residual to the stochastic basis functions involves an integration in the stochastic space with the terms $H_{\alpha \beta \gamma}=\mathbb{E}\left[\mathscr{H}_{\alpha}(\theta) \mathscr{H}_{\beta}(\theta) \mathscr{H}_{\gamma}(\theta)\right]$. The $H_{\alpha \beta \gamma}$ is zero for most combinations of $\{\alpha, \beta, \gamma\}$. Another efficient alternative is to use double orthogonal polynomials for the random field, in which case the coefficient matrix would be block-diagonal and the stochastic problem would be decoupled [31]. Detailed discussion on the efficient solution of the linear system in Equation (39) is beyond the scope of the current work, and hence, the reader is referred to a review of the important literature in this domain $[38,39]$. 


\section{RANDOM BOUNDARY ROUGHNESS}

Surface roughness has been accounted for by using various methods ranging from simple parameterization of surface inhomogeneities $[40,41]$ to use of fractals to represent the perturbations in the domain [42]. An alternative approach is to assume that the detailed boundary topology is uncertain and to use random fields to model it. This description has been used in a number of studies that are briefly discussed here. A solution of PDEs on random domains was investigated in [12] using a stochastic mapping of the body-fitted curvilinear coordinates. The extended FE method has been extended to PDEs on random domain [43] and for tackling random heterogeneous material interfaces [44]. This concept utilizes a level-set technique to implicitly represent the random geometry and then uses the classical spectral SFEM to solve the problem on a fixed FE mesh. The method relies on representing the geometry and its randomness implicitly with random level-set functions. However, incorporating the randomness into the complex geometries with stochastic parameterization of random level-set functions is not always a trivial exercise. Moreover, the random boundary may be realized with a random-field model rather than just using random variables to model the stochastic level-set functions. The method proposed in this work can potentially overcome these difficulties. Another study [45] uses the Laplace equation-based mesh deformation approach to model the stochastic domain with random variables and uses the stochastic collocation scheme to obtain the element matrices. This non-intrusive approach does away with the complexity associated with the formation of the system matrices but is computationally expensive, and the error associated with the formation of the system-level matrices has to be controlled carefully. Literature also exists on natural convection with random boundary topology with a sparse-grid collocation technique [6] and investigation of transport phenomenon in rough walled tubes [8]. However, integrating the treatment of these uncertainties in a generic fashion within the stochastic spectral FE literature still remains a challenge. This is because the stochastic mappings involved in these cases are particularly cumbersome to incorporate within the SFEM framework.

\subsection{Problem definition in the random domain}

A diffusion problem is considered here on a domain with random boundary perturbations such that $\mathscr{D}_{\theta} \subset \mathbb{R}^{d}$, where $\theta$ signifies the randomness component. Hence,

$$
\begin{aligned}
& \nabla(K \nabla u)=Q \quad \text { on } \mathscr{D}_{\theta} \\
& u=G_{c} \text { on } \Gamma_{\theta}^{0} \\
& \mathbf{n} \cdot \nabla u=q_{c} \quad \text { on } \Gamma_{\theta}^{1} \quad \text { and } \quad \mathbf{n} \cdot \nabla u=0 \quad \text { on } \Gamma_{\theta}^{2}
\end{aligned}
$$

where $\Gamma_{\theta}^{1}$ and $\Gamma_{\theta}^{2}$ belong to a part of the boundary $\partial \mathscr{D}_{\theta}$ that is random and $\Gamma_{\theta}^{1} \cap \Gamma_{\theta}^{2}=\varnothing$. Here, $\mathbf{n}_{1}$ and $\mathbf{n}_{2}$ are the outward normals to the random boundary $\Gamma_{\theta}^{1}$ and $\Gamma_{\theta}^{2}$, respectively. The preceding equations indicate that the field values are prescribed on the boundary for every sample realization of the random boundary, which implies $G_{c}$ and $q_{c}$ are the same for every random sample.

The weak formulation of the problem in Equation (40) expressed on the FE mesh $\Delta\left(\mathscr{D}_{\theta}\right)$ is such that the solution is sought in $\overline{\mathbf{u}}_{\theta}=\left\{v_{e}\left(\chi^{\theta}\right)=\mathscr{N}\left(\chi^{\theta}\right) \mathbf{v}_{\theta} ; \mathbf{v}_{\theta} \in \mathbf{R}^{n}\right\}$, where $\mathscr{N}\left(\chi^{\theta}\right)$ consists of spatial basis functions. For the sake of simplicity, if we consider $\Gamma_{1}$ to be a zero Neumann boundary for now, the following bilinear and linear forms are obtained:

$$
\begin{gathered}
\mathscr{B}_{\Delta\left(\mathscr{D}_{\theta}\right)}\left(u_{e}, v_{e}\right)=\int_{\Delta\left(\mathscr{D}_{\theta}\right)} K_{e}\left(\nabla_{\chi^{\theta}} \mathscr{N} \mathbf{v}\right) \cdot\left(\nabla_{\chi^{\theta}} \mathscr{N} \mathbf{u}\right) d \mathscr{D}_{\theta} \text { and } \mathscr{L}_{\Delta\left(\mathscr{D}_{\theta}\right)}\left(v_{e}\right)=\int_{\Delta\left(\mathscr{D}_{\theta}\right)}(\mathscr{N} \mathbf{v}) Q d \mathscr{D}_{\theta} \\
\text { such that } \mathscr{B}_{\Delta\left(\mathscr{D}_{\theta}\right)}\left(u_{e}, v_{e}\right)=\mathscr{L}_{\Delta\left(\mathscr{D}_{\theta}\right)}\left(v_{e}\right)
\end{gathered}
$$

In the classical isoparametric $\mathrm{FE}$ formulation of the preceding integrals, the element domain is transformed into a master domain (e.g., the domain $A^{\prime \prime} B^{\prime \prime} C^{\prime \prime} D^{\prime \prime}$ is transformed into $A B C D$ as in Figure 2). Thus, the integration is transformed into the master domain, which can map any order of arbitrary-shaped elements to the same order of a regular-shaped parent element. This facilitates 

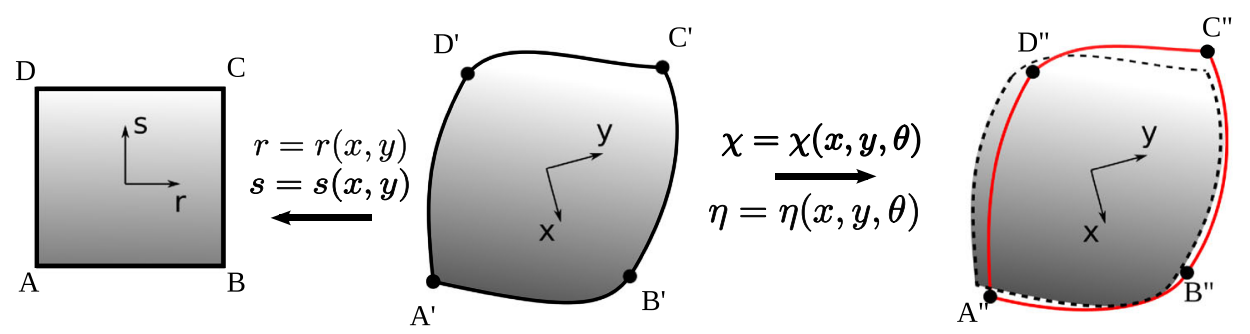

Figure 2. A quadrangular element from the finite element unstructured mesh in its perturbed configuration (marked in red). The leftmost figure denotes the master element, and the middle figure denotes the deterministic element.

easy implementation of a numerical quadrature scheme. It involves evaluating a Jacobian matrix $\mathbf{J}_{\theta}$, which transforms the differential volume $d \mathscr{D}_{\theta}$ in each realization of the random domain into its master domain as $d \mathscr{D}_{\theta}=\mathbf{J}_{\theta} d \mathscr{D}_{M}$. Also, the differential operator undergoes a transformation as $\nabla_{\chi^{\theta}}=\mathbf{J}_{\theta}^{-1} \nabla_{\eta}$. In the isoparametric FE formulation [46], the degree $p$ of the shape functions used to approximate the response field is the same as that used for the map of a given domain to its master domain. Hence, Equation (41) can be written as

$$
\begin{aligned}
\mathscr{B}_{\Delta\left(\mathscr{D}_{\theta}\right)}\left(u_{e}, v_{e}\right) & =\int_{\Delta\left(\mathscr{D}_{M}\right)} K_{e}\left(\mathbf{J}_{\theta}^{-1} \nabla_{\tilde{\eta}} \mathscr{N}^{p}(\tilde{\eta}) \mathbf{v}\right) \cdot\left(\mathbf{J}_{\theta}^{-1} \nabla_{\tilde{\eta}} \mathscr{N}^{p}(\tilde{\eta}) \mathbf{u}\right) \mathbf{J}_{\theta} d \mathscr{D}_{M} \\
\mathscr{L}_{\Delta\left(\mathscr{D}_{\theta}\right)}\left(v_{e}\right) & =\int_{\Delta\left(\mathscr{D}_{M}\right)}\left(\mathscr{N}^{p}(\tilde{\eta}) \mathbf{v}\right) Q \mathbf{J}_{\theta} d \mathscr{D}_{M}
\end{aligned}
$$

such that $\mathscr{B}_{\Delta\left(\mathscr{D}_{\theta}\right)}\left(u_{e}, v_{e}\right)=\mathscr{L}_{\Delta\left(\mathscr{D}_{\theta}\right)}\left(v_{e}\right)$

Here, the Jacobian $\mathbf{J}_{\theta}$ is specific to each realization of the random boundary and has to be calculated for the resolution of the system at each point in the stochastic space. The Jacobian takes the form of $\mathbf{J}_{\theta}=\partial \chi^{\theta} / \partial \tilde{\eta}$, where $\chi^{\theta}=\left\{\chi_{1}^{\theta}, \ldots, \chi_{d}^{\theta}\right\}$ denotes the vector of coordinate directions for each realization of the random domain and $\tilde{\eta}=\left\{\tilde{\eta}_{1}, \ldots, \tilde{\eta}_{d}\right\}$ is the same in the master domain. The preceding equation can be solved in this form using non-intrusive approaches such as various Monte Carlo simulations, response surface methodologies, sparse-grid collocation techniques [6], or hybrid metamodeling techniques [47],

An alternative to this approach is to solve the system using a unified stochastic Galerkin approach where the solution can be expanded with a tensor product of the set of shape functions in the spatial domain and stochastic functions spanning the stochastic space.

\subsection{Boundary roughness quantification}

Modification of the boundary of a physical domain can result in either of the following two scenarios: (i) the elements in contact with the boundary surface (having one or more nodes on the boundary) can have their edges modified following the movement of the nodes or (ii) a set of elements may be moved along with the boundary in addition to a modification in shape. The scenario of perturbation of one particular element in the domain is graphically depicted in Figure 2, while Figure 3 illustrates the realizations of a perturbed boundary region of a physical domain. Let us denote the deterministic domain within the 'red' box in Figure 3 as $\mathscr{D}_{\mathrm{bc}} \subset \mathscr{D}$ and the set of elements of the baseline model lying within this by the set $\Delta\left(\mathscr{D}_{\mathrm{bc}}\right)$. We then specify the stochastic set $\Delta\left(\mathscr{D}_{\theta}\right)$ consisting of the random realizations of the elements in $\Delta\left(\mathscr{D}_{\mathrm{bc}}\right)$. Figure 2 shows a sample realization of the configuration of an element in $\Delta\left(\mathscr{D}_{\theta}\right)$. A generic spatial domain meshed with quadrangular elements has been considered here. The mesh is assumed to be unstructured, and hence, the formulation can be trivially extended to the case of uniform mesh. The coordinate transformation from 


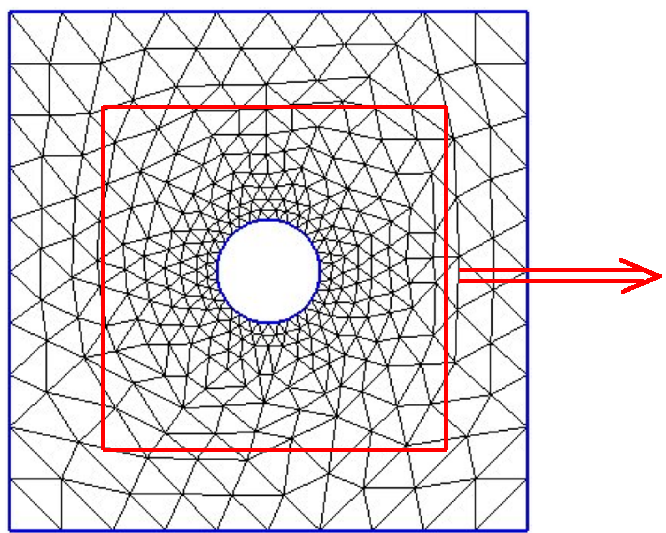

(a) Original configuration

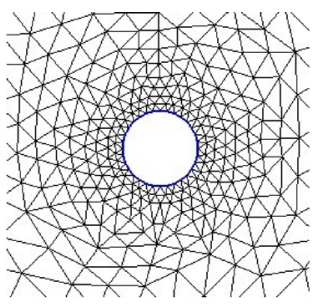

(b) Undeformed mesh

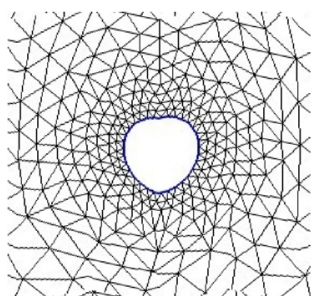

(d) Deformed mesh 2

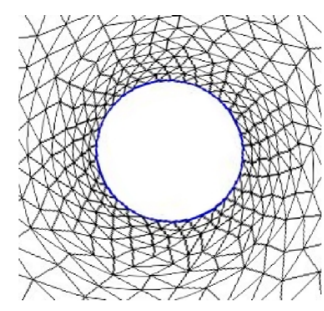

(c) Deformed mesh 1

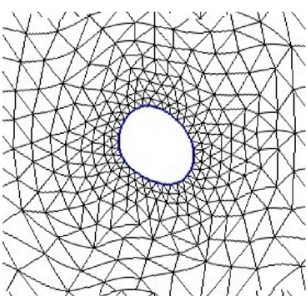

(e) Deformed mesh 3

Figure 3. The original meshed configuration of a plate with a hole at the center (a) and the realizations of the random geometrical deformation of the center hole. The refined mesh adjacent to the hole are shown before and after the random perturbation. The coarse mesh outside the marked rectangular region remains unchanged. (a) Original configuration. (b) Undeformed mesh. (c) Deformed mesh 1. (d) Deformed mesh 2.

(e) Deformed mesh 3.

the deterministic to master element is as given in Equation (34). The deterministic element in turn is transformed into the perturbed element $A^{\prime \prime} B^{\prime \prime} C^{\prime \prime} D^{\prime \prime}$ via the transformation

$$
\chi_{i}(\theta)=Q_{i}^{\theta}\left(\eta_{1}, \ldots, \eta_{d} ; \theta\right) \quad \forall i=1, \ldots, d ; \quad \text { and } \quad \Delta\left(\mathscr{D}_{\theta}\right)=\overline{\mathbf{Q}}^{\theta}\left(\Delta\left(\mathscr{D}_{\mathrm{bc}}\right)\right)
$$

where $\chi=\left\{\chi_{1}, \ldots, \chi_{d}\right\}$ is the set of independent coordinate directions specifying the points in the perturbed element and $\eta=\left\{\eta_{1}, \ldots, \eta_{d}\right\}$ denotes the same in the deterministic element domain.

The roughness of the boundary surface can be quantified with the correlation of the positional coordinates of the FE nodes of the elements in $\Delta\left(\mathscr{D}_{\theta}\right)$. The theoretical development of the discrete KL expansion, presented in Section 3.1, is utilized in this context to represent this boundary randomness using a denumerable set of random variables. Here, we use a covariance function $C\left(\mathbf{r}_{1}, \mathbf{r}_{2}\right)$ description of the input random field with the position coordinates $\left(\mathbf{r}_{1}, \mathbf{r}_{2}\right.$ on $\left.\mathscr{D}_{\mathrm{bc}}\right)$ of the nodes of the element in the baseline deterministic domain $\mathscr{D}_{\mathrm{bc}}$. Following this, a discrete spectral decomposition of $C$ gives $C \phi_{i}=\lambda_{i} \phi_{i}$ with $\mathbf{r}=\sum_{i=0}^{p_{M}} \phi_{i} \mathscr{H}_{i}(\xi), \mathbf{r} \in \mathbb{R} m$, where $\mathbf{r}$ is the vector of the position coordinates of the nodes lying on the random boundary (or in the boundary region $\mathscr{D}_{\mathrm{bc}}$ ) approximated with $M$ random variables with a $p_{M}$ th-order chaos expansion.

The parameterization of the element coordinates in the perturbed element in terms of the random variables $\left(\xi=\left\{\xi_{1}, \ldots, \xi_{M}\right\}\right)$ used to model the boundary roughness would lead to the transformed coordinate vector $\chi$ being expressed as $\chi=\langle\chi\rangle+\chi^{\prime}$, where $\langle\chi\rangle$ is the deterministic component while $\chi^{\prime}$ is the zero mean perturbation components. The objective here is to obtain a mapping between the coordinates of the master element $\eta$ and the samples of the perturbed configuration $\chi$.

Thus, we do not consider remeshing the domain for each random realization of its boundary. Only the perturbation of the coordinates of the nodes lying on the boundary region is incorporated into the formulation with a set of random variables. This is illustrated in Figure 3, which shows a typical geometrical configuration of a plate with a hole at the center. Here, the nodes lying inside the marked region are modeled with a set of random variables for the realization of the perturbed configurations, while those outside it remain unchanged. Hence, all the nodes in the region adjacent to the circle at the center of the plate are moved along with the boundary. The perturbation of these nodes can be modeled with the mapping described in Section 5.3. It might be mentioned here that the choice of 
the depth of the boundary region $\mathscr{D}_{\mathrm{bc}}$ from the random boundary designated in the baseline model is governed by the degree of boundary perturbation considered for a particular meshed domain. If the boundary perturbation is small such that the random realizations of the boundary can be captured wholly by the elements lying on the boundary such that only the nodes lying on the boundary has to be modified, then it is sufficient to consider $\mathscr{D}_{\mathrm{bc}}$ to be the boundary $\partial \mathscr{D}$ of the baseline model.

\subsection{Mapping the random domain to the master domain}

We denote the nodal coordinates of the deterministic element as the vector $\eta_{i_{j}}=\left\{\eta_{i_{1}}, \ldots\right.$, $\left.\eta_{i_{n_{e}}}\right\} \forall i=1, \ldots, d$. For the deterministic case, the mapping of the master element (which we denote by $\mathscr{D}_{M}$, say) to the deterministic element is given as $\eta_{i}=\sum_{k=0}^{n_{e}} \mathscr{N}_{k}^{p}(\tilde{\eta}) \eta_{i_{k}} \quad \forall i=$ $1, \ldots, d$, where $\mathscr{N}^{p}=\left\{\mathscr{N}_{1}^{p}, \ldots, \mathscr{N}_{n_{e}}^{p}\right\}$ are the $p$ th-order shape multidimensional Lagrange basis functions ( $n_{e}$ being the number of nodes per element). Hence, following Equation (43), we write that

$$
\eta_{i}=Q_{i}^{M}\left(\tilde{\eta}_{1}, \ldots, \tilde{\eta}_{d}\right) \quad \forall i=1, \ldots, d ; \quad \text { such that } \quad \Delta(\mathscr{D})=\overline{\mathbf{Q}}^{M}\left(\Delta\left(\mathscr{D}_{M}\right)\right)
$$

which gives the mapping from the deterministic to master element. Combining the preceding two equations, we have

$$
\Delta\left(\mathscr{D}_{\theta}\right)=\overline{\mathbf{Q}}^{\theta}\left(\overline{\mathbf{Q}}^{M}\left(\Delta\left(\mathscr{D}_{M}\right)\right)\right)
$$

Thus, the mapping from the master element to the perturbed element now involves a spatial as well as stochastic transformation. Hence, $\chi^{\theta}$, which denoted the coordinate directions for each sample realization of the random domain, is now expressed as a function of a denumerable set of random variables used to model the boundary perturbations and the coordinate directions of the master element; that is, $\chi(\tilde{\eta}, \xi(\theta))=\left\{\chi_{1}(\tilde{\eta}, \xi(\theta)), \ldots, \chi_{d}(\tilde{\eta}, \xi(\theta))\right\}$. As a result, the interpolation of the spatial coordinates within the random element is given as a tensor double dot product

$$
\chi_{i}(\tilde{\eta}, \xi)=\left[\mathscr{N}(\tilde{\eta}) \otimes \mathscr{N}_{\theta}(\xi)\right]:\left\{\chi_{i}^{j k}\right\}=\underline{\underline{\mathbf{N}}}(\tilde{\eta}, \theta): \underline{\underline{\chi}}_{i} \quad \forall i=1, \ldots, d
$$

where $\underline{\underline{\chi}}_{i}$ is a second-order tensor of the field $\chi_{i}$ at the nodal points in the tensor product space $\Delta(\mathscr{D}) \otimes \Theta^{(M)}$. If the matrix $\chi_{i}^{j k}$ is of dimension $n_{e} \times M$, where $n_{e}$ is the number of FE nodes associated with the element, then it suggests that the coefficient associated with a particular node has $M$ perturbation components. This suggests that the perturbed element can be described uniquely in a $d$-dimensional spatial domain and an $M$-dimensional stochastic domain.

It is obvious from Equation (46) that the tensor shape function $\underline{\underline{\mathbf{N}}}$ is stochastic in nature. To keep things simple, we consider the boundary roughness as the only source of uncertainty in the present case and ignore any effect of parametric or forcing randomness. The Jacobian $\mathbf{J}_{\theta}$ presented in the context of the discussion of Equations (41) and (42) can now be expressed as a function of the input random variables as $\mathbf{J}_{\tilde{\eta}}(\tilde{\eta}, \xi(\theta))=\partial \chi(\tilde{\eta}, \xi(\theta)) / \partial \tilde{\eta}$. The differential volume $\Delta\left(\mathscr{D}_{\theta}\right)$ in the random boundary can be transformed into the master element as

$$
d \mathscr{D}_{\theta}=d \chi_{i} \ldots d \chi_{d}, \quad \text { where } \quad d \chi_{i}=\frac{\partial \chi_{i}}{\partial \tilde{\eta}} d \tilde{\eta}
$$

following which, $\quad d\{\chi\}=\left[\mathbf{J}_{\tilde{\eta}}(\tilde{\eta}, \theta)\right] d\{\tilde{\eta}\}$

$$
\text { Hence, } \quad d \mathscr{D}_{\theta}=\operatorname{det}\left|\mathbf{J}_{\tilde{\eta}}\right| d \tilde{\eta}_{1} \ldots d \tilde{\eta}_{d}=\operatorname{det}\left|\mathbf{J}_{\tilde{\eta}}\right| d \mathscr{D}_{M}
$$

The differential operator $\nabla_{\chi^{\theta}}$ in Equation (41) is transformed under the coordinate mapping as

$$
\nabla_{\chi^{\theta}}=\left[\mathbf{J}_{\tilde{\eta}}(\tilde{\eta}, \xi(\theta))\right]^{-1} \nabla_{\tilde{\eta}}
$$


where $\nabla_{\tilde{\eta}}$ denotes the differential operator along the coordinate directions of the master element. For the implementation of the spectral Galerkin method, it is essential to have a representation of the stochastic quantities in terms of polynomials of the input random variables. This allows efficient computation of the expectation of these stochastic polynomial functions. To this end, the expression for the elements of the Jacobian matrices is presented here as

$$
\left(\mathbf{J}_{\tilde{\eta}}(\tilde{\eta}, \xi(\theta))\right)_{i j}=\left[\frac{\partial \mathscr{N}(\tilde{\eta})}{\partial \tilde{\eta}_{j}} \otimes \mathscr{N}_{\theta}(\xi)\right]: \underline{\underline{\chi}}_{i}
$$

Taking $\mathscr{N}_{\theta_{0}}=1$, the preceding Jacobian matrix $\mathbf{J}_{\tilde{\eta}}$ can be expressed as

$$
\mathbf{J}_{\tilde{\eta}}=\mathbf{J}_{\tilde{\eta}_{0}}+\sum_{i=1}^{M} \mathscr{N}_{\theta i}(\xi) \mathbf{J}_{\tilde{\eta}_{i}} \quad \text { where } \quad\left(\mathbf{J}_{\tilde{\eta}_{i}}\right)_{m n}=\sum_{k} \frac{\partial \mathscr{N}_{k}(\tilde{\eta})}{\partial \tilde{\eta}_{n}}\left(\underline{\underline{\chi}}_{m}\right)_{k i}
$$

It can be easily identified that $\mathbf{J}_{\tilde{\eta}_{0}}$ is the Jacobian associated with the transformation of the deterministic element into the parent element.

The inverse of the Jacobian matrix can be expressed with polynomial functions of the random variables using the Neumann-type series expansion as

$$
[\mathbf{J}(\tilde{\eta}, \xi(\theta))]^{-1}=\mathbf{J}_{\tilde{\eta} 0}^{-1} \sum_{k=0}^{p}(-1)^{k}\left(\mathbf{J}_{\tilde{\eta} 0}^{-1} \sum_{i} \mathscr{N}_{\theta_{i}}(\xi) \mathbf{J}_{\tilde{\eta} i}\right)^{k}=\sum_{k=0}^{p} \overline{\mathscr{H}}_{k}(\theta) \mathfrak{J}_{\tilde{\eta}_{k}}
$$

where $\overline{\mathscr{H}}_{k}$ is the stochastic polynomial function, which is obtained from combining the stochastic polynomials in the preceding equation. Of course, $\overline{\mathscr{H}}_{0}=1$ and $\mathfrak{J}_{\tilde{\eta}_{k}}$ are the corresponding product of the Jacobian matrices. The Neumann-type series expansion has been performed under the assumption that the spectral radius of the matrix $\mathbf{J}_{\tilde{\eta}_{0}}$ is larger compared with the $\mathbf{J}_{\tilde{\eta}_{i}}$ components. This assumption is valid considering the fact that the major change in the elemental volume is captured in the transformation of the deterministic element into the master element (which is $A^{\prime} B^{\prime} C^{\prime} D^{\prime} \rightarrow A B C D$ in Figure 2), while transformation from the deterministic to stochastic element is small under the assumption of small random perturbation of boundary topology. Taking this into account, it is possible to capture the transformation with a moderately low degree of Neumann expansion.

In case the assumption regarding the spectral radius of $\mathbf{J}_{\tilde{\eta}_{0}}$ does not hold, the inverse can be evaluated from the solution of the equation $\mathbf{J}_{\tilde{\eta}} \mathbf{J}_{\tilde{\eta}}^{-1}=\mathbf{I}$. We assume that the inverse of the Jacobian matrix can be expressed as a matrix series such that $\mathbf{J}_{\tilde{\eta}}^{-1}=\sum_{k=0}^{p} \mathscr{H}_{k}(\theta) \mathfrak{J}_{\tilde{\eta}_{k}}$, where $\mathfrak{J}_{\tilde{\eta}_{k}}$ are the undetermined matrix coefficients and $\mathscr{H}_{k}(\theta)$ are the multidimensional orthogonal polynomials spanning the stochastic Hilbert space. These can be evaluated if we apply a Galerkin orthogonalization of the residual with respect to the orthogonal stochastic basis functions as

$$
\begin{gathered}
\left\langle\mathscr{H}_{i}(\theta), \mathbf{J}_{\tilde{\eta}}\left(\sum_{k=0}^{p} \mathscr{H}_{k}(\theta) \mathfrak{J}_{\tilde{\eta}_{k}}\right)\right\rangle_{L^{2}\left(\Theta^{(M)}\right)}=\left\langle\mathscr{H}_{i}(\theta), \mathbf{I}\right\rangle_{L^{2}\left(\Theta^{(M)}\right)} \quad \forall i=0, \ldots, p \\
\text { from which, } \quad\left[\begin{array}{c}
\overline{\mathbf{J}}_{\tilde{\eta}}^{b} \\
\end{array}\right]\left[\begin{array}{c}
{\left[\mathfrak{J}_{\tilde{\eta}_{0}}\right]} \\
\vdots \\
{\left[\mathfrak{J}_{\tilde{\eta}_{p}}\right]}
\end{array}\right]=\left[\begin{array}{c}
\mathbf{I} \\
\vdots \\
0
\end{array}\right]
\end{gathered}
$$

where $\overline{\mathbf{J}}_{\tilde{\eta}}^{b}$ is the coefficient matrix composed of blocks of the Jacobian matrix series from Equation (50) such that the $i, j$ block of the matrix is obtained as $\left[\overline{\mathbf{J}}_{\tilde{\eta}}^{b}\right]_{i, j}=$ $\left\langle\mathscr{H}_{i}(\theta) \mathbf{J}_{\tilde{\eta}}(\theta) \mathscr{H}_{j}(\theta)\right\rangle_{L^{2}\left(\Theta^{(M)}\right)}$ and $\langle\cdot\rangle_{L^{2}\left(\Theta^{(M)}\right)}$ denotes the inner product in the $L^{2}$ stochastic Hilbert space. Hence, we have to solve for the block matrices $\mathfrak{J}_{\tilde{\eta}_{k}}$ from Equation (53) for each element 
that is included within the perturbed boundary region. It might be noted that the solution of the preceding matrix equation is trivially parallelizable because the column vectors of all the $\mathfrak{J}_{\tilde{\eta}_{k}}$ can be solved in parallel. This method is more expensive than the Neumann expansion method given in Equation (51), but it gives a good approximation of the inverse Jacobian. Thus, in the present development, we would consider that the inverse Jacobian matrix is approximated as a matrix series $\mathbf{J}_{\tilde{\eta}}^{-1}=\sum_{k=0}^{p} \overline{\mathscr{H}}_{k}(\theta) \mathfrak{J}_{\tilde{\eta}_{k}}$, where $\overline{\mathscr{H}}_{k}(\theta)$ are the stochastic polynomials obtained from the Neumann expansion or the stochastic Galerkin method and $\mathfrak{J}_{\tilde{\eta}_{k}}$ are the corresponding matrix coefficients.

Recalling Equation (48), the transformation of the differential operator can be rewritten as

$$
\nabla_{\chi^{\theta}}=\left(\sum_{k=0}^{p} \overline{\mathscr{H}}_{k}(\theta) \mathfrak{J}_{\tilde{\eta}_{k}}\right) \nabla_{\tilde{\eta}}
$$

following from Equation (51). We note that the Jacobian matrix is expressed as a sum of Jacobian matrices weighted with stochastic polynomials as in Equation (50). The evaluation of the determinant of this random Jacobian matrix, as required in Equation (47), can be written explicitly in terms of a set of polynomial random functions as

$$
\operatorname{det}\left|\mathbf{J}_{\tilde{\eta}}\right|=\sum_{i} \mathscr{H}_{i}^{\operatorname{det}}(\theta) \operatorname{det}\left|\mathbf{J}_{\tilde{\eta}_{i}}^{\pi}\right| \quad \text { with } \quad \mathscr{H}_{0}^{\text {det }}=1
$$

where $\mathscr{H}_{\tilde{\eta}_{i}}^{\text {det }}$ is the stochastic polynomial associated with the Jacobian $\mathbf{J}_{\tilde{\eta}}$. These polynomials are obtained by expanding the determinant of a full rank Jacobian matrix, and the det $\left|\mathbf{J}_{\tilde{\eta}_{i}}^{\pi}\right|$ are obtained as the combination of the Jacobian matrix with appropriate rearrangement of rows as per the coefficient random polynomial function. The detailed derivation to explicitly express the determinant of the sum of the series of the form $\operatorname{det}\left|\mathbf{J}_{0}+\sum_{i} \alpha_{i} \mathbf{J}_{i}\right|$ with polynomial functions of the scalar coefficient $\alpha_{i}$ is provided in Appendix B. The maximum degree of the polynomial in $\alpha_{i}$ is governed by the rank of the matrices $\mathbf{J}_{i}$.

The expression of the element-level system matrices, which are perturbed by random boundary fluctuations, would involve products of random polynomial functions $\overline{\mathscr{H}}_{i}(\theta)$ and $\mathscr{H}_{j}^{\operatorname{det}}(\theta)$ obtained from Equations (51) and (55). We introduce the notation $\mathscr{H}_{k}^{S}(\theta)=\overline{\mathscr{H}}_{i} \overline{\mathscr{H}}_{j} \mathscr{H}_{l}^{\text {det }}$, where $k$ is the cardinality of the set $\mathscr{J} \mathscr{H}$, which consists of an ordered set of the stochastic product functions $\mathscr{J}_{\mathscr{H}}=\left\{\mathscr{H}_{k}^{S}(\theta)=\mathscr{\mathscr { H }}_{i} \mathscr{\mathscr { H }}_{j} \mathscr{H}_{l}^{\text {det }} \forall i, j, l\right\}$.

We discuss the relationship of the proposed methodology to the stochastic mapping technique implemented using the concept of boundary-conforming coordinate system introduced in [12]. The latter utilizes the solution of Laplace equations to obtain the stochastic mapping of the random element boundaries to the deterministic domain. This concept relies on the assumption that the random boundary is realized with stochastic mapping of the structured body-fitted curvilinear coordinates. Thus, the regularity requirements on the boundary applies to this case and in general the random mapping. In contrast, the proposed method is applicable to different types of physical domains $\mathscr{D} \subset$ $\mathbb{R}^{d}$, where the information of the positional coordinates of the set of FE nodes lying on the boundary or a region adjacent to the boundary of the baseline model (usually the deterministic ideal domain) is the input to the stochastic model along with a covariance function describing the correlation of the nodal coordinates across the boundary region. For example, if the fluctuation of a portion of the domain boundary is described with body-fitted curvilinear coordinates $\overline{\mathbf{r}} \in \mathscr{D}$, then it is a simple exercise to obtain the coordinate transformation matrix $\mathbf{T}$ (and its inverse) such that $\mathbf{x}=\mathbf{T} \overline{\mathbf{r}}$ where $\mathbf{x}$ is the global Cartesian axes. The covariance function described with $C_{a}\left(\overline{\mathbf{r}}_{1}, \overline{\mathbf{r}}_{2}\right)=f\left(\Delta \overline{\mathbf{r}} / L_{\overline{\mathbf{r}}_{c}}\right)$ is transformed to the Cartesian system as $C_{a}\left(\mathbf{x}_{1}, \mathbf{x}_{2}\right)=f\left(\Delta\left\{\mathbf{T}^{-1} \mathbf{x}\right\} / L_{\mathbf{x}_{c}}\right)$, where $L_{\mathbf{x}_{c}}$ is the transformed correlation lengths along the Cartesian axes. The proposed method relies on the assumption that the boundary perturbations and, in general, changes to the topology of the physical domain can be captured with perturbation of the elemental domains as shown in Figure 3. However, this method would not be computationally meaningful in case of significant changes in the shape of the physical domain because the solution accuracy that would be obtained by transforming the mesh 
of the original domain may be unacceptable. Remeshing the domain, in such cases, would receive serious consideration. We have analyzed the accuracy of the solution obtained with the proposed method with respect to a benchmark brute-force Monte Carlo simulation, for a given degree of input perturbation, in the results section later in this article.

\subsection{Solution in tensor product space}

The test functions for the weak formulation of the diffusion problem defined on a random domain would consist of all those functions that exist in the tensor product approximation space of the spatial basis functions and the stochastic polynomials. The solution is thus approximated within the element domain as

$$
u_{e}=\left[\mathscr{N}^{p}(\tilde{\eta}) \otimes \mathscr{H}^{q, M}(\theta)\right]: \underline{\underline{u}}_{p}^{q, M}=\underline{\underline{N H}}(\tilde{\eta}, \theta): \underline{\underline{u}}_{p}^{q, M}=\sum_{i=1}^{n_{e}} \sum_{j \in \mathscr{I}_{m}} \mathscr{N}_{i}^{p}(\tilde{\eta}) \mathscr{H}_{j}^{q, M}(\theta) u_{i j}
$$

where $\mathscr{N}^{p}(\tilde{\eta})$ denotes the $p$ th-order spatial basis functions in the master domain and $\mathscr{H}^{q, M}$ are the $q$ th-order orthogonal stochastic polynomials spanning the $M$-dimensional input stochastic space.

Thus, the weak form of the diffusion equation defined on a domain with random boundary in Equation (40) can be rewritten with these stochastic polynomial coefficient functions using Equations (54)-(56) as

$$
\begin{aligned}
\mathscr{B}_{\Delta\left(\mathscr{D}_{M}\right) \otimes \Theta^{(M)}}\left(u_{e}, v_{e}\right)= & \int_{\Theta^{(M)}} \sum_{i \in \mathscr{J}_{\mathscr{H}}} \mathscr{H}_{i}^{S}(\theta) \int_{\Delta\left(\mathscr{D}_{M}\right)} K_{e}\left(\mathfrak{J}_{\tilde{\eta}_{k}} \nabla_{\tilde{\eta}} \underline{\underline{N H}}: \underline{\underline{v}}_{p}^{q, M}\right) \\
& \cdot\left(\mathfrak{J}_{\tilde{\eta}_{j}} \nabla_{\tilde{\eta}} \underline{\underline{N H}}: \underline{\underline{u}}_{p}^{q, M}\right) \operatorname{det}\left|\mathbf{J}_{\tilde{\eta}_{l}}^{\tilde{\pi}}\right| d \tilde{\eta} d P_{\xi}(\theta) \\
\mathscr{L}_{\Delta\left(\mathscr{D}_{M}\right) \otimes \Theta^{(M)}}\left(v_{e}\right)= & \int_{\Theta^{(M)}} \sum_{j} \mathscr{H}_{\tilde{\eta} \xi_{j}}^{\operatorname{det}}(\theta) \int_{\Delta\left(\mathscr{D}_{M}\right)}\left(\underline{\underline{N H}}: \underline{\underline{v}}_{p}^{q, M}\right) Q\left(\mathbf{r}_{h}\right) \operatorname{det}\left|\mathbf{J}_{\tilde{\eta} \xi_{j}}^{\pi}\right| d \tilde{\eta} d P_{\xi}(\theta)
\end{aligned}
$$

with $\quad \nabla_{\tilde{\eta}} \underline{\underline{N H}}: \underline{\underline{u}}_{p}^{q, M}=\left(\left[\left(\nabla_{\tilde{\eta}} \mathscr{N}^{p}(\tilde{\eta})\right) \otimes \mathscr{H}^{q, M}(\theta)\right]\right): \underline{\underline{u}}_{p}^{q, M}$

where $d P_{\xi}(\theta)$ is the joint probability measure of the vector of random variables $\xi$. The term $\mathscr{H}_{k}^{S}(\theta)$ is a compact notation of the product of stochastic polynomial functions as $\mathscr{H}_{n}^{S}=\overline{\mathscr{H}}_{i} \overline{\mathscr{H}}_{j} \mathscr{H}_{\tilde{\eta} \xi}$ det . The bilinear operator in the previous equation necessitates the evaluation of expectation of polynomials of random variables of the form $\mathbb{E}\left[\mathscr{H}_{k}^{S} \mathscr{H}_{i}^{q, M} \mathscr{H}_{j}^{q, M}\right]$. These are pre-calculated and plugged into the FE code during the assembly of the large FE system matrices. Alternatively, quadrature-based integration schemes (such as the Gauss-Hermite quadrature) can be used to numerically evaluate the integrations in the stochastic space. However, for high-dimensional stochastic problems, this integration can have significant computational overhead (as demonstrated in Table I).

Table I. Computational time (in seconds) to obtain the system matrix A with multidimensional Gauss-Hermite quadrature points for the integration in stochastic space.

\begin{tabular}{lccccc}
\hline No. of quad points & PC order 2 & PC order 4 & PC order 6 & PC order 8 & PC order 10 \\
\hline 5 & 0.4 & 7 & & & \\
6 & 0.7 & 13 & & & \\
8 & 2.2 & 41 & 374 & & \\
10 & 5.1 & 103 & 867 & 4319 & 36,797 \\
12 & 11 & 216 & 1817 & 9301 & 3 \\
\hline
\end{tabular}

The random boundary fluctuation has been represented with four independent Gaussian random variables in all the cases. The number of the Gauss-Hermite quadrature points used along each stochastic dimension is shown in the leftmost column, and it is seen that the computational time increases significantly with the order and the number of quadrature points. 
Equation (57) leads to a system of linear equations $\mathbf{A U}=\mathbf{F}$ of dimension $n_{\mathrm{PC}} \times n_{\text {dof }}$, where $n_{\mathrm{PC}}$ is the number of terms of the Polynomial Chaos (PC) expansion given by the formula $n_{\mathrm{PC}}=$ $\left(\begin{array}{c}M+q \\ M\end{array}\right)$, where $M$ is the number of random variables and $q$ is the order of chaos expansion. Each element-level matrix that is assembled to obtain the global system matrix $\mathbf{A}$ is obtained as

$$
\begin{aligned}
\left(\mathbf{A}_{K, L}^{e}\right)_{I J}= & \sum_{k \in \mathscr{J} \mathscr{H}} \mathbb{E}\left[\mathscr{H}_{k}^{S} \mathscr{H}_{K}^{q, M} \mathscr{H}_{L}^{q, M}\right] \int_{\Delta\left(\mathscr{D}_{M}\right)} K_{e} \sum_{g=1}^{d}\left(\sum_{h=1}^{d}\left(\mathfrak{J}_{\tilde{\eta}_{k}}\right)_{g h} \nabla_{\tilde{\eta}_{h}} \mathscr{N}_{I}^{p}\right) \\
& \cdot\left(\sum_{l=1}^{d}\left(\mathfrak{J}_{\tilde{\eta}_{k}}\right)_{g l} \nabla_{\tilde{\eta}_{l}} \mathscr{N}_{J}^{p}\right) \operatorname{det}\left|\mathbf{J}_{\tilde{\eta}_{k}}^{\pi}\right| d \tilde{\eta} d P_{\xi}(\theta)
\end{aligned}
$$

We present here an analysis of the computational complexity for the evaluation of the terms of the system matrix A. If we assume that an $n_{\mathrm{GQ}}$ point Gauss-Legendre quadrature is utilized to perform the integration in the spatial domain and that the evaluation of the terms of the matrix in Equation (58) is given by $n_{s}$, then the evaluation of the system at each point in the stochastic space is given by $n_{\mathrm{GQ}}^{d} n_{s}$, where $d$ is the number of dimensions in the spatial domain. If $n_{p e}$-noded elements are used, then the total cost of evaluating an element-level matrix at one specific point in the stochastic space is given by $n_{p e}^{2} n_{\mathrm{GQ}}^{d} n_{s}$. If the random field is approximated with $n_{k}$ stochastic functions, that is, $\mathscr{H}_{k}^{S}$ has $n_{k}$ terms, and if the expectation terms associated with each system-level term is pre-calculated, then each block of the matrix $\mathbf{A}_{K, L}^{e}$ in Equation (58) is calculated with $n_{k} n_{p e}^{2} n_{\mathrm{GQ}}^{d} n_{s}$ operations. However, if a quadrature scheme is implemented in order to evaluate the stochastic integration operations during the evaluation of the system matrices, then the cost can be calculated as follows. If we assume that an $n_{q}$ point quadrature scheme is chosen as per the weighting functions (i.e., Gauss-Hermite quadrature for Gaussian random variables or Gauss-Legendre quadrature for uniform random variables), then the cost is given as $n_{q}^{M}\left(n_{p e}^{2} n_{\mathrm{GQ}}^{d} n_{s}\right)$, where $M$ is the number of random variables. As a result, precomputing the expectation operators is going to be advantageous in most cases because $n_{q}^{M}$ would almost always be greater than $n_{k}$. The value of $n_{k}$ is governed by the chosen chaos order and the dimension of the approximating stochastic functions. Hence, to evaluate the system matrix with one element using a $q$ th-order chaos expansion in $M$-dimensional stochastic space, we have $n_{\mathrm{PC}}^{2} n_{q}^{M}\left(n_{p e}^{2} n_{\mathrm{GQ}}^{d} n_{s}\right)$. Thus, the order of computational complexity grows as $\mathscr{O}\left(n_{\mathrm{PC}}^{2} n_{q}^{M}\right)$ with the stochastic dimension. Now, a $q$-point quadrature rule exactly integrates a function of order $\leqslant 2 q-1$. Hence, the number of quadrature points must increase with the order of chaos expansion.

Table I gives the computational time to evaluate the element-level system matrix using the GaussHermite quadrature points as presented in the preceding paragraph. The calculations have been performed on eight computational cores of identical capability with multithreaded FORTRAN 90 subroutines using gfortran compilers. It has been seen that we require at least one more quadrature point than the order of chaos expansion to obtain a good approximation of the stochastic integration (hence, the boxes corresponding to high-order chaos expansion with fewer quadrature points in Table I have been left blank). We see that the calculations approximately match the computational complexity orders calculated in the previous paragraph.

Recalling the classification of parameter-based approximation of the field variable and the element geometry in FE analysis [48], the isoparametric formulation is one in which the degree of interpolation $(p)$ functions used to approximate the element geometry $\left(G_{p}\right)$ and the unknown field $\left(F_{p}\right)$ is the same; that is, $G_{p}=F_{p}$. Here, the stochastic response $\underline{\underline{u}}_{p}^{q, M}$ has been approximated with $p$ th-order spatial interpolation functions, which is identical to that for the spatial geometrical approximation. However, the order of stochastic approximation $q$ would be generally larger (or at best equal) to that used to approximate the random boundary. This implies that this is a subparametric formulation with respect to the stochastic approximation.

The transformation of the boundary integral terms for the Neumann part of the boundary $\Gamma_{\theta}^{1}$ where a non-zero flux is imposed (as given in Equation (40) is discussed here. This term is written as a boundary integral on the element (which lies on the perturbed geometry): 


$$
\begin{aligned}
\left.\int_{\Theta(M)} \oint_{\partial \Delta\left(\mathscr{D}_{\theta}\right)} \mathbf{n} \cdot \nabla u\right|_{\partial \Omega_{\theta}} \underline{\underline{N H}} d \Omega_{\theta} d P_{\xi}(\theta) & =\int_{\Theta^{(M)}} \oint_{\partial \Delta\left(\mathscr{D}_{\theta}\right)} q_{c} \underline{\underline{N H}} d \Omega_{\theta} d P_{\xi}(\theta) \\
& =\int_{\Theta^{(M)}} \oint_{\partial \Delta\left(\mathscr{D}_{M}\right)} q_{c} \underline{\underline{N H}}\left(\left.\tilde{\eta}\right|_{\partial \Omega}, \xi\right) J_{\partial \Omega_{\theta}} d \Omega d P_{\xi}(\theta)
\end{aligned}
$$

In the preceding equation, $J_{\partial \Omega_{\theta}}$ denotes the stochastic Jacobian transformation of the elemental boundary into the master boundary, and $\mathrm{NH}$ is the stochastic shape functions introduced earlier in this section. All the earlier discussion on the Jacobian transformation matrix for the transformation of the elemental random volume into its parent domain is also applicable here. The boundary flux term is imposed on every stochastic sample realization, and hence, any functional dependence of the flux on the boundary coordinates is transformed to the master domain using the isoparametric transformation introduced in Equation (46). The Dirichlet boundary condition is imposed in the strict sense where $u=G_{c}$ on $\Gamma_{\theta}^{0}$ for all $\theta \in \Theta$. Thus, with the isoparametric form, the imposition of the boundary condition follows intuitively from the previous theoretical development without additional mathematical complexity.

The proposed methodology enables the resolution of SPDEs defined on domains with random boundary topology within the framework of stochastic spectral Galerkin methods. The solutions expressed with orthogonal polynomials from the finite-dimensional stochastic Hilbert space require the solution of large block-sparse linear systems. This can be carried out with iterative Krylov solvers, as discussed in the following sections.

\section{UNIFIED TREATMENT OF PARAMETRIC AND BOUNDARY RANDOMNESS}

Here, we present a unified treatment of the parametric and boundary uncertainty of the random field using the methodologies presented in Sections 4 and 5. Assume that the parameter $K$ in Equation (40) is random such that $K=K(\xi(\theta))$, where $\theta \in \Theta^{M_{1}}$ is an $M_{1}$-dimensional stochastic space for the parametric randomness. We utilize the boundary uncertainty description presented in the previous section where the solution is approximated in an $M_{2}$-dimensional stochastic space $\Theta^{M_{2}}$. We assume that the random fields are stationary and square integrable. This gives the bilinear and linear forms, which are used as a starting point for the stochastic weak formulation on the domain with random boundary.

$$
\begin{aligned}
& \mathscr{B}_{\Delta\left(\mathscr{D}_{M}\right) \otimes \Theta^{(\bar{M})}}\left(u_{e}, v_{e}\right)=\int_{\Theta^{(\bar{M})}} \sum_{i \in \mathscr{I}_{q_{1}, M_{1}}} \mathscr{H}_{i}^{S}(\theta) \int_{\Delta\left(\mathscr{D}_{M}\right)}\left(\sum_{k \in \mathscr{I}_{q_{2}, M_{2}}} \mathscr{H}^{q_{2}, M_{2}}(\theta) K_{i}^{e}\right) \\
& \cdot\left(\mathfrak{J}_{\tilde{\eta}_{k}} \nabla_{\tilde{\eta}} \underline{\underline{N H}}: \underline{\underline{v}} \underline{\bar{Q}, \bar{M}}\right)\left(\mathfrak{J}_{\tilde{\eta}_{j}} \nabla_{\tilde{\eta}} \underline{\underline{N H}}: \underline{\underline{u}}_{p}^{\bar{Q}, \bar{M}}\right) \operatorname{det}\left|\mathbf{J}_{\tilde{\eta}_{l}}^{\tilde{N}}\right| d \tilde{\eta} d P_{\xi_{M_{1}}} d P_{\xi_{M_{2}}} \\
& \mathscr{L}_{\Delta\left(\mathscr{D}_{M}\right) \otimes \Theta^{(\bar{M})}}\left(v_{e}\right)=\int_{\Theta^{(\bar{M})}} \sum_{j} \mathscr{H}_{\tilde{\eta} \xi_{j}}^{\operatorname{det}}(\theta) \int_{\Delta\left(\mathscr{D}_{M}\right)}(\underline{\underline{N H}}: \underline{\underline{v}}=\underline{\bar{Q}, \bar{M}}) Q\left(\mathbf{r}_{h}\right) \operatorname{det}\left|\mathbf{J}_{\tilde{\eta} \xi_{j}}^{\pi}\right| d \tilde{\eta} d P_{\xi_{M_{1}}} d P_{\xi_{M_{2}}} \\
& \text { where } \quad \underline{\underline{N H}}: \underline{\underline{u}}^{\bar{Q}, \bar{M}}=\left[\mathscr{N}^{p}(\tilde{\eta}) \otimes \mathscr{H}^{\bar{Q}, \bar{M}}\left(\xi_{M_{1}}, \xi_{M_{2}}\right)\right]: \underline{\underline{u}}^{\overline{\mathrm{Q}}, \bar{M}}
\end{aligned}
$$

In the preceding equation, $\mathscr{H}^{\bar{Q}, \bar{M}}\left(\xi_{M_{1}}, \xi_{M_{2}}\right)$ are the orthogonal polynomials constructed in the stochastic tensor product space $\Theta^{\left(M_{1}\right)} \times \Theta^{\left(M_{2}\right)}$ whose dimension is $M_{1} \cup M_{2}$. Because the samples from $\Theta^{\left(M_{1}\right)}$ and $\Theta^{\left(M_{2}\right)}$ are taken to be independent of each other, the joint distribution is given by $d P_{\xi_{M_{1}}} d P_{\xi_{M_{2}}}$. The order of chaos expansion is chosen to be $\bar{Q}$, which is chosen based on the sensitivity of the response to each stochastic dimension. Normally, dimensionally adaptive stochastic functions can produce optimal convergence of the stochastic solution with fewer basis functions. [49-51]. 
The expression of the system matrix from Equation (60) can be written as

$$
\begin{aligned}
\left(\mathbf{A}_{K, L}^{e}\right)_{I J}= & \sum_{k \in \mathscr{J}_{H}} \mathbb{E}\left[\left(\mathscr{H}^{S} \mathscr{H}^{q_{2}, M_{2}}\right)_{k} \mathscr{H}_{K}^{\bar{Q}, \bar{M}} \mathscr{H}_{L}^{\bar{Q}, \bar{M}}\right] \int_{\Delta\left(\mathscr{D}_{M}\right)} K_{k}^{e} \sum_{g=1}^{d}\left(\sum_{h=1}^{d}\left(\mathfrak{J}_{\tilde{\eta}_{k}}\right)_{g h} \nabla_{\tilde{\eta}_{h}} \mathscr{N}_{I}^{p}\right) \\
& \left(\sum_{l=1}^{d}\left(\mathfrak{J}_{\tilde{\eta}_{k}}\right)_{g l} \nabla_{\tilde{\eta}_{l}} \mathscr{N}_{J}^{p}\right) \operatorname{det}\left|\mathbf{J}_{\tilde{\eta}_{k}}^{\pi}\right| d \tilde{\eta} d P_{\xi_{M_{1}}} d P_{\xi_{M_{2}}}
\end{aligned}
$$

where $\mathscr{I}_{H}$ is the cardinality of the set $H=\left\{\mathscr{H}_{i}^{S} \mathscr{H}_{j}^{q_{2}, M_{2}}\right.$ for all $\mathrm{i} \in \mathscr{I}_{q_{1}, M_{1}}$ and $\left.\mathrm{j} \in \mathscr{I}_{q_{2}, M_{2}}\right\}$. This leads to the linear system of equations of the form $\mathbf{A U}=\mathbf{F}$, where the dimension of system depends on the total number of random variables $\bar{M}$ and the order of chaos expansion $\bar{Q}$ chosen for the solution. The expectations $\mathbb{E}[\cdot]$ of the stochastic polynomials have to be precomputed and plugged in the solver for the efficient evaluation of the linear system. This gives a unified framework within which the diffusion problem with parametric uncertainty can be tackled on a domain that has random boundary fluctuations.

\section{RESULTS}

In this section, we present the results obtained from the numerical experiments performed with the methodologies proposed in the previous sections. The results for the discrete spectral decomposition of the covariance kernel is presented in Section 7.1. A comparison of this method with Galerkin's method of obtaining the KL eigen-components, as presented in Section 3.2, is also given in Section 7.1 to demonstrate the accuracy of the proposed method. Following this, the steady-state diffusion system with parametric uncertainty has been analyzed in Section 7.2.1 where FE shape functions have been utilized to interpolate the discrete random field within each element domain. Section 7.2.2 presents the unsteady response of a dynamic diffusion system on a domain with random boundary fluctuations, and its comparison with direct Monte Carlo simulation solution has been given.

\subsection{Discrete random-field representation}

In this section, we present the approximation errors associated with the proposed 'discrete KL expansion' discussed in Section 3.1. Here, we consider a lognormal random field that has been approximated with finite-order Hermite polynomials constructed with a set of Gaussian i.i.d. random variables. We study the convergence of the lognormal field with various parameters such as the input stochastic dimension, order of Hermite polynomials, and mesh parameter size in this section. We define an error metric as

$$
\varepsilon=\frac{\left\|C_{a}^{\text {exact }}-C_{a}^{\text {approx }}\right\|_{\mathrm{F}}}{\left\|C_{a}^{\text {exact }}\right\|_{\mathrm{F}}}
$$

Here, $C_{a}^{\text {exact }}$ is the exact covariance function of the random field at the Gauss integration points in the spatial domain. The approximated covariance kernel $C_{a}^{\text {approx }}$, synthesized from the discrete random field at the Gauss points, has been utilized to study the approximation error. Thus, $\varepsilon$ gives the accuracy of the approximate covariance function to the target kernel [52]. Here, $\varepsilon$ is an estimate of the error norm on the entire domain when considering the full covariance matrix or as cell values computed within each element of the FE mesh.

Here, the exact covariance function has been synthesized in two different ways to highlight the convergence of the approximate random field with respect to various approximating parameters. One method uses the exact covariance matrix from the original description of the covariance function over the entire physical domain at the discrete nodal locations. We have used an exponential covariance function for this purpose as $C_{a}^{\text {exact }}=\exp \left(-\left\|\mathbf{r}_{1}-\mathbf{r}_{2}\right\| / \mathbf{L}_{r}\right)$, where $\mathbf{L}_{r}$ is the correlation length of the underlying Gaussian random field. Figure 4(a) highlights the improvement in 


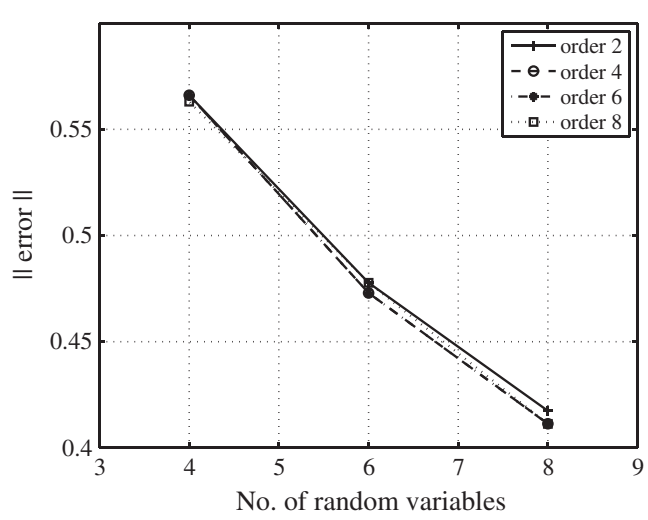

(a) Convergence of the error with inputs to chastic space dimension.

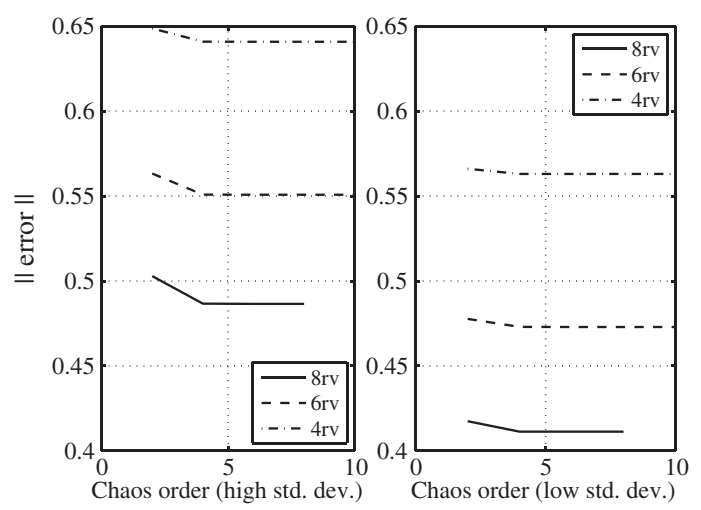

(b) Convergence of the error with chaos order.

Figure 4. Convergence of the Frobenius error norm of the covariance matrix with the order of chaos expansion and the input stochastic dimension. The values of input standard deviation are $\{0.10,0.20\}$ (low and high, respectively). (a) Convergence of the error with input stochastic space dimension. (b) Convergence of the error with chaos order.

accuracy with increasing dimension of the input stochastic field and also shows the effect of the order of chaos expansion on the stochastic field. Figure 4(b) shows the effect of the input variability of the stochastic field. It demonstrates that optimal convergence of the approximate random field is obtained by simultaneously controlling the dimension and the order of chaos such that the highest gradient of the error curve is obtained.

We construct $C_{a}^{M \text {,exact }} \in \mathbb{R}^{n \times n}$, which is the exact covariance kernel of the lognormal random field represented with $M$ i.i.d. Gaussian random variables; that is, the covariance matrix is $C_{a}^{M \text {,exact }}=\mathbb{E}\left[\left\{\exp \left(\overline{\mathbf{a}}_{0}+\sum_{i=1}^{M} \xi_{i} \tilde{\mathbf{a}}_{i}\right)-\mu_{\exp }\right\}^{T}\left\{\exp \left(\overline{\mathbf{a}}_{0}+\sum_{i=1}^{M} \xi_{i} \tilde{\mathbf{a}}_{i}\right)-\mu_{\exp }\right\}\right]$, where $\mu_{\exp }=$ $\mathbb{E}\left[\exp \left(\overline{\mathbf{a}}_{0}+\sum_{i=1}^{M} \xi_{i} \tilde{\mathbf{a}}_{i}\right)\right]$ is the mean of the exponential field. Using simple algebraic manipulations and simplifying these expressions for each $(p, q)$ th element of the matrix $C_{a}^{M \text {,exact }}$, we have

$$
\left[C_{a}^{M, \text { exact }}\right]_{p, q}=\exp \left(\left[\overline{\mathbf{a}}_{0}\right]_{p}+\left[\overline{\mathbf{a}}_{0}\right]_{q}\right)\left[\exp \left(\sum_{i=1}^{M} \frac{1}{2}\left(\left[\tilde{\mathbf{a}}_{i}\right]_{p}+\left[\tilde{\mathbf{a}}_{i}\right]_{q}\right)^{2}\right)-\exp \left(\sum_{i=1}^{M} \frac{1}{2}\left(\left[\tilde{\mathbf{a}}_{i}\right]_{p}^{2}+\left[\tilde{\mathbf{a}}_{i}\right]_{q}^{2}\right)\right)\right]
$$

Here, $\left[\overline{\mathbf{a}}_{0}\right]_{p}$ and $\left[\tilde{\mathbf{a}}_{i}\right]_{p}$ denote the $p$ th component of the deterministic and perturbation parts of the random-field vector. $M$ is the dimension of the stochastic space. Hence, the error constructed with this 'exact' covariance matrix using Equation (62) would highlight the approximation accuracy obtained with successive $p$-refinements of the stochastic space. Figure 5 gives the convergence of the lognormal random field with respect to the chaos order for different degrees of input variability of the input random field. The different dimensions $(4,6,8)$ of the input stochastic space have been considered. It shows that higher-order chaos functions have to be utilized to obtain the desired accuracy levels for higher input standard deviation.

Figure 6 gives the error in the covariance kernel in each element of the FE mesh for different dimensions of the input stochastic space and mesh parameter sizes. The exact and synthesized covariance kernels have been obtained at the Gauss quadrature points inside each element, and the Frobenius norm of these matrices gives the approximation error inside each elemental domain. The figures show that as the mesh is refined (or as $h_{\min }$ is reduced), the order of accuracy improves appreciably. Also, because the mesh has been deliberately taken to be unstructured, the particular elements in the mesh that are larger and more skewed have a higher value of error. Also, the improvement in the accuracy with the stochastic dimension (from 4 in Figure 6(a-c) to 200 in Figure 6(d-f)) is highlighted. This color map shows that as the mesh is refined, the 'blue' patches increase, signifying an improved approximation of the covariance function. 


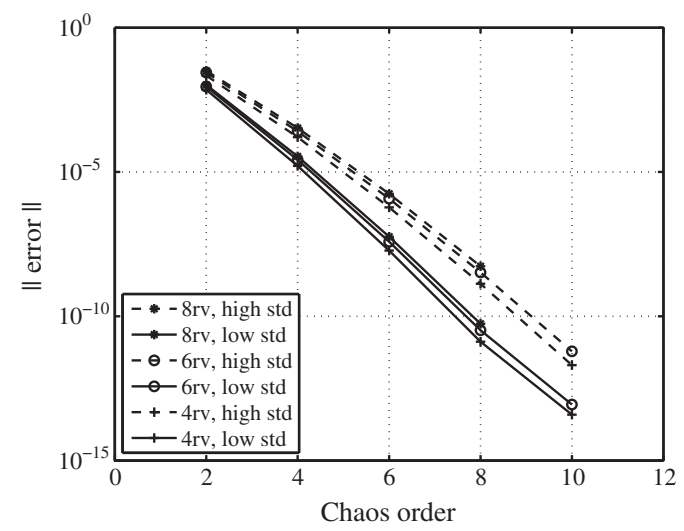

Figure 5. Convergence of the Frobenius error norm of the covariance matrix with respect to the chaos order, stochastic dimension, and input variability of the random field. The values of standard deviation are $\{0.10,0.20\}$ (low and high, respectively).

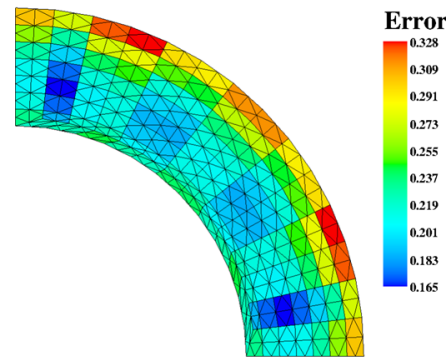

(a) $h_{\min }=6.6,4 \mathrm{rv}$

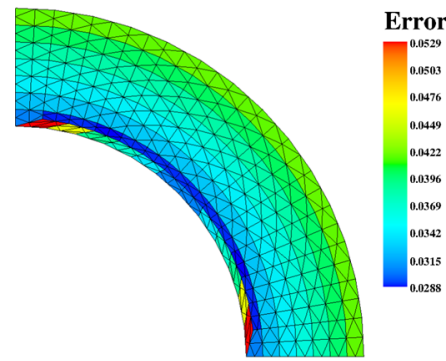

(d) $h_{\min }=6.6,200 \mathrm{rv}$

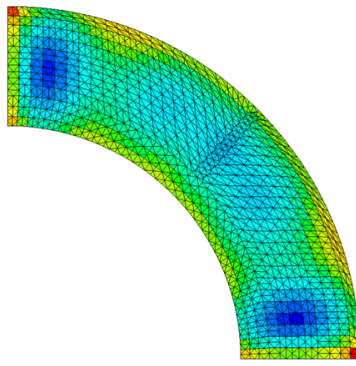

(b) $h_{\min }=2.9,4 \mathrm{rv}$

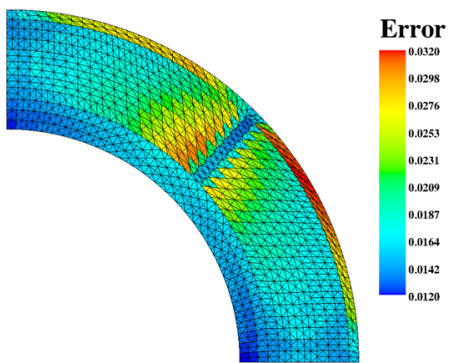

(e) $h_{\min }=2.9,200 \mathrm{rv}$
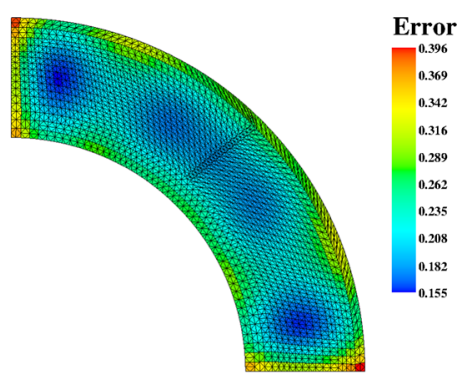

(c) $h_{\min }=1.9,4 \mathrm{rv}$

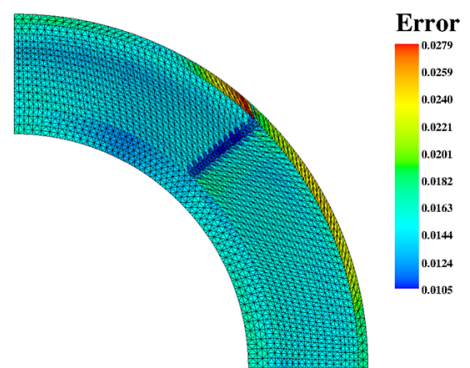

(f) $h_{\min }=1.9,200 \mathrm{rv}$

Figure 6. The spatial distribution of the $L^{2}$ error in approximating the input random field with two different stochastic space dimensions 4 and 200 ('rv' in the figure captions denotes random variables) for a fixed value of correlation length and three different mesh resolutions in ascending order $h_{\min }=6.6,2.9,1.9$. (a) $h_{\min }=6.6,4 \mathrm{rv}$; (b) $h_{\min }=2.9,4 \mathrm{rv}$; (c) $h_{\min }=1.9,4 \mathrm{rv}$; (d) $h_{\min }=6.6,200 \mathrm{rv}$; (e) $h_{\min }=2.9,200 \mathrm{rv;}$ and (f) $h_{\min }=1.9,200 \mathrm{rv}$.

Figure 7 shows the convergence trend of the error in the covariance kernel with decreasing mesh parameter $h_{\min }$ and increase in dimension of the stochastic space. The plotted error is a weighted average of the errors $\left(\varepsilon^{e}\right)$ shown as cell values in the color map in Figure 6, so that it can be written as $\|$ error $\|=\left(\sum_{i} A_{i}^{e} \varepsilon_{i}^{e}\right) /\left(\sum_{i} A_{i}^{e}\right)$. It shows that exponential convergence is achieved with $h_{\text {min }}$ and the slope is steepest for the highest input stochastic dimension. A similar behavior is observed for the study of convergence of error with increasing input stochastic dimension for a fixed mesh parameter $h_{\min }$, which shows that higher accuracy is obtained for fine mesh.

Figure 8 shows the contour plots of some realizations of the lognormal random diffusion coefficient obtained with a different number of terms retained in the discrete KL expansion. Here, we 


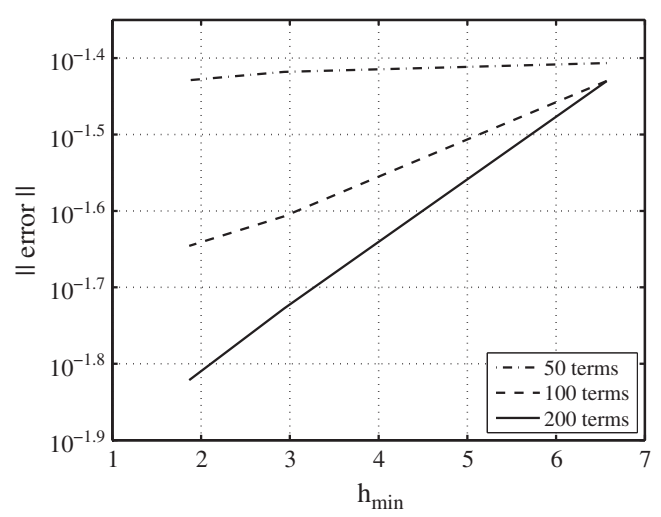

(a) Error convergence with mesh parameter size

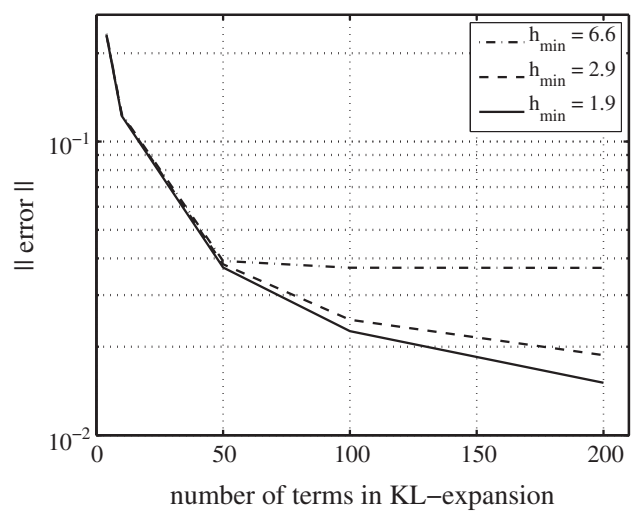

(b) Error convergence with the input stochastic space dimension

Figure 7. Error in the discretization of the covariance kernel for different mesh parameter size $\left(h_{\min }\right)$ and dimension of the input stochastic space. (a) Error convergence with mesh parameter size. (b) Error convergence with the input stochastic space dimension. KL, Karhunen-Loève.

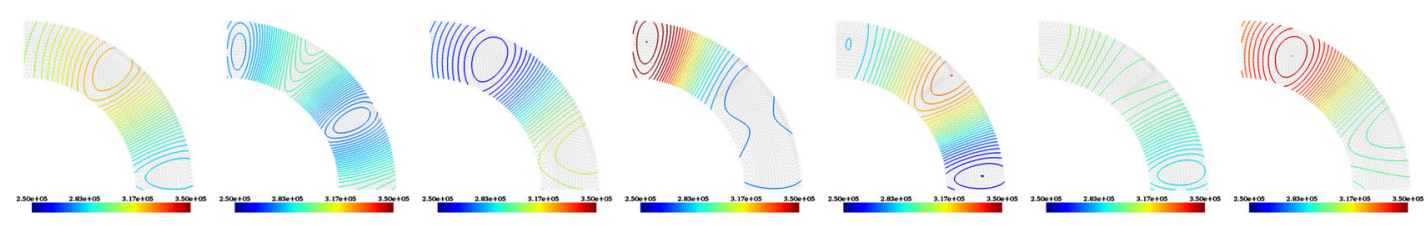

(a) Lognormal random field modeled with 4 random variables
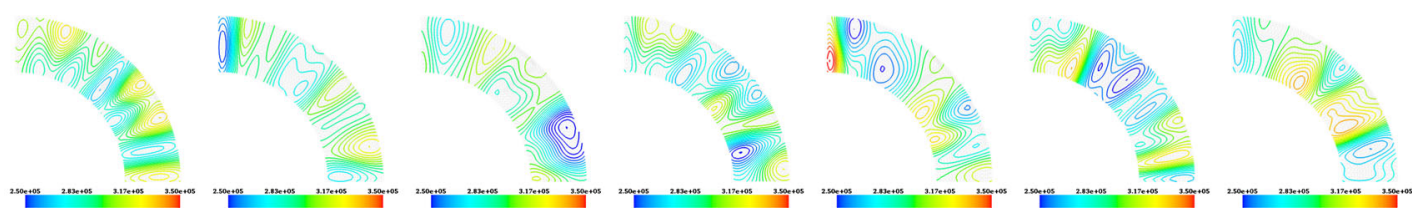

(b) Lognormal random field modeled with 20 random variables

Figure 8. The spatial distribution of the sample realizations of the lognormal stochastic diffusion coefficient modeled with (a) 4 and (b) 20 independent and identically distributed Gaussian random variables for exponential covariance kernel for a specific with different (decreasing) correlation lengths. The lognormal field is expressed with fourth-order Hermite polynomials. The colorbar limits in every subfigure in (a) and (b) is from $2.50 \mathrm{e}+05$ to $3.50 \mathrm{e}+05$.

have chosen decreasing correlation lengths for the random fields approximated with 4 and 20 random variables in Figure 8(a and b, respectively). The latter shows a greater degree of variation in the random-field realizations than the former.

Figure $9(\mathrm{a}-\mathrm{g})$ shows the eigenmodes of the spectral decomposition of an exponential covariance kernel $\left(C_{\text {exp }}\right)$, and Figure $9(\mathrm{~h}-\mathrm{n})$ the modes for a triangular covariance kernel $\left(C_{\text {tri }}\right)$ evaluated with the discrete KL expansion method, where

$$
C_{\exp }=\exp \left(-\left\|\mathbf{r}_{1}-\mathbf{r}_{2}\right\| / \mathbf{L}_{r}\right) \quad \text { and } \quad C_{\text {tri }}=1-\left\|\mathbf{r}_{1}-\mathbf{r}_{2}\right\| / \mathbf{L}_{r}
$$

The correlation length has been chosen to be $\mathbf{L}_{r}=\left[l_{r} / 4, l_{c} / 8\right]$, where the components of $\mathbf{L}_{r}$ give the correlation length along the radial and circumferential directions of the annular circular arc, respectively, and $\|\cdot\|$ denotes the $L^{2}$ norm.

While in the preceding example, the description of the random fields was provided with the $L^{2}$ norm of the distance between the points on the physical domain, the proposed method of obtaining the eigenfunctions at discrete points on the spatial domain (as presented in Section 3.1) can handle a variety of covariance descriptions of the random field on any arbitrary-shaped geometry. We present 


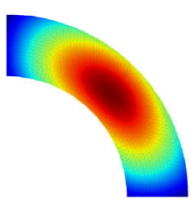

(a) Mode 01

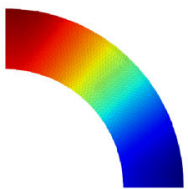

(h) Mode 01

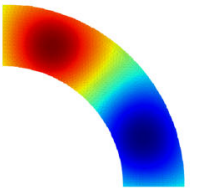

(b) Mode 02

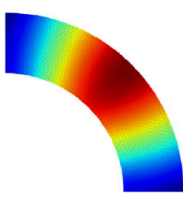

(i) Mode 02

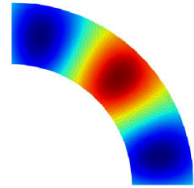

(c) Mode 03

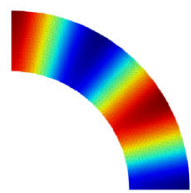

(j) Mode 03

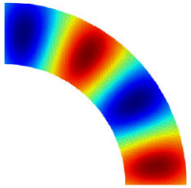

(d) Mode 04

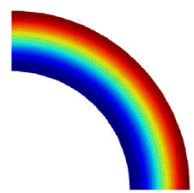

(k) Mode 04

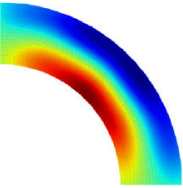

(e) Mode 08

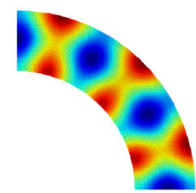

(1) Mode 08

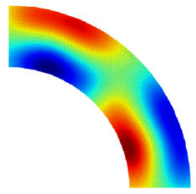

(f) Mode 10

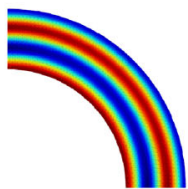

(m) Mode 10

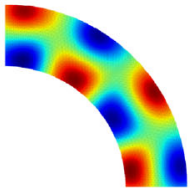

(g) Mode 14

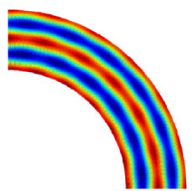

(n) Mode 14

Figure 9. The orthonormalized eigenmodes associated with the discrete Karhunen-Loève expansion of the exponential covariance kernel of a random field. Panels (a)-(g) are the eigenmodes for an exponential covariance kernel, (h)-(n) are the eigenmodes for a triangular covariance kernel. The correlation length has been taken as $\left[l_{r} / 4, l_{c} / 8\right]$ in both cases where $l_{r}$ and $l_{c}$ are the radial and circumferential lengths associated with the annular circular arc. (a) Mode 01. (b) Mode 02. (c) Mode 03. (d) Mode 04. (e) Mode 08. (f) Mode 10. (g) Mode 14. (h) Mode 01. (i) Mode 02. (g) Mode 03. (k) Mode 04. (l) Mode 08. (m) Mode 10. (n) Mode 14.

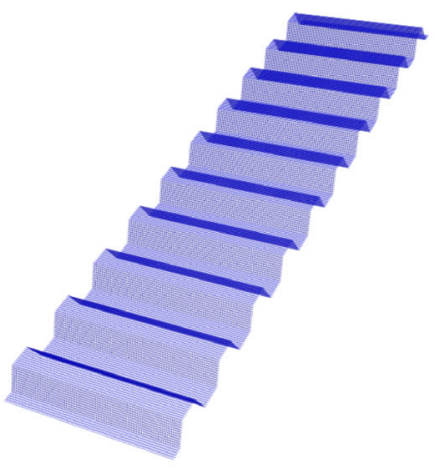

(a) A typical meshed corrugated panel with $C^{0}$ continuity of geometrical parameters across the corrugated edges.
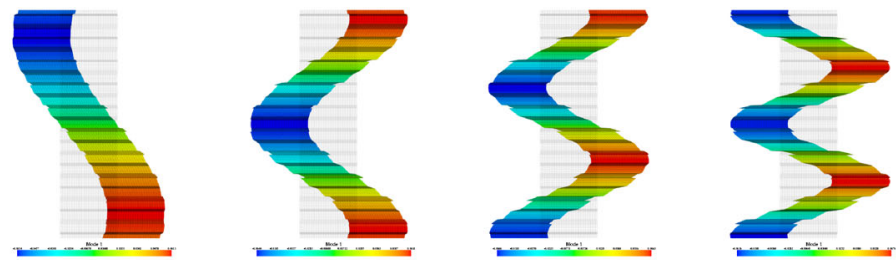

(b) Modes: $\{2,3,4,5\}$ for $L^{1}$ norm of the correlation length
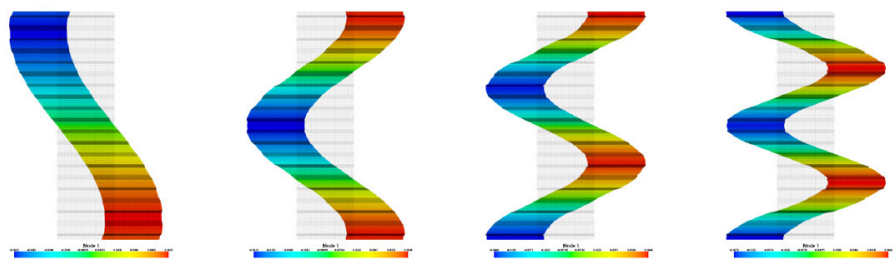

(c) Modes: $\{2,3,4,5\}$ for $L^{2}$ norm of the correlation length

Figure 10. The various normalized eigenmodes associated with the covariance function description of the random field over the spatial domain of a corrugated panel. The plots in (b) is for the case of the $L^{1}$ norm of the length used in the covariance function, while those in (c) is for the $L^{2}$ norm. The latter set of curves is smoother at the edges than the former set. (a) A typical meshed corrugated panel with $C^{0}$ continuity of geometrical parameters across the corrugated edges. (b) Modes $\{2,3,4,5\}$ for the $L^{1}$ norm of the correlation length. (c) Modes $\{2,3,4,5\}$ for the $L^{2}$ norm of the correlation length.

here a comparison of a random-field model on a corrugated panel that has been described with two different covariance functions. The $L^{1}$ norm is utilized to define the distance between two points of the panel along the corrugation (like the 'blue' curve in Figure 1(c)) in contrast to the $L^{2}$ norm or the Euclidean length (indicated by the 'red' curve). The models utilize these norms in the covariance function definition on the corrugated geometry as per Equation (64). Figure 10(a) shows a typical meshed corrugated panel over which a parameter is assumed to be randomly distributed. Figure 10(b and c) shows the top view of the plots of the random elastic stiffness parameter over the entire domain. The shaded gray area of the plot is the top view of the original undeformed corrugated panel on top of which a deformed color map of the panel has been superimposed. The deformed color map has been constructed such that the various eigenfunctions associated with the discrete 
KL expansion are plotted as sideways displacements of the nodal values of the eigenfunctions of the covariance function. The plots in Figure 10(b) correspond to the $L^{1}$ norm of lengths used in the covariance function, while those in Figure 10(c) are for the $L^{2}$ norm of length. For the covariance function defined with the $L^{2}$ norm, the eigenfunctions are smoother at the edges of the corrugation (which is $C^{0}$ continuous) compared with the eigenfunctions obtained for the case of $L^{1}$ norm.

Figure 11 gives a comparison of the eigenvalues of the spectral decomposition of the covariance function of the input random field over the spatial domain for two different mesh resolutions and two different correlation lengths, $L / 4$ and $L / 20$. Here, $L$ denotes the characteristic length for this spatial domain given by the square root of the area of the domain. The eigenvalue spectrum shows that the eigenvalue approximations are in good agreement at least up to the first few hundred indices and the approximation is improved as the mesh resolution is improved. Also, for small correlation lengths and coarse meshes (as seen in Figure 11), the deviation is more pronounced. This suggests that for a low-order approximation of the random field with the KL eigenfunctions, the 'discrete KL expansion' performs quite well.

Figure 12 shows the error in resolving the Fredholm integral equation using the methods of KL expansion at discrete points in space and the FE-type solution techniques. The spectral decomposition has been performed with two different mesh resolutions, which correspond to the curves shown in Figure 11. The relative error has been estimated with respect to an exact covariance matrix estimated at the Gauss quadrature integration points within each element. The results have been compared with the approximate covariance matrices synthesized from the following: (i) the random-field representation obtained using the discrete-type KL and (ii) the Galerkin FE-type method. This is as per Equation (62). Here, we consider two different mesh resolutions, a coarse mesh and a refined one, and two different correlation lengths. The results indicate that good levels of approximation is obtained with both methods and the level of accuracy is comparable.

\subsection{System response}

We present here numerical examples to demonstrate the applicability of the methods proposed in Sections 4 and 5 for handling parametric uncertainty with discrete representation of the random field and for tackling the diffusion equation on a domain with random boundary fluctuations. The results for the steady-state diffusion equation with parametric uncertainty is presented in Section 7.2.1. The case of unsteady diffusion on a domain with a random boundary has been dealt with in Section 7.2.2.

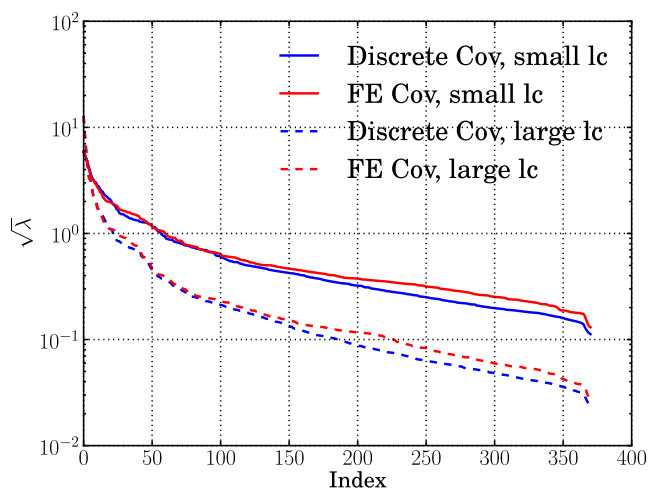

(a) Course mesh

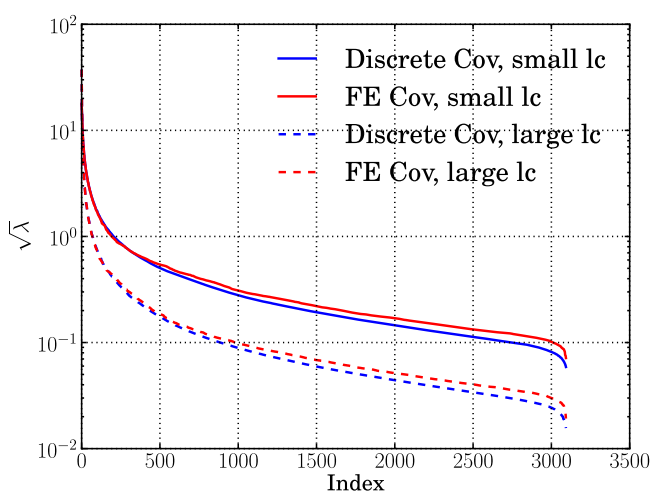

(b) Fine mesh

Figure 11. Comparison of the descending eigenvalue spectrum associated with the exponential covariance kernel with two different mesh resolutions of the random parametric field obtained with the discrete KL expansion method (marked as 'Discrete Cov') and the finite element (FE)-type solution technique (marked as 'FE Cov'). Two different correlation lengths $L / 4$ and $L / 20$ have been considered here, which correspond to the 'large lc' and 'small lc', respectively. The input standard deviation is $\sigma_{a}=0.5$. (a) Course mesh. (b) Fine mesh. 

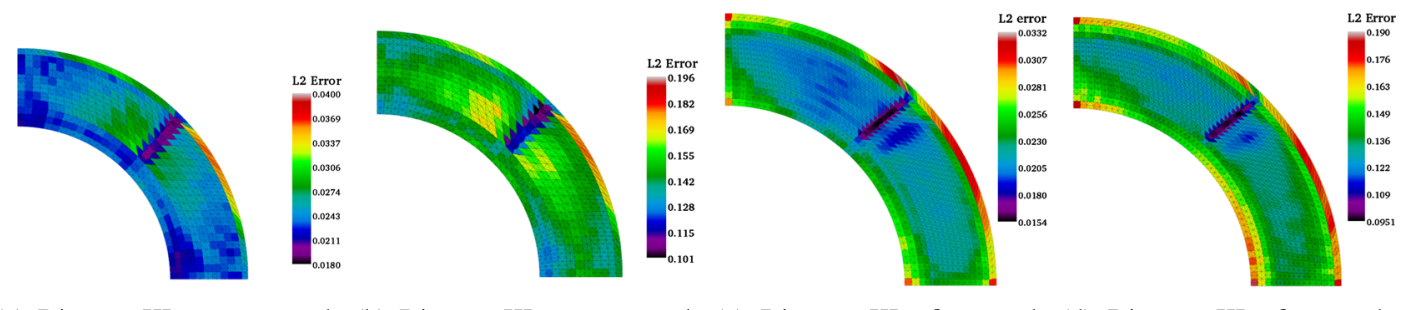

(a) $\mathrm{Di}$
$\mathrm{cl}=L / 4$

(b) Discrete

(c) Discr

(d) Discrete KL, fine mesh,

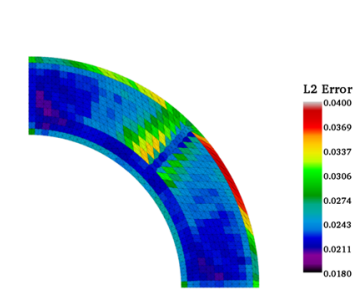

$$
\text { la } / 20
$$$$
\mathrm{cl}=L / 4
$$

$\mathrm{cl}=L / 20$

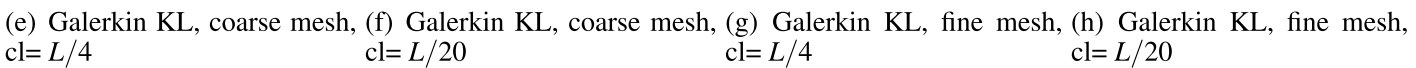

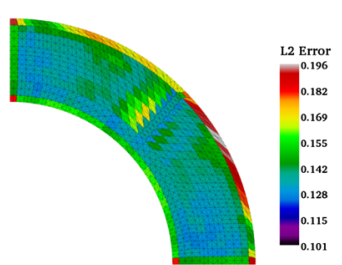

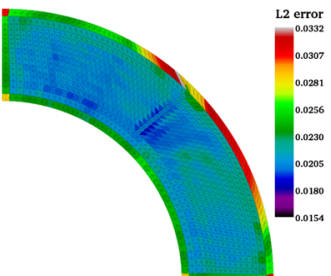

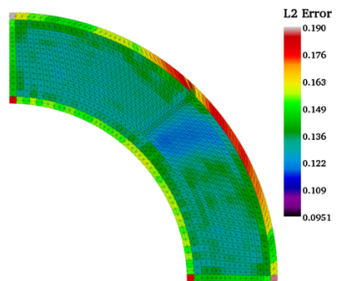

Figure 12. Comparison of the $L^{2}$ error of the covariance matrices constructed with the random field obtained from the Karhunen-Loève (KL) eigenfunctions. The KL eigenfunctions have been resolved with the proposed 'discrete' spectral decomposition and the finite element-type 'Galerkin' method. Two different mesh resolutions have been considered here as indicated by the 'coarse' and 'fine' meshes. 'cl' denotes the correlation length of the input parametric random field, and two distinct values have been used, $L / 4$ and $L / 20$, where $L$ is the characteristic length of the domain. (a) Discrete KL, coarse mesh, $\mathrm{cl}=L / 4$. (b) Discrete $\mathrm{KL}$, coarse mesh, $\mathrm{cl}=L / 20$. (c) Discrete KL, fine mesh, $\mathrm{cl}=L / 4$. (d) Discrete KL, fine mesh, $\mathrm{cl}=L / 20$. (e) Galerkin KL, coarse mesh, cl $=L / 4$. (f) Galerkin KL, coarse mesh, cl $=L / 20$. (g) Galerkin KL, fine mesh, $\mathrm{cl}=L / 4$. (h) Galerkin KL, fine mesh, $\mathrm{cl}=L / 20$.

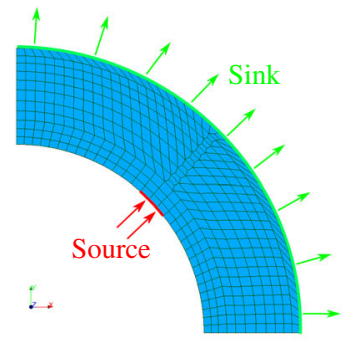

(a) Configuration of the steady state physical system
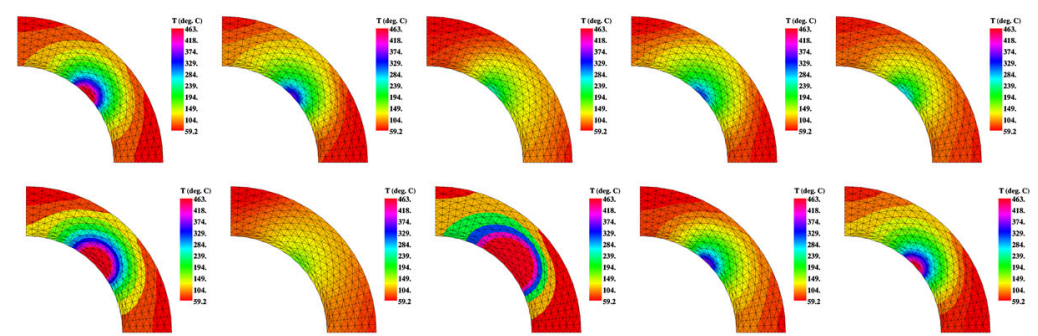

(b) Sample realizations of the steady state response of the randomly parametrized system

Figure 13. (a) The configuration of the steady-state physical system with the boundary conditions implemented at the displayed locations. (b) The various sample realizations of the steady-state response for a lognormal input random parameter approximated with 20 random variables. The colorbar limits in every subfigure is 59.2 to 463 .

7.2.1. Steady-state system response. We present here the results obtained for a steady-state diffusion problem with random lognormal diffusion coefficient. Here, we have not considered any perturbations of the random geometry. The covariance function of the random diffusion coefficient is assumed to be exponential with the correlation length, 'cl', defined as the $L^{2}$ norm of the difference in position coordinates of $\mathrm{FE}$ nodes, where $\mathrm{cl}=L / 20$.

Figure 13(a) is a schematic diagram of the steady-state physical system with the source and sink terms denoting the input flux to the system and the dissipation from the system, respectively. Figure 13(b) shows the various sample realizations of the steady-state system response with random diffusion coefficient. The configuration of the deterministic system is such that the response is symmetrical in the angular direction. 
Figure 14 shows the statistics of the response over the spatial domain. Figure 14(a) shows the contour plot of the deterministic response field and compares it with the mean and standard deviation of the response given in Figure 14(b and 14c), respectively. The results show that the mean response is symmetric in the angular direction. Also for the lognormal input random field, the mean response shows that more energy is concentrated near the source flux. The plot for the standard deviation also shows the highest variability of the response near the source.

7.2.2. Unsteady-state response on random domain. In this section, we take the example of an unsteady diffusion system on a physical domain with random topology perturbations to demonstrate the computational methodology proposed in Section 5. Following from Equation (25), we write the unsteady diffusion equation on the random domain as

$$
\begin{aligned}
C \dot{T}(t)+\nabla(K \nabla T(t)) & =Q(t) \quad \text { on } \mathscr{D}_{\theta} \quad \forall t \in[0, T] \\
u=G_{c} \quad \text { on } \Gamma_{\theta}^{0} ; \quad \mathbf{n}_{1} \cdot \nabla T(t) & =q_{1}(t) \quad \text { on } \Gamma_{\theta}^{1} \quad \text { and } \quad \mathbf{n}_{2} \cdot \nabla T(t)=q_{2}(t) \quad \text { on } \Gamma_{\theta}^{2} \quad \forall t \in[0, T] \\
T(t=0) & =T_{0} \quad \text { on } \mathscr{D}_{\theta}
\end{aligned}
$$

where $\Gamma_{\theta}^{1}$ is the 'red' part of the boundary in Figure 16(a), which supplies the input flux to the system, and $\Gamma_{\theta}^{2}$ denotes the 'green' portion of the boundary, which dissipates energy. $G_{c}$ is the prescribed value of the Dirichlet boundary condition, and $q_{1}$ and $q_{2}$ are the boundary flux terms. $T_{0}$ is the initial condition. All the boundary and initial conditions have been taken to hold true for every random sample. The preceding equation indicates that the initial and boundary conditions are prescribed on the boundary for every sample realization of the random boundary. The time integration is carried out using Euler's central difference scheme, which is an implicit time-stepping algorithm with a fixed time step size. The upper bound on the step size is governed by the dynamic characteristics of the transient system and has been chosen to be sufficiently small to ensure stability and convergence.

Figure 15 shows two sample realizations of domains with random boundary. The boundary fluctuation has been modeled as a Gaussian random field and described with an exponential covariance function between the coordinates of the nodes of the element lying on the boundary. The correlation length has been chosen as $L / 4$, where $L$ is the characteristic length of the domain. The discretized model of the random field has been represented with 20 random variables. The boundary perturbations of the two samples are geometrically distinct from each other, as highlighted in Figure 15(c).

The plot in Figure 16(a) shows the configuration of the transient dynamic system on a random domain (the sample realizations of which have been shown in Figure 15) with the 'red' arrows indicating the region of input energy while the 'green' arrows indicate the boundary from which energy is dissipated. The profile of the input energy can be written as $q_{1}=Q_{0} \exp \left(-c_{t} t\right)$, where $Q_{0}$ is the flux at $t=0$ and $c_{t}>0$ is a constant signifying the exponential rate of decay of the input flux with time $t$. Here, $c_{t}$ has been chosen to be 0.3 . Also, the output flux is given by $q_{2}(t)=C_{h}\left(T-T_{a}\right)$,

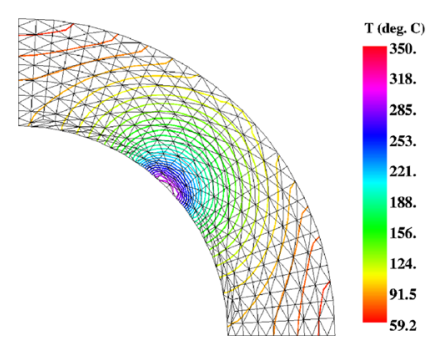

(a) Deterministic steady state response

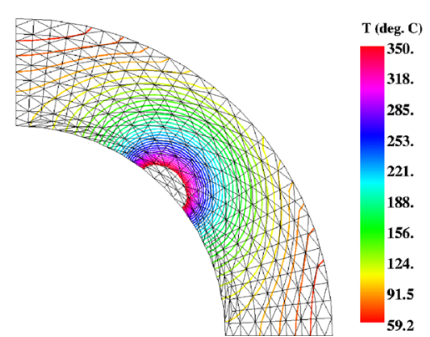

(b) Mean Steady State response

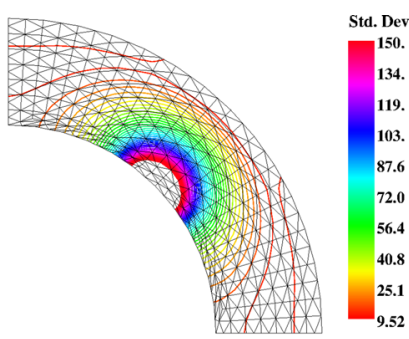

(c) Standard deviation of the steady state response

Figure 14. The spatial distribution of the steady-state response field. (a) Deterministic steady-state response. (b) Mean steady-state response. (c) Standard deviation of the steady-state response. 


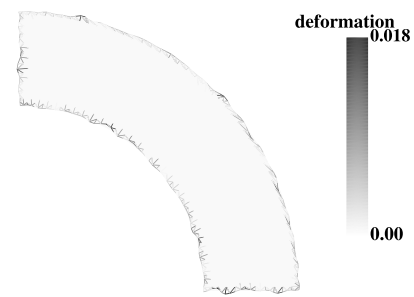

(a) Sample 1

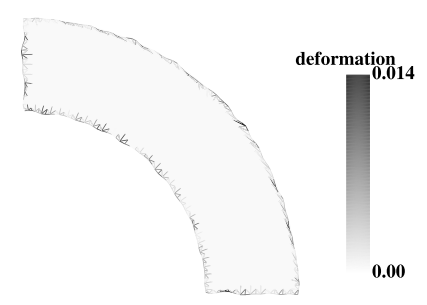

(b) Sample 2

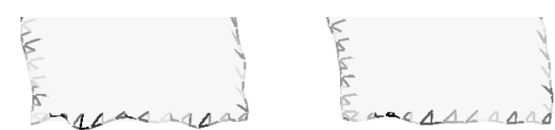

(c) Horizontal boundary of Sample 1 and 2
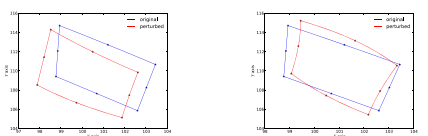

(d) Element perturbations

Figure 15. (a-b) Sample realizations of the domain with random boundary. The boundary fluctuations have been modeled as a Gaussian random field with an exponential covariance, which incorporates the correlation of the position coordinates of the nodes of the element lying on the boundary. The input standard deviation is $\sigma_{a}=0.5$. (c) A magnified view of the sample realizations of the bottom horizontal boundary shown in (a-b). (d) Sample realizations of the perturbations of a particular element lying on the random horizontal boundaries shown in (c). The 'blue' element constitutes the baseline model, while the 'red' ones are the perturbed configurations corresponding to the two samples shown.

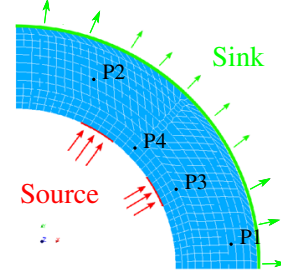

(a) Configuration of the transient system

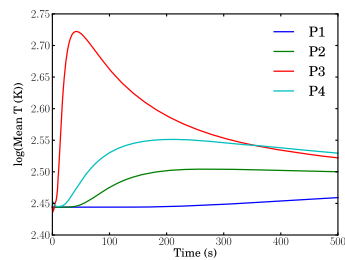

(b) Mean Response

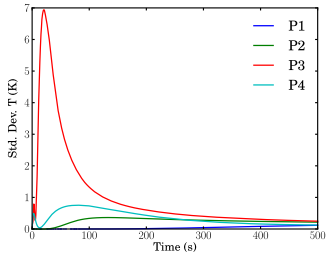

(c) Standard Deviation

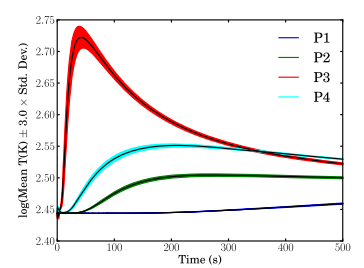

(d) Mean $\pm 3 \times$ Std. Dev.

Figure 16. (a) Configuration of the unsteady diffusion system. (b, c) Time history of the mean and standard deviation $\sigma$ of the response at arbitrarily chosen points in the domain. (d) Mean with $\pm 3 \times \sigma_{a}$ envelope around it. The input standard deviation is $\sigma_{a}=0.5$.

where $C_{h}$ is a positive constant with $T$ being the response field and $T_{a}=278$ being a fixed constant. Thus, the rate of dissipation of energy is given by the difference $T-T_{a}$. The initial condition has been chosen as $T(t=0)=278$. The time integration has been carried out over the interval $t=[0,500] \mathrm{s}$. The mean and standard deviation of the response have been plotted in Figure 16(b and c), which gives the time histories of the preceding response statistics at arbitrarily chosen points in the domain. Figure 16(d) gives the mean plotted with a $\pm 3 \times \sigma$ envelope around it. Given the initial and boundary conditions, it is expected that the response would first increase with the input flux and then gradually decay and tend toward a uniform value over the entire domain.

Figure 17 gives the snapshots of the statistics of unsteady response plotted in the mean random domain at time intervals shown in the figure. Figure 17(a) shows that the response first grows with time at the positions of input flux and slowly decays with the time as the input flux decreases. The standard deviation plots in Figure 17(b) when compared with the plot in Figure 16(c) show that evolution of the standard deviation of the response field follows the mean response pattern closely. However, it can be observed that as the response decays, the highest standard deviation is concentrated in the regions of the input flux along the radial direction of the circular arc. This is expected because the randomness in the boundary edges propagated to the response and evolving in time would be concentrated along the dominant directions of the spatial gradient of the solution (which is the radial direction in this case).

The convergence of the linear system to be solved at each time step is studied here with regard to the number of iterations required for the solution to converge to the desired accuracy. The CG algorithm, which is a Krylov-type iterative solver, has been used here in conjunction with blockdiagonal preconditioners for the linear system solution at each time step. The previous time step 

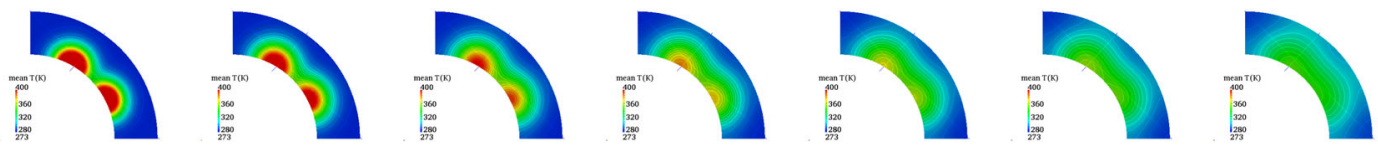

(a) Mean response at $t=\{125,175,225,275,325,375,450\}$ seconds
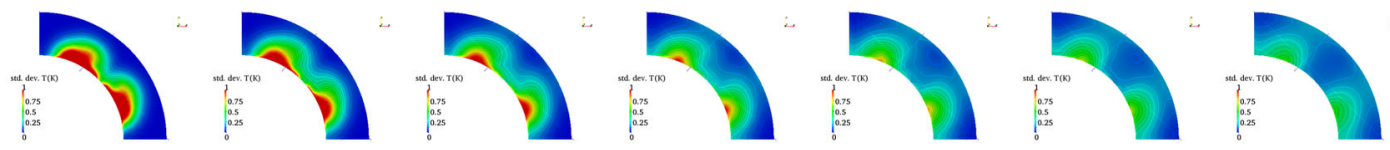

(b) Standard deviation of the response at $t=\{125,175,225,275,325,375,450\}$ seconds

Figure 17. Transient response of the mean and standard deviation $\left(\sigma_{T}\right)$ of the stochastic field on a domain with random boundary under the action of external input flux. The boundary fluctuations have been modeled as a Gaussian random field with an exponential covariance that incorporates the correlation of the position coordinates of the nodes of the element lying on the boundary. Twenty independent and identically distributed Gaussian random variables have been used to model the random boundary. The input standard deviation is $\sigma_{a}=0.5$. (a) Mean response at $t=\{125,175,225,275,325,375,450\}$ s. (b) Standard deviation of the response at $t=\{125,175,225,275,325,375,450\}$ s. The colorbar limits in all subfigures in (a) is 273 to 400 while that in (b) is 0 to 1 .

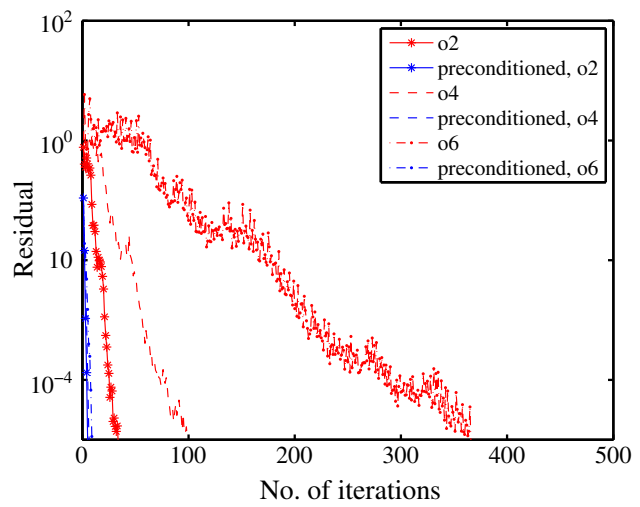

(a) Residual for CG iterations for input std. dev. 0.25

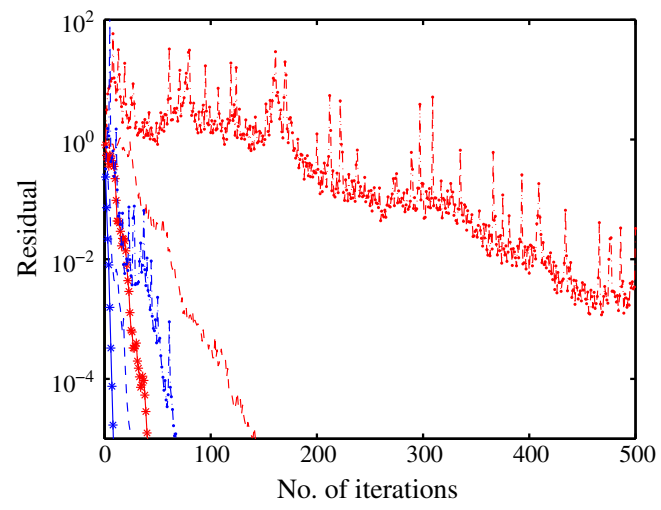

(b) Residual for CG iterations for input std. dev. 0.50

Figure 18. Convergence of the solution using an iterative CG scheme with and without the use of preconditioners (block diagonal) for different orders of expansion of the solution in the stochastic Hilbert space and for two different values of input standard deviation of the random field, $\sigma_{a}=\{0.25,0.50\}$. (a) Residual for $\mathrm{CG}$ iterations for an input standard deviation of 0.25. (b) Residual for CG iterations for an input standard deviation of 0.50 .

solution is used as a starting guess for the solution at the current time step. Here, we present the calculations that have been performed with different orders of expansion of the solution with the orthogonal Hermite polynomials and different values of input standard deviation of the random field.

Figure 18 shows the plots of the residuals given in Table II for two different values of input standard deviation of the random field. It shows that more iterations are necessary when the order chaos expansion of the solution is increased and also for an increase in the input variability of the random field. It also shows the efficacy of the preconditioning technique, which enables rapid convergence to the solution even for cases of high standard deviation and input variability where the cost for the case of 'without preconditioner' becomes prohibitively high.

Table III shows a comparison of the $L^{2}$ relative error of the mean and standard deviation of the response on the domain with random boundary obtained with various orders of chaos expansion of the solution. The direct Monte Carlo simulation with 10,000 samples has been taken as the benchmark solution here with respect to which $L^{2}$ relative error has been constructed. The comparison of the various levels of residual of the linear system with the $L^{2}$ relative error highlights the important aspect of convergence of the solution with the order of chaos expansion. It can be seen from the table 
Table II. Convergence behavior of the CG method with and without block-diagonal preconditioners for solving the linear system obtained from the block-sparse coefficient matrix for the diffusion operator on a domain with random boundary.

\begin{tabular}{|c|c|c|c|c|c|c|c|c|}
\hline \multirow{3}{*}{$\begin{array}{l}\text { PC } \\
\text { order }\end{array}$} & \multicolumn{4}{|c|}{ Without preconditioner } & \multicolumn{4}{|c|}{ With preconditioner } \\
\hline & \multicolumn{2}{|c|}{$\mathrm{SD}=0.25$} & \multicolumn{2}{|c|}{$\mathrm{SD}=0.50$} & \multicolumn{2}{|c|}{$\mathrm{SD}=0.25$} & \multicolumn{2}{|c|}{$\mathrm{SD}=0.50$} \\
\hline & Residual & Iterations & Residual & Iterations & Residual & Iterations & Residual & Iterations \\
\hline 2 & $7.91 \times 10^{-6}$ & 34 & $8.85 \times 10^{-6}$ & 41 & $9.73 \times 10^{-6}$ & 5 & $4.45 \times 10^{-6}$ & 9 \\
\hline 4 & $9.62 \times 10^{-6}$ & 99 & $6.62 \times 10^{-6}$ & 144 & $4.76 \times 10^{-6}$ & 7 & $5.42 \times 10^{-6}$ & 25 \\
\hline 6 & $8.99 \times 10^{-6}$ & 366 & $3.26 \times 10^{-2}$ & 500 & $6.95 \times 10^{-6}$ & 10 & $7.19 \times 10^{-6}$ & 68 \\
\hline 8 & $5.87 \times 10^{-4}$ & 500 & $7.47 \times 10^{-1}$ & 500 & $6.77 \times 10^{-6}$ & 14 & $7.12 \times 10^{-6}$ & 184 \\
\hline
\end{tabular}

SD, standard deviation.

Table III. Convergence of the relative $L^{2}$ error of the mean and variance of the response with respect to the direct Monte Carlo simulation results and its relation with the residual error of the linear system for various orders of chaos expansion of the response of the diffusion system on a domain with random boundary.

\begin{tabular}{lccccccc}
\hline \multirow{2}{*}{$\begin{array}{l}\text { PC } \\
\text { order }\end{array}$} & \multicolumn{2}{c}{ Input standard deviation $=0.25$} & & \multicolumn{3}{c}{ Input standard deviation $=0.50$} \\
\cline { 2 - 3 } \cline { 7 - 8 } & Residual & Error (mean) & Error (variance) & & Residual & Error (mean) & Error (variance) \\
\hline 2 & $7.91 \times 10^{-6}$ & $9.87 \times 10^{-5}$ & $3.51 \times 10^{-3}$ & & $8.85 \times 10^{-6}$ & $4.99 \times 10^{-4}$ & $1.64 \times 10^{-2}$ \\
4 & $8.62 \times 10^{-6}$ & $2.53 \times 10^{-5}$ & $3.30 \times 10^{-3}$ & & $6.62 \times 10^{-6}$ & $1.33 \times 10^{-4}$ & $1.03 \times 10^{-2}$ \\
6 & $9.39 \times 10^{-6}$ & $2.56 \times 10^{-5}$ & $3.30 \times 10^{-3}$ & & $4.62 \times 10^{-4}$ & $9.4 \times 10^{-5}$ & $3.4 \times 10^{-3}$ \\
8 & $5.13 \times 10^{-6}$ & $2.55 \times 10^{-5}$ & $3.30 \times 10^{-3}$ & & $3.09 \times 10^{-2}$ & $6.96 \times 10^{-5}$ & $4.0 \times 10^{-3}$ \\
10 & $9.77 \times 10^{-6}$ & $2.57 \times 10^{-5}$ & $3.30 \times 10^{-3}$ & & 1.19 & $7.39 \times 10^{-4}$ & $2.54 \times 10^{-2}$ \\
\hline
\end{tabular}

that for an input standard deviation of $\sigma_{a}=0.25$ and for residuals of the order of $\approx 10^{-6}$, the relative error gives a satisfactory level of convergence of the mean and standard deviation of the solution from the fourth-order chaos. However, for the case of $\sigma_{a}=0.50$, it is seen that for low-order chaos and for residuals of $\approx 10^{-6}$ (which is rapidly reached because the dimension of the linear system is quite small for low-order chaos), the levels of relative error are larger than its higher-order counterparts. For the higher-order chaos, it is seen that relatively high levels of residuals produces accuracy levels of $\approx 10^{-4}-10^{-5}$ and $\approx 10^{-2}-10^{-3}$ for the mean and standard deviation, respectively. Thus, even a poorly converged linear solver for high-order chaos expansion can produce first-moment and second-moment accuracy levels comparable with that produced by a converged linear solver for low-order chaos.

\subsection{Implementational requirements and limitations}

While the proposed discrete KL expansion for the approximation of random fields on arbitraryshaped domains is seen to perform well, it has to be mentioned that the approximation accuracy of the random field is a function of the mesh resolution. When using a higher number of KL modes in the approximate random-field model, it is essential to increase the mesh resolution to capture the complex deformation shapes. Additionally, the extension of the discrete KL expansion to cases where the field cannot be expressed in terms of its second-moment properties is necessary. Finite-dimensional representation of a general non-Gaussian random field is in general a non-trivial exercise. The proposed discrete KL expansion can be extended to work within the framework of the analytico-simulative techniques used to match the target distribution functions for such cases.

The stochastic isoparametric mapping proposed here depends on the random modeling of the nodal coordinates, which in turn is described by their correlation structure. Hence, the formulation does not depend explicitly on a set of geometrical design variables that have been randomly parameterized to obtain the random topology perturbations. The method can be applied to non-simply connected domains (such as a surface with holes, as shown in Figure 3). However, the method cannot 
be used to analyze the cases where discontinuities (such as cracks, holes, and notches) develop in random numbers and locations in the physical domain, which has not already been modeled in the deterministic baseline model. Additionally, the proposed method works under the assumption that the perturbations in the topology are captured satisfactorily by parameterizing the mesh adjacent to the random boundary. The baseline model should not be distorted to the extent that a remeshing becomes unavoidable. While this assumption holds in many practical scenarios, the method would fail to perform satisfactorily and would be computationally expensive when large fluctuations of the domain boundary are considered. Additionally, the stochastic spectral Galerkin method used here gives accurate prediction of the second-moment characteristics of the response but requires high-order chaos expansions to approximate the tail probabilities, which are useful for rare-event predictions. This should be taken into account while performing the stochastic analysis using stochastic Galerkin methods with finite-order chaos expansion.

\section{CONCLUSION AND FUTURE WORK}

The numerical method proposed here is a unified approach to solve the randomly parameterized PDEs on spatial domains with random topology perturbations. The finite-dimensional random-field representation has been obtained with the discrete KL expansion. This description has been utilized to develop a unified framework for the resolution of the response statistics of these random systems within the stochastic spectral Galerkin framework. The salient features of the present work can be summarized as follows:

- A discrete spectral decomposition of the covariance matrix to obtain the KL expansion of the random field has been proposed here, which evaluates the random field at the discrete FE nodes of an unstructured mesh. The accuracy of this expansion has been validated with a relative error metric.

- The explicit expressions for the coefficients of the lognormal random field have been derived in Appendix A. This enables rapid evaluation of the coefficients of the lognormal field with Hermite polynomials of i.i.d. Gaussian random variables.

- PDEs on domains with random boundary have been studied within the framework of the discrete spectral decomposition of the random boundary perturbation field with a proposed stochastic isoparametric mapping.

- The mapping of the differential operators and the associated Jacobian matrices has been expressed explicitly with random polynomials in order to facilitate prior computation of the expectations of stochastic polynomials for use with the stochastic Galerkin method.

- It has been shown that the computational complexity associated with the evaluation of the integrals in the weak formulation using the full stochastic tensor quadrature grows exponentially with the dimension and order of chaos expansion of the random field. This highlights the necessity of precomputing the tensor inner products even for moderate orders of chaos expansion.

- The proposed stochastic mapping method has been used in conjunction with the implicit central difference time-stepping algorithm to obtain the second-order statistics of an unsteady diffusion system on a domain with random boundary.

- The accuracy of the response statistics has been studied with respect to brute-force Monte Carlo simulations (which serves as the benchmark solution) for various orders of chaos expansions, numbers of quadrature integration points, and the corresponding computational cost.

Thus, a discrete spectral resolution of the covariance matrix gives an accurate representation of the random field, which allows us to compute the KL eigenmodes for all practical descriptions of the covariance functions of the random field on all geometrical domains. For the case of domains with random topology, by using the deformation of the meshed region adjacent to the boundary surface, it is possible to capture the boundary deformation without remeshing each random realization of the domain. For low-order chaos expansion and small input stochastic space dimensions, quadrature schemes may be implemented with the stochastic Galerkin scheme. However, for 
moderate/high-dimensional stochastic problems with comparable order of chaos expansion, precomputing the statistics of the stochastic polynomials is the computationally feasible approach. This has been discussed in detail in Section 5 .

Future work would look to extend the proposed stochastic isoparametric mapping to non-linear problems on random domains where arbitrarily small perturbation of the domain boundary may result in a significant modification of the response field. Also, comparison of the computational efficacy of high-dimensional stochastic problems where the stochastic integration is performed with dimension-adaptive sparse-grid algorithms would be another important aspect of future study. Lastly, the applicability of various emulation methods, such as the Gaussian process emulator, can be investigated for their potential application in the study of SPDE on random domains.

\section{APPENDIX A: DERIVATION OF CLOSED-FORM EXPRESSIONS FOR THE FINITE-ORDER CHAOS REPRESENTATION OF LOGNORMAL RANDOM FIELDS}

The derivation of the analytical expressions for the undetermined coefficients associated with the stochastic polynomial expansion of the lognormal field is presented here. We assume a lognormal random field $\mathbf{a}_{l}(\theta)=\exp \left(\overline{\mathbf{a}}_{0}+\sum_{i=1}^{M} \xi_{i}(\theta) \tilde{\mathbf{a}}_{i}\right)$ in a discretized spatial domain such that $\mathbf{a}_{l}(\theta) \in$ $\mathbb{R} n$. For the sake of computational convenience, we express this random field as a series expansion of finite-order multivariate Hermite polynomials $\mathscr{H}_{i}(\xi(\theta))$ spanning the $M$-dimensional stochastic hyperspace $\Theta^{(M)}$ as

$$
\mathbf{a}_{l}=\sum_{i=0}^{m} \mathbf{a}_{i}^{l} \mathscr{H}_{i}(\xi(\theta)) \quad \text { where } \quad \mathbf{a}_{i}^{l}=\frac{\left\langle\mathbf{a}_{l}(\theta) \mathscr{H}_{i}\left(\xi(\theta)_{L^{2}\left(\Theta^{(M)}, d P_{\xi}\right)}\right\rangle\right.}{\left\langle\mathscr{H}_{i}\left(\xi(\theta)_{L^{2}\left(\Theta^{(M)}, d P_{\xi}\right)}^{2}\right\rangle\right.}
$$

The aim is to express the undetermined coefficients as a closed-form analytical expression in terms of the Gaussian i.i.d. random variables $\xi=\left\{\xi_{1}, \ldots, \xi_{M}\right\}$.

\section{Theorem 1}

If a lognormal random field $\mathbf{a}_{l}(\theta)$ is expanded as a series of multivariate orthogonal Hermite polynomials spanning the stochastic Hilbert space of the input Gaussian random variables, then the undetermined coefficients associated with the individual polynomial terms are given by

$$
\mathbf{a}_{i}^{l}=\exp \left(\overline{\mathbf{a}}_{0}\right) \prod_{i=1}^{M} \exp \left(\frac{1}{2} \tilde{\mathbf{a}}_{i}^{2}\right) \frac{\mathscr{H}_{i}^{h d}\left(\tilde{\mathbf{a}}_{1}, \ldots, \tilde{\mathbf{a}}_{M}\right)}{\left\langle\mathscr{H}_{i}(\xi(\theta)\rangle_{L^{2}(\Theta(M)}^{2}, d P_{\xi}\right)}
$$

where $\mathscr{H}_{i}^{\text {hd }}\left(\tilde{\mathbf{a}}_{1}, \ldots, \tilde{\mathbf{a}}_{M}\right)$ is the vector of the highest-order term associated with the $i$ th Hermite polynomial and $\left(\tilde{\mathbf{a}}_{1}, \ldots, \tilde{\mathbf{a}}_{M}\right)$ is the $M$ discrete eigenvectors of the spectral decomposition of the covariance kernel of the input random field.

Proof

We begin by noting that the $p$ th-order multivariate Hermite polynomials are obtained using the generating function

$$
\begin{aligned}
\mathscr{H}_{i}\left(\xi_{1}^{p_{1}}, \ldots, \xi_{M}^{p_{M}}\right) & =(-1)^{p_{1}+\ldots+p_{M}} \exp \left(\frac{1}{2} \sum_{j=1}^{M} \xi_{i}^{2}\right) \frac{\partial^{p_{1}}}{\partial \xi^{p_{1}}} \cdots \frac{\partial^{p_{M}}}{\partial \xi^{p_{M}}} \exp \left(-\frac{1}{2} \sum_{j=1}^{M} \xi_{i}^{2}\right) \\
\text { where } p & =p_{1}+\cdots+p_{M}
\end{aligned}
$$


where $p_{1}, \ldots, p_{M}$ are the degree of the random variables $\xi_{1}, \ldots, \xi_{M}$, respectively. Hence, the expression for $\mathbf{a}_{i}^{l}$ from Equation (A.1) can be written after some simplification as the integration in stochastic space as

$$
\begin{aligned}
& \mathbf{a}_{i}^{l}=\frac{(-1)^{p}(2 \pi)^{-\frac{M}{2}}}{\left\langle\mathscr{H}_{i}(\xi(\theta)\rangle_{L^{2}\left(\Theta^{(M)}, d P_{\xi}\right)}^{2}\right.} \int_{-\infty}^{+\infty} \cdots \int_{-\infty}^{+\infty} \exp \left(\overline{\mathbf{a}}_{0}+\sum_{i=1}^{M} \xi_{i} \tilde{\mathbf{a}}_{i}\right) \frac{\partial^{p_{1}}}{\partial \xi_{1}^{p_{1}}} \\
& \cdots \frac{\partial^{p_{M}}}{\partial \xi_{M}^{p_{M}}}\left\{\exp \left(-\frac{1}{2} \sum_{j=1}^{M} \xi_{i}^{2}\right)\right\} d \xi_{1} \cdots d \xi_{M} \\
& =\mathscr{C} \int_{-\infty}^{+\infty} \cdots \int_{-\infty}^{+\infty} \prod_{i=1}^{M} \exp \left(\xi_{i} \tilde{\mathbf{a}}_{i}\right) \frac{\partial^{p_{1}}}{\partial \xi_{1}^{p_{1}}} \cdots \frac{\partial^{p_{M}}}{\partial \xi^{p_{M}}} M\left\{\exp \left(-\frac{1}{2} \sum_{j=1}^{M} \xi_{i}^{2}\right)\right\} d \xi_{1} \cdots d \xi_{M} \\
& \text { where } \quad \mathscr{C}=\frac{(-1)^{p}(2 \pi)^{-\frac{M}{2}}}{\left\langle\mathscr{H}_{i}(\xi(\theta)\rangle_{L^{2}\left(\Theta^{(M)}, d P_{\xi}\right)}^{2}\right.} \exp \left(\overline{\mathbf{a}}_{0}\right)
\end{aligned}
$$

For the evaluation of the integral in Equation (A.4), we apply integration by parts with variable $\xi_{1}$ (whose order is $p_{1}$ ) and consider only a portion of the preceding integral as

$$
\begin{aligned}
\left(\mathbf{a}_{i}^{l}\right)_{1} & =\int_{-\infty}^{+\infty} \exp \left(\xi_{1} \tilde{\mathbf{a}}_{1}\right) \frac{\partial^{p_{1}}}{\partial \xi_{1}^{p_{1}}}\left\{\exp \left(-\frac{1}{2} \xi_{1}^{2}\right)\right\} d \xi_{1} \\
& =\left.\left(\exp \left(\xi_{1} \tilde{\mathbf{a}}_{1}\right) \frac{\partial^{p_{1-1}}}{\partial \xi_{1}^{p_{1}-1}}\left\{\exp \left(-\frac{1}{2} \xi_{1}^{2}\right)\right\}\right)\right|_{-\infty} ^{+\infty}-\int_{-\infty}^{+\infty} \frac{\partial}{\partial \xi_{1}}\left\{\exp \left(\xi_{1} \tilde{\mathbf{a}}_{1}\right)\right\} \frac{\partial^{p_{1}-1}}{\partial \xi_{1}^{p_{1}-1}}\left\{\exp \left(-\frac{1}{2} \xi_{1}^{2}\right)\right\} d \xi_{1}
\end{aligned}
$$

It is easy to see for the first term that

$$
\exp \left(\xi_{1} \tilde{\mathbf{a}}_{1}\right) \frac{\partial^{p_{1-1}}}{\partial \xi_{1}^{p_{1-1}}}\left\{\exp \left(-\frac{1}{2} \xi_{1}^{2}\right)\right\} \rightarrow 0 \quad \text { as } \quad \xi_{1} \rightarrow+\infty \quad \text { or, } \quad \xi_{1} \rightarrow-\infty
$$

because the exponential function is $C^{\infty}$ continuous and all the terms appearing in its successive derivatives would involve $\exp \left(-\frac{1}{2} \xi_{1}^{2}\right)$. Hence, the terms tend to 0 identically as $\xi_{1} \rightarrow-\infty,+\infty$. Thus, applying this procedure of integration by parts $p_{1}$ times and putting the leading terms to 0 (as in Equation (A.5)), we have

$$
\begin{aligned}
\left(\mathbf{a}_{i}^{l}\right)_{1} & =(-1)^{p_{1}} \int_{-\infty}^{+\infty} \frac{\partial^{p_{1}}}{\partial \xi_{1}^{p_{1}}}\left\{\exp \left(\xi_{1} \tilde{\mathbf{a}}_{1}\right)\right\}\left\{\exp \left(-\frac{1}{2} \xi_{1}^{2}\right)\right\} d \xi_{1} \\
& =(-1)^{p_{1}} \tilde{\mathbf{a}}_{1}^{p_{1}} \int_{-\infty}^{+\infty} \exp \left(-\frac{1}{2} \xi_{1}^{2}+\xi_{1} \tilde{\mathbf{a}}_{1}\right) d \xi_{1}
\end{aligned}
$$

The Gaussian function integral identity gives $\int_{-\infty}^{+\infty} a_{0} \exp \left(-a_{1} x^{2}+a_{2} x+a_{3}\right)=$ $a_{0} \sqrt{\pi / a_{1}} \exp \left(\frac{a_{2}^{2}}{4 a_{1}}+a_{3}\right)$ for $a_{1}>0$ following which we write Equation (A.6) as

$$
\left(\mathbf{a}_{i}^{l}\right)_{1}=(-1)^{p_{1}} \tilde{\mathbf{a}}_{1}^{p_{1}} \sqrt{2 \pi} \exp \left(\frac{1}{2} \tilde{\mathbf{a}}_{1}^{2}\right)
$$


The preceding equation can be combined with Equation (A.4) to obtain

$$
\mathbf{a}_{i}^{l}=\mathscr{C} \int_{-\infty}^{+\infty} \cdots \int_{-\infty}^{+\infty}\left(\mathbf{a}_{i}^{l}\right)_{1} \prod_{i=2}^{M} \exp \left(\xi_{i} \tilde{\mathbf{a}}_{i}\right) \frac{\partial^{p_{2}}}{\partial \xi_{2}^{p_{2}}} \cdots \frac{\partial^{p_{M}}}{\partial \xi_{M}^{p_{M}}}\left\{\exp \left(-\frac{1}{2} \sum_{j=2}^{M} \xi_{i}^{2}\right)\right\} d \xi_{2} \cdots d \xi_{M}
$$

Applying the same procedure as in Equations (A.5)-(A.7) to the successive random variables from $\xi_{2}$ to $\xi_{M}$, we would have the integral evaluated as

$$
\begin{aligned}
\mathbf{a}_{i}^{l}= & \mathscr{C}\left(\mathbf{a}_{i}^{l}\right)_{1} \cdots\left(\mathbf{a}_{M}^{l}\right)_{M} \\
\text { or } \quad \mathbf{a}_{i}^{l}= & \frac{(-1)^{p}(2 \pi)^{-\frac{M}{2}}}{\left\langle\mathscr{H}_{i}(\xi(\theta)\rangle_{L^{2}\left(\Theta^{(M)}, d P_{\xi}\right)}^{2}\right.} \exp \left(\overline{\mathbf{a}}_{0}\right)\left((-1)^{p_{1}} \tilde{\mathbf{a}}_{1}^{p_{1}} \sqrt{2 \pi} \exp \left(\frac{1}{2} \tilde{\mathbf{a}}_{1}^{2}\right)\right) \\
& \cdots\left((-1)^{p_{M}} \tilde{\mathbf{a}}_{M}^{p_{M}} \sqrt{2 \pi} \exp \left(\frac{1}{2} \tilde{\mathbf{a}}_{M}^{2}\right)\right) \\
= & \frac{(-1)^{p}(2 \pi)^{-\frac{M}{2}}}{\left\langle\mathscr{H}_{i}(\xi(\theta)\rangle_{L^{2}\left(\Theta^{(M)}, d P_{\xi}\right)}^{2}\right.} \exp \left(\overline{\mathbf{a}}_{0}\right)\left((-1)^{p}(2 \pi)^{\frac{M}{2}} \tilde{\mathbf{a}}_{1}^{p_{1}} \cdots \tilde{\mathbf{a}}_{M}^{p_{M}} \prod_{i=1}^{M} \exp \left(\frac{1}{2} \tilde{\mathbf{a}}_{i}^{2}\right)\right)
\end{aligned}
$$

where in the last step, we have substituted back the value of $\mathscr{C}$ from Equation (A.4) and used the relation $p=p_{1}+\ldots+p_{M}$. Hence, the $i$ th coefficient of the lognormal field is expressed in closed form as

$$
\begin{aligned}
\mathbf{a}_{i}^{l} & =\exp \left(\overline{\mathbf{a}}_{0}\right) \prod_{i=1}^{m} \exp \left(\frac{1}{2} \tilde{\mathbf{a}}_{i}^{2}\right) \frac{\tilde{\mathbf{a}}_{1}^{p_{1}} \cdots \tilde{\mathbf{a}}_{M}^{p_{M}}}{\left\langle\mathscr{H}_{i}(\xi(\theta)\rangle_{L^{2}\left(\Theta^{(M)}, d P_{\xi}\right)}^{2}\right.} \\
& =\exp \left(\overline{\mathbf{a}}_{0}\right) \prod_{i=1}^{m} \exp \left(\frac{1}{2} \tilde{\mathbf{a}}_{i}^{2}\right) \frac{\mathscr{H}_{i}^{h d}\left(\tilde{\mathbf{a}}_{1}, \ldots, \tilde{\mathbf{a}}_{M}\right)}{\left\langle\mathscr{H}_{i}(\xi(\theta)\rangle_{L^{2}\left(\Theta^{(M)}, d P_{\xi}\right)}^{2}\right.}
\end{aligned}
$$

where $\mathscr{H}_{i}^{h d}\left(\tilde{\mathbf{a}}_{1}, \ldots, \tilde{\mathbf{a}}_{M}\right)=\tilde{\mathbf{a}}_{1}^{p_{1}} \cdots \tilde{\mathbf{a}}_{M}^{p_{M}}$

that is, $\mathscr{H}_{i}^{\text {hd }}\left(\tilde{\mathbf{a}}_{1}, \ldots, \tilde{\mathbf{a}}_{M}\right)$ denotes the term of highest degree (i.e., the term of degree $p_{1}, \ldots, p_{M}$ in random variables $\xi_{1}, \ldots, \xi_{M}$, respectively) of the $i$ th multivariate Hermite polynomial of degree $p$ associated with the $M$ Gaussian i.i.d. random variables.

\section{APPENDIX B: EXPRESSIONS FOR THE DETERMINANT OF A MATRIX SERIES WITH STOCHASTIC COEFFICIENTS}

We derive the determinant of a matrix series with scalar coefficients of the form

$$
\begin{aligned}
\mathbf{A} & =\sum_{i=1}^{m} \alpha_{i} \mathbf{A}_{i}, \quad \text { where } \quad \mathbf{A}, \mathbf{A}_{1}, \ldots, \mathbf{A}_{m} \in \mathbb{R} n n ; \quad \alpha_{i} \in \mathbb{R} \forall i \\
\text { with } \operatorname{det}|\mathbf{A}| & =\operatorname{det}\left|\sum_{i=1}^{m} \alpha_{i} \mathbf{A}_{i}\right|
\end{aligned}
$$

where $m$ can be greater than, equal to, or less than $n$, the dimension of the square matrices in the series. It is can be deduced from observation that the determinant in the preceding equation would 
be a degree $n$ homogeneous function of the coefficients $\alpha_{i}$. Let us define a set $\mathscr{M}$, which consists of all powers (from 1 to $n$ ) of the scalars $\alpha_{i}$, as

$$
\mathscr{M}=\left\{\alpha_{1}, \alpha_{1}^{2}, \ldots, \alpha_{1}^{n}, \ldots, \alpha_{m}, \alpha_{m}^{2}, \ldots, \alpha_{m}^{n}\right\}
$$

and $M$ is the set of all the elements derived from $\mathscr{M}$, which satisfies the following condition:

$$
M=\left\{\tilde{\alpha}_{i}: \tilde{\alpha}_{i}=\prod_{j=1}^{r} \alpha_{j}^{p_{j}} \text { with } \sum_{j=1}^{r} p_{j}=n, \text { for all } 1 \leqslant r \leqslant m\right\}
$$

and let $\mathscr{I}_{M}$ be the cardinality of this set. In other words, the elements of the set $M$ are such that the total sum of their powers is always equal to $n$. The determinant of $\mathbf{A}$ can be expressed as

$$
\operatorname{det}|\mathbf{A}|=\sum_{i \in \mathscr{I}_{M}} \tilde{\alpha}_{i}\left(\sum_{j \in \mathscr{I}_{n}} \operatorname{det}\left|\tilde{\mathbf{A}}_{j}\right|\right)
$$

where the $\tilde{\mathbf{A}}_{j}$ matrices are generated from combining the rows of the matrices $\mathbf{A}_{i}$ (from Equation (B.2)) associated with the scalar $\alpha_{i}$-s (in the coefficient $\tilde{\alpha}_{i}$ ) according to their powers in $\tilde{\alpha}_{i}$. For example, if the coefficient associated with the scalar term $\tilde{\alpha}_{i}=\alpha_{1}^{p_{1}} \ldots \alpha_{m}^{p_{m}}$ is sought, then we define

$$
\begin{aligned}
S & =\left\{x: x=\text { permutation of } \mathrm{p}_{1} \text { number of } \mathrm{j}_{1}, \mathrm{p}_{2} \text { number of } \mathrm{j}_{2}, \ldots, \mathrm{p}_{\mathrm{m}} \text { number of } \mathrm{j}_{\mathrm{m}}\right\} \\
c_{i} & =\sum_{j} \operatorname{det}\left|\tilde{\mathbf{A}}_{x}\right| \quad \forall x \in S
\end{aligned}
$$

where $\tilde{\mathbf{A}}_{x}$ denotes the matrix constructed from taking all permutations of $p_{1}$ rows taken from $\mathbf{A}_{j_{1}}$ matrix, $p_{2}$ rows taken from $\mathbf{A}_{j_{2}}$ matrix, and so on up to $p_{m}$ rows taken from $\mathbf{A}_{j_{m}}$ matrix. It should be noted that the dimension of $\tilde{\mathbf{A}}_{x}$ is $n \times n$ so that $p_{1}+\ldots+p_{m}=n$. To illustrate the point, we provide a numerical example of a matrix series of $3 \times 3$ matrix $\tilde{\mathbf{A}}$ expressed as $\tilde{\mathbf{A}}=\alpha_{1} \tilde{\mathbf{A}}_{1}+\alpha_{2} \tilde{\mathbf{A}}_{2}+\alpha_{3} \tilde{\mathbf{A}}_{3}$. The determinant of $\tilde{\mathbf{A}}$ is expressed with polynomials of $\alpha_{i}, i=1,2,3$, as

$$
\begin{aligned}
\operatorname{det}|\tilde{\mathbf{A}}|= & \alpha_{1}^{3} \operatorname{det}\left|\tilde{\mathbf{A}}_{j_{1} j_{1} j_{1}}\right|+\alpha_{2}^{3} \operatorname{det}\left|\tilde{\mathbf{A}}_{j_{2} j_{2} j_{2}}\right|+\alpha_{3}^{3} \operatorname{det}\left|\tilde{\mathbf{A}}_{j_{3} j_{3} j_{3}}\right|+\alpha_{1}^{2} \alpha_{2} \operatorname{det}\left|\tilde{\mathbf{A}}_{j_{1} j_{1} j_{2}}\right|+\alpha_{1}^{2} \alpha_{3} \operatorname{det}\left|\tilde{\mathbf{A}}_{j_{1} j_{1} j_{3}}\right| \\
& +\alpha_{2}^{2} \alpha_{1} \operatorname{det}\left|\tilde{\mathbf{A}}_{j_{2} j_{2} j_{1}}\right|+\alpha_{2}^{2} \alpha_{3} \operatorname{det}\left|\tilde{\mathbf{A}}_{j_{2} j_{2} j_{3}}\right|+\alpha_{3}^{2} \alpha_{1} \operatorname{det}\left|\tilde{\mathbf{A}}_{j_{3} j_{3} j_{1}}\right|+\alpha_{3}^{2} \alpha_{2} \operatorname{det}\left|\tilde{\mathbf{A}}_{j_{3} j_{3} j_{2}}\right| \\
& +\alpha_{1} \alpha_{2} \alpha_{3} \operatorname{det}\left|\tilde{\mathbf{A}}_{j_{1} j_{2} j_{3}}\right|
\end{aligned}
$$

where det $\left|\tilde{\mathbf{A}}_{j_{1} j_{1} j_{1}}\right|=\operatorname{det}\left|\mathbf{A}_{1}\right|$;

$$
\operatorname{det}\left|\tilde{\mathbf{A}}_{j_{1} j_{1} j_{2}}\right|=\left[\operatorname{det}\left|\begin{array}{l}
-\left(\mathbf{A}_{1}\right)_{1}- \\
-\left(\mathbf{A}_{1}\right)_{2}- \\
-\left(\mathbf{A}_{2}\right)_{3}-
\end{array}\right|+\operatorname{det}\left|\begin{array}{l}
-\left(\mathbf{A}_{1}\right)_{1}- \\
-\left(\mathbf{A}_{2}\right)_{2}- \\
-\left(\mathbf{A}_{1}\right)_{3}-
\end{array}\right|+\operatorname{det}\left|\begin{array}{l}
-\left(\mathbf{A}_{2}\right)_{1}- \\
-\left(\mathbf{A}_{1}\right)_{2}- \\
-\left(\mathbf{A}_{1}\right)_{3}-
\end{array}\right|\right]
$$

and $\operatorname{det}\left|\tilde{\mathbf{A}}_{j_{1} j_{2} j_{3}}\right|=\left[\operatorname{det}\left|\begin{array}{l}-\left(\mathbf{A}_{1}\right)_{1}- \\ -\left(\mathbf{A}_{2}\right)_{2}- \\ -\left(\mathbf{A}_{3}\right)_{3}-\end{array}\right|+\operatorname{det}\left|\begin{array}{l}-\left(\mathbf{A}_{1}\right)_{1}- \\ -\left(\mathbf{A}_{3}\right)_{2}- \\ -\left(\mathbf{A}_{2}\right)_{3}-\end{array}\right|+\operatorname{det}\left|\begin{array}{l}-\left(\mathbf{A}_{2}\right)_{1}- \\ -\left(\mathbf{A}_{1}\right)_{2}- \\ -\left(\mathbf{A}_{3}\right)_{3}-\end{array}\right|+\operatorname{det}\left|\begin{array}{l}-\left(\mathbf{A}_{2}\right)_{1}- \\ -\left(\mathbf{A}_{3}\right)_{2}- \\ -\left(\mathbf{A}_{1}\right)_{3}-\end{array}\right|\right.$

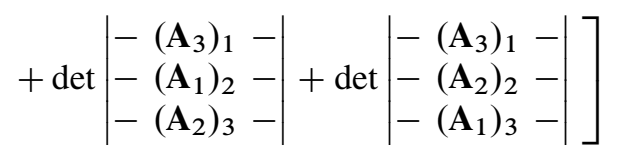

In the preceding expressions, $\left(\mathbf{A}_{i}\right)_{j}$ denotes the $j$ th row of the $\mathbf{A}_{i}$ matrix from the series representation in Equation (B.1). Hence, while forming the combination of matrix rows to evaluate the 
determinant associated with a particular scalar term (say $\prod_{i} \alpha_{i}^{p_{i}}$ ), the number of rows chosen from each coefficient matrix (say $\mathbf{A}_{i}$ ) is equal to the power of the scalar term (i.e., $p_{i}$ ) present in that coefficient. The chosen columns are permuted to give all possible combination of the coefficient matrices. Because $\sum_{i} p_{i}=n$, the resulting individual matrices would always be square matrices.

\section{ACKNOWLEDGEMENTS}

A. K. acknowledges the support of Swansea University through the award of the Zienkiewicz Scholarship. S. A. gratefully acknowledges the support of The Royal Society of London through the Wolfson Research Merit Award. M. I. F. acknowledges funding from the European Research Council through grant no. 247045 entitled 'Optimisation of Multi-scale Structures with Applications to Morphing Aircraft'.

\section{REFERENCES}

1. Nouy A. Recent developments in spectral stochastic methods for the numerical solution of stochastic partial differential equations. Archives of Computational Methods in Engineering 2009; 16:251-285.

2. Stefanou G. The stochastic finite element method: past, present and future. Computer Methods in Applied Mechanics and Engineering 2009; 198(9-12):1031-1051.

3. Kundu A, Adhikari S. Transient Response of Structural Dynamic Systems with Parametric Uncertainty. Journal Engineering Mechanics 2013; 140(2):315-331.

4. Babuška I, Chleboun J. Effects of uncertainties in the domain on the solution of Neumann boundary value problems in two spatial dimensions. Mathematics of Computation 2002; 71(240):1339-1370.

5. Babuška I, Chleboun J. Effects of uncertainties in the domain on the solution of Dirichlet boundary value problems. Numerische Mathematik 2003; 93(4):583-610.

6. Ganapathysubramanian B, Zabaras N. Sparse grid collocation schemes for stochastic natural convection problems. Journal of Computational Physics 2007; 225(1):652-685.

7. Zayernouri M, Park SW, Tartakovsky DM, Karniadakis GE. Stochastic smoothed profile method for modeling random roughness in flow problems. Computer Methods in Applied Mechanics and Engineering 2013; 263(0):99-112.

8. Tartakovsky DM, Xiu D. Stochastic analysis of transport in tubes with rough walls. Journal of Computational Physics 2006; 217(1):248-259.

9. Xiu D, Shen J. An efficient spectral method for acoustic scattering from rough surfaces. Communications in Computational Physics 2007; 2(1):54-72.

10. Nicholls DP, Shen J. A stable, high-order method for two-dimensional bounded-obstacle scattering. SIAM Journal on Scientific Computing 2006; 28(4):1398-1419.

11. Blyth MG, Pozrikidis C. Heat conduction across irregular and fractal-like surfaces. International Journal of Heat and Mass Transfer 2003; 46(8):1329-1339.

12. Xiu D, Tartakovsky DM. Numerical methods for differential equations in random domains. SIAM Journal on Scientific Computing 2006; 28(3):1167-1185.

13. Thompson JF, Warsi ZU, Mastin CW. Numerical Grid Generation: Foundations and Applications, Vol. 38. North-Holland: Amsterdam, 1985.

14. Adler RJ, Taylor JE. Random Fields and Geometry, Vol. 115. Springer: New York, USA, 2007.

15. Oksendal B. Stochastic Differential Equations: An Introduction with Applications. Springer: New York, 1995.

16. Ogorodnikov V, Prigarin S. Numerical Modelling of Random Processes and Fields: Algorithms and Applications. VSP: Utrecht, 1996.

17. Grigoriu M. Non-Gaussian models for stochastic mechanics. Probabilistic Engineering Mechanics 2000; 15(1): $15-23$.

18. Kolovos A, Christakos G, Hristopulos D, Serre M. Methods for generating non-separable spatiotemporal covariance models with potential environmental applications. Advances in Water Resources 2004; 27(8):815-830.

19. Christakos G. Random Field Models in Earth Sciences. Academic Press: San Diego, CA, 1992.

20. Liu WK, Belytschko T, Mani A. Random field finite-elements. International Journal for Numerical Methods in Engineering 1986; 23(10):1831-1845.

21. Li CC, Kiureghian AD. Optimal discretization of random fields. Journal of Engineering Mechanics 1993; 119(6):1136-1154.

22. Vanmarcke EH. Random Fields. MIT Press: Cambridge Mass., 1983.

23. Xiu D, Karniadakis GE. Modeling uncertainty in flow simulations via generalized polynomial chaos. Journal of Computational Physics 2003; 187(1):137-167.

24. Porter D, Stirling D. Integral Equations: A Practical Treatment, from Spectral Theory to Applications. Cambridge University Press: Cambridge, UK, 1990.

25. Ghanem R. The nonlinear Gaussian spectrum of log-normal stochastic processes and variables. Journal of Applied Mechanics-ASME 1989; 66:964-973.

26. Rahman S, Xu H. A meshless method for computational stochastic mechanics. International Journal for Computational Methods in Engineering Science and Mechanics 2005; 6(1):41-58. 
27. Allaix DL, Carbone VI. Karhunen-Loève decomposition of random fields based on a hierarchical matrix approach. International Journal for Numerical Methods in Engineering 2013; 94(11):1015-1036.

28. Graham IG, Kuo FY, Nuyens D, Scheichl R, Sloan IH. Quasi-Monte Carlo methods for elliptic PDEs with random coefficients and applications. Journal of Computational Physics 2011; 230(10):3668-3694.

29. Adler RJ. The Geometry of Random Fields. Wiley: New York, USA, 1981.

30. Ghanem R, Spanos PD. Stochastic Finite Elements: A Spectral Approach. Springer-Verlag: New York, USA, 1991.

31. Babuška I, Tempone R, Zouraris GE. Solving elliptic boundary value problems with uncertain coefficients by the finite element method: the stochastic formulation. Computer Methods in Applied Mechanics and Engineering 2005; 194(12-16):1251-1294.

32. Deb MK, Babuška IM, Oden JT. Solution of s using Galerkin finite element techniques. Computer Methods in Applied Mechanics and Engineering 2001; 190(48):6359-6372.

33. Babuška I, Tempone R, Zouraris GE. Galerkin finite element approximations of stochastic elliptic partial differential equations. Siam Journal on Numerical Analysis 2004; 42(2):800-825.

34. Pettit C, Beran P. Spectral and multiresolution wiener expansions of oscillatory stochastic processes. Journal of Sound and Vibration 2006; 294(45):752-779.

35. Keese A. A review of recent developments in the numerical solution of s (stochastic finite elements). Report No. Informatikbericht 2003, 2003; 6.

36. Rahman S. A polynomial dimensional decomposition for stochastic computing. International Journal for Numerical Methods in Engineering 2008; 76(13):2091-2116.

37. Rahman S, Yadav V. Orthogonal polynomial expansions for solving random eigenvalue problems. International Journal for Uncertainty Quantification 2011; 1(2):163-187.

38. Nouy A. Generalized spectral decomposition method for solving stochastic finite element equations: invariant subspace problem and dedicated algorithms. Computer Methods in Applied Mechanics and Engineering 2008; 197(51-52):4718-4736.

39. Schuëller GI. Computational stochastic mechanics—recent advances. Computers and Structures 2001; 79: 2225-2234.

40. Taylor G. A model for the boundary condition of a porous material. Part 1. Journal of Fluid Mechanics 1971; 49(02):319-326.

41. Richardson S. A model for the boundary condition of a porous material. part 2. Journal of Fluid Mechanics 1971; 49(2):327-336.

42. Blyth M, Pozrikidis C. Heat conduction across irregular and fractal-like surfaces. International Journal of Heat and Mass Transfer 2003; 46(8):1329-1339.

43. Nouy A, Clément A, Schoefs F, Moës N. An extended stochastic finite element method for solving stochastic partial differential equations on random domains. Computer Methods in Applied Mechanics and Engineering 2008; 197(51-52):4663-4682.

44. Nouy A, Clément A. Extended stochastic finite element method for the numerical simulation of heterogeneous materials with random material interfaces. International Journal for Numerical Methods in Engineering 2010; 83(10): 1312-1344.

45. Mohan PS, Nair PB, Keane AJ. Stochastic projection schemes for deterministic linear elliptic partial differential equations on random domains. International Journal for Numerical Methods in Engineering 2011; 85(7):874-895.

46. Reddy JN. An Introduction to the Finite Element Method. McGraw-Hill: New York, 1993.

47. Kundu A, DiazDelaO F, Adhikari S, Friswell M. A hybrid spectral and metamodeling approach for the stochastic finite element analysis of structural dynamic systems. Computer Methods in Applied Mechanics and Engineering 2014; 270(0):201-219.

48. Zienkiewicz OC, Taylor RL. The Finite Element Method (4th edn). McGraw-Hill: London, 1991.

49. Li R, Ghanem R. Adaptive polynomial chaos expansions applied to statistics of extremes in nonlinear random vibration. Probabilistic Engineering Mechanics 1998; 13(2):125-136.

50. Blatman G, Sudret B. An adaptive algorithm to build up sparse polynomial chaos expansions for stochastic finite element analysis. Probabilistic Engineering Mechanics 2010; 25(2):183-197.

51. Yadav V, Rahman S. Adaptive-sparse polynomial dimensional decomposition methods for high-dimensional stochastic computing. Computer Methods in Applied Mechanics and Engineering 2014; 274(0):56-83.

52. Sakamoto S, Ghanem R. Simulation of multi-dimensional non-Gaussian non-stationary random fields. Probabilistic Engineering Mechanics 4 2002; 17(2):167-176. 